\title{
Microglial MorphOMICs unravel region- and sex-dependent morphological phenotypes from postnatal development to degeneration
}

\section{Authors}

Gloria Colombo $^{1 *}$, Ryan John A. Cubero ${ }^{1 *}$, Lida Kanari ${ }^{2}$, Alessandro Venturino ${ }^{1}$, Rouven Schulz $^{1}$, Martina Scolamiero ${ }^{3}$, Jens Agerberg ${ }^{3}$, Hansruedi Mathys ${ }^{4,5,6}$, Li-Huei Tsai ${ }^{4,5,6}$, Wojciech Chachólski ${ }^{3}$, Kathryn Hess $^{7}$, Sandra Siegert ${ }^{1 \dagger}$

\section{Author affiliations}

${ }^{1}$ Institute of Science and Technology (IST) Austria, Am Campus 1, 3400 Klosterneuburg, Austria.

${ }^{2}$ Blue Brain Project, École polytechnique fédérale de Lausanne (EPFL),

Campus Biotech, 1202 Geneva, Switzerland.

${ }^{3}$ KTH Royal Institute of Technology, Stockholm, Sweden.

${ }^{4}$ Picower Institute for Learning and Memory, Massachusetts Institute of Technology, Cambridge, MA, USA.

${ }^{5}$ Department of Brain and Cognitive Sciences, Massachusetts Institute of Technology, Cambridge, MA, USA.

${ }^{6}$ Broad Institute of Harvard and MIT, Cambridge, MA, USA.

${ }^{7}$ Laboratory for topology and neuroscience, Brain Mind Institute, École polytechnique fédérale de Lausanne (EPFL), 1015 Lausanne, Switzerland.

${ }^{*}$ Contributed equally

†Corresponding author: Sandra Siegert, sandra.siegert@ist.ac.at

\section{Keywords}

Microglia, morphology, phenotypic spectrum, brain region, sexual dimorphism, spatial heterogeneity, development, Alzheimer's degeneration, topology, 5xFAD, CK-p25 


\begin{abstract}
$\underline{\text { Abstract }}$
Microglia contribute to tissue homeostasis in physiological conditions with environmental cues influencing their ever-changing morphology. Strategies to identify these changes usually involve user-selected morphometric features, which, however, have proved ineffective in establishing a spectrum of context-dependent morphological phenotypes. Here, we have developed MorphOMICs, a topological data analysis approach to overcome feature-selectionbased biases and biological variability. We extracted a spatially heterogeneous and sexuallydimorphic morphological phenotype for seven adult brain regions, with ovariectomized females forming their own distinct cluster. This sex-specific phenotype declines with maturation but increases over the disease trajectories in two neurodegeneration models, 5xFAD and CK-p25. Females show an earlier morphological shift in the immediatelyaffected brain regions. Finally, we demonstrate that both the primary- and the short terminal processes provide distinct insights to morphological phenotypes. MorphOMICs maps microglial morphology into a spectrum of cue-dependent phenotypes in a minimally-biased and semi-automatic way.
\end{abstract}




\section{Introduction}

Morphological characterization of neuronal shapes has provided important insights into the diversity of cell types related to their genetic and functional features (Gouwens et al., 2019; Jiang et al., 2015; Moschovakis et al., 1988). Numerous studies have tried to apply a similar morphological analysis on microglia (Adeluyi et al., 2019; Heindl et al., 2018; Kongsui et al., 2014; Morrison and Filosa, 2013; Morrison et al., 2017; Young and Morrison, 2018).

Although they indicated a microglia heterogeneity (Bachstetter et al., 2015; Lawson et al., 1990; Stratoulias et al., 2019; Tan et al., 2019), no study has found a way to capture contextspecific responses during development and degeneration. Moreover, the majority of these analysis rely on restricted microglia sample sizes underestimating their full morphological phenotypical spectrum. Detecting subtle changes in the microglial morphology along the spectrum would offer an early readout of their immediate responses to local environmental cues (Lawson et al., 1990), as microglia are sensitive to changes in neuronal activity (González-Scarano and Baltuch, 1999; Greter and Merad, 2013; Paolicelli et al., 2011; PontLezica et al., 2011; Venturino et al., 2021).

Microglia morphological phenotype is commonly determined with user-selected features from a three-dimensional (3D) reconstructed branching tree: these features can include total process length, branch number, or number of terminal points. These scalar morphometric descriptors are then statistically compared across conditions. The drawback of this approach is the number and the type of selected features, which biases the biological readout: while too few selected features underrepresent a phenotypic difference, too many cause overfitting and introduce noise (Carlsson, 2009). Moreover, in contrast to neuronal morphological trees that are static on the gross structure, microglia processes are highly dynamic (Davalos et al., 2008; Nimmerjahn et al., 2005; Tremblay et al., 2011; Wu et al., 2015) as they constantly survey their local environment (Davalos et al., 2008). This introduces considerable intrinsic variability within the traced microglia population of a defined condition as well as the risk of selection bias to the extreme phenotypes. Establishing a reliable brain-region-specific morphological phenotype is critical for characterizing baseline morphology and tracking changes as deviations from the baseline.

To capture morphological phenotypes, complex morphological trees must be simplified with minimal information loss, retaining as many features as possible. Algebraic topology 
provides new strategies for solving this problem, as it focuses on the shape properties of geometric objects without the need of morphometrics (Kanari et al., 2017; Li et al., 2017). In particular, the topological morphology descriptor (TMD), which assigns a barcode to any three-dimensional tree, has been successfully applied for classification of cortical neuron morphologies (Kanari et al., 2017, 2020). When we first applied the TMD to 10,000 traced microglia across the rostro-caudal axis of seven selected adult brain regions, the data indicated a regional phenotype, but the diversity of the individual microglia obscured any well-defined separation. We therefore developed our MorphOMICs pipeline to overcome the major limitations of feature-selection-based analysis and biological data variability. MorphOMICs combines TMD with bootstrapping, dimensionality reduction, and data visualization techniques, enabling minimally-biased identification of the baseline phenotype. We found that adult microglia are brain-region-specific, with sex as a confounding factor. Remarkably, microglia from ovariectomized females did not match the corresponding brain regions of males nor females, underscoring the impact of sex on the morphological phenotypic spectrum. This was further strengthened when we analyzed postnatal development and degeneration. We found an early microglial sexual dimorphism, which gradually declined towards adulthood. In contrast, the sex-specific phenotype diverged during disease progression in two distinct Alzheimer's disease models, 5xFAD and CK-p25, where females differ in their context-dependent response from males. When we aligned the trajectories of development and degeneration, we observed a phenotypic spectrum supporting that microglia morphology is context- and sex-dependent. Finally, we found that both short and long persistence bars are required to detect subtle changes within microglia morphology. MorphOMICs provides a minimally-biased, semi-automatic strategy for mapping a spectrum of microglia phenotypes across conditions.

\section{$\underline{\text { Results }}$}

\section{MorphOMICs uncovers adult microglial heterogeneity beyond intrinsic variability}

To address how morphological phenotypes differ between microglia across brain regions, we immunostained the adult C57BL/6J mouse brain with the allograft inflammatory factor 1 (Iba1/Aif1) (Ito et al., 1998) for both sexes with at least biological triplicates. Then, we traced 9,997 cells and generated three-dimensional (3D) microglial skeletons from seven brain regions chosen to span the rostro-caudal axis with a preference for regions that are known to be affected in Alzheimer disease (Braak et al., 1989; Brar et al., 2009; Burns et al., 2005; 
DeKosky and Scheff, 1990; Gosche et al., 2002; Jacobs et al., 2018; Kazee et al., 1995; Kovács et al., 1999; Larner, 1997; Lehtovirta et al., 1995; Leuba et al., 2008; Ohm and Braak, 1987, 1989; Rao et al., 2012; Rombaux et al., 2010; Sinha et al., 1993; Stephen et al., 2010; Thompson et al., 2004; Wiesman et al., 2021; Zarow et al., 2003): the olfactory bulb (OB $\mathrm{Bg}_{\mathrm{mg}}$ ), frontal cortex $\left(\mathrm{FC}_{\mathrm{mg}}\right)$, dentate gyrus of the hippocampus $\left(\mathrm{DG}_{\mathrm{mg}}\right)$, primary somatosensory cortex $\left(\mathrm{S} 1_{\mathrm{mg}}\right)$, substantia nigra $\left(\mathrm{SN}_{\mathrm{mg}}\right)$, cochlear nucleus $\left(\mathrm{CN}_{\mathrm{mg}}\right)$, and cerebellum $\left(\mathrm{CB}_{\mathrm{mg}}\right.$, Fig. 1A). When we utilized morphometrics that are commonly used in the field of microglial morphology (Kozlowski and Weimer, 2012; Morrison and Filosa, 2013; Zusso et al., 2012), we found non-significant differences across these brain regions with the exception of (CB and $\mathrm{CN})_{\mathrm{mg}}$ (Supp. Fig. 1A). We therefore applied the topological morphology descriptor (TMD) (Kanari et al., 2017; Li et al., 2017) for which each 3D skeleton was represented as a rooted tree with the microglial soma, processes, branching points, and process terminals (Fig. 1B, i). The TMD converts the tree as a persistence barcode, where each bar represents the persistent process lifetime in terms of the radial distance from the soma (Carlsson, 2009; Kanari et al., 2017). Every bar is then collapsed into a single point in the persistence diagram summarizing the process's lifetime, which is then converted into a persistence image using Gaussian kernels (Adams et al., 2017) (Fig. 1B, ii, iii). The branching complexity is spatially represented by process length proportional to the distance from the diagonal (Fig. 1B, iv). To quantify the differences between microglial morphologies across brain regions, we computed the pairwise TMD distance between the average persistence images (Kanari et al., 2017). We identified clusters with $(\mathrm{OB}, \mathrm{FC}, \mathrm{SN})_{\mathrm{mg}}$ and $(\mathrm{S} 1, \mathrm{DG})_{\mathrm{mg}}$, whereas $\mathrm{CN}_{\mathrm{mg}}$ and $\mathrm{CB}_{\mathrm{mg}}$ were segregated (Supp. Fig. 1B). The average persistence images also did not differ strongly (Fig. 1C). When we looked at the individual persistence images, we found a wide variance that makes it challenging to distinguish regional phenotypes (Supp. Fig. 1C). We note that this dispersion is not driven by an animal-based batch effect (Supp. Fig. 1D).

To overcome this intrinsic variability within a microglial population, we developed MorphOMICs, which combines TMD with random subsampling of persistence images, dimensionality reduction, and data visualization strategies. Bootstrapping randomly draws, without replacement, a user-defined number of unique persistence images $(\mathrm{x})$ from a microglial population pool (n) and iteratively generates bootstrapped persistence images (Fig. 1D). To display these bootstrapped persistence images for each brain region, we applied the nonlinear dimensionality reduction technique UMAP (Uniform Manifold Approximation and Projection, Fig. 1E), which converts the high-dimensional persistence images into a reduced 
2D representation while preserving their global structure (Mcinnes et al., 2020). While local distances are preserved in UMAP compared to t-SNE (Van Der Maaten and Hinton, 2008), the point's actual position in the reduced space is irrelevant. After controlling for the bootstrapped to microglial population pool size ratio (Supp. Fig. 2A-E, Supplementary Text), we applied MorphOMICs to our data. The UMAP plot exhibited a spatial separation similar to that of the distance matrix in Supp. Fig. 1B, with $\mathrm{CB}_{\mathrm{mg}}$ separated from the other brain regions and $(\mathrm{OB}, \mathrm{FC}, \mathrm{SN})_{\mathrm{mg}}$ occupying a well-defined area in the UMAP space (Fig. 1F). Interestingly, MorphOMICs revealed that $\mathrm{OB}_{\mathrm{mg}}$ and $\mathrm{FC}_{\mathrm{mg}}$ are intermingled, while $\mathrm{DG}_{\mathrm{mg}}$ and $\mathrm{S} 1 \mathrm{mg}$ formed distinct clusters. Importantly, these cluster segregations were stable even if we changed UMAP's hyperparameters (Supp. Fig. 2F) or when we applied t-SNE visualization instead (Supp. Fig. 2G). More complex morphological relationships between brain regions can exist as exhibited by $\mathrm{CN}_{\mathrm{mg}}$. When we represented the persistence barcodes with stable ranks instead of persistence images (Agerberg et al., 2021; Riihimäki and Chacholski, 2018), the region-specific phenotypes were maintained (Supp. Fig. 2H). Furthermore, stable ranks allowed us to discriminate microglia in to different brain regions with a classification accuracy that reflects the separation between brain regions in the UMAP space (Supp. Fig. 3A). An alternative morphological simplification that is commonly performed in the literature is Sholl analysis, which calculates the number of processes that intersect concentric spheres centered on the soma with a user-defined radius (Sholl, 1953). When we applied Sholl analysis we could not recapitulate entirely the spatial segregation captured by MorphOMICs (Supp. Fig. 3B). Even if we applied bootstrapping to Shollanalysis, we could only dissect the regional heterogeneity for $\mathrm{CB}_{\mathrm{mg}}$ and $\mathrm{CN}_{\mathrm{mg}}$ (Supp. Fig. 3C). Interestingly, the clusters become less distinct by increasing the Sholl step size radius confirming the superiority of MorphOMICs. Overall, these data indicate that adult brain regions have well-defined microglia morphological phenotypes, which is uncovered by MorphOMICs.

\section{The microglial phenotype displays sexual dimorphism in a brain-region-dependent manner}

The extent of microglial sexual dimorphism across brain regions is only partially understood (Han et al., 2021; Lenz et al., 2013; Nelson et al., 2017). We applied MorphOMICs to all brain regions, and compared males and females within the UMAP space (Fig. 2A-B). Each brain region occupied a unique cluster in the plot, where $\mathrm{CB}_{\mathrm{mg}}$ and $\mathrm{CN}_{\mathrm{mg}}$ were most 
divergent. Strikingly, most brain regions separated female and male microglia, with $\mathrm{CB}_{\mathrm{mg}}$, $\mathrm{CN}_{\mathrm{mg}}, \mathrm{SN}_{\mathrm{mg}}, \mathrm{S}_{\mathrm{mg}}$, and $\mathrm{OB}_{\mathrm{mg}}$ forming close but spatially separated clusters. In contrast,

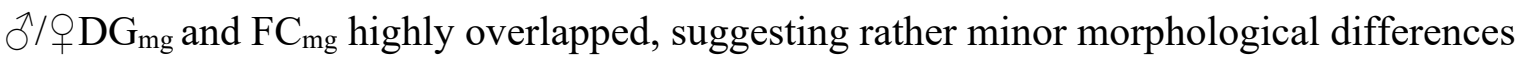
between the sexes. Interestingly, compared to Supp. Fig. 1B the (FC, OB $)_{\mathrm{mg}}$ cluster broke up: ${ }^{\lambda} \mathrm{FC}_{\mathrm{mg}}$ and $\delta^{\lambda} \mathrm{OB}_{\mathrm{mg}}$ formed spatially separated clusters, whereas $q(\mathrm{FC}, \mathrm{OB})_{\mathrm{mg}}$ were intermingled.

The morphological differences could depend on the microglia distribution; therefore, we determined the microglia density for each brain region and sex. We found that the density was the lowest for both sexes in $\mathrm{CB}_{\mathrm{mg}}$ (Supp. Fig. 4A) and only $\mathrm{CN}_{\mathrm{mg}}$ and $\mathrm{OB}_{\mathrm{mg}}$ showed a significant sexual dimorphism. This is reflected within the UMAP space, where both brain regions showed the strongest separation (Fig. 2B). To determine whether this sex-specific intermingling is hormone-dependent, we traced microglia from adult females that we ovariectomized at P20 ( ${ }_{+o v}$, Supp. Fig. 4B) before they start the estrous cycle and enter puberty (Caligioni, 2009). We found that the ${ }_{+}{ }_{\mathrm{ov}} \mathrm{FC}_{\mathrm{mg}}$ cluster no longer intermingled with ${ }_{\text {ov }} \mathrm{OB}_{\mathrm{mg}}$ in ovariectomized females but instead fused with ${ }_{\text {ovv }} \mathrm{SN}_{\mathrm{mg}}$ (Fig. 2C). This is surprising, as in non-ovariectomized mice, ${ }_{\rightarrow} \mathrm{SN}_{\mathrm{mg}}$ was close to but distinct from the intermingled $q(\mathrm{FC}, \mathrm{OB})_{\mathrm{mg}}$. When we compared non-ovariectomized to ovariectomized females, we found that in the UMAP space ovariectomized females formed distinct clusters, spatially separated from their non-ovariectomized counterparts (Fig. 2B-C). These results demonstrate the existence of brain-region-specific, sexually dimorphic phenotype, and that interfering with estrogen production before puberty affects microglial heterogeneity.

\section{Sexual dimorphism affects microglial morphology during development}

Microglia originate in the yolk sac and infiltrate the nervous system early during embryonic development (Ginhoux et al., 2013). After microglia occupy a brain region, their morphology gradually becomes more branched during postnatal neuronal circuit refinement (Fig. 3A) (Ben-Ari, 2002; Ginhoux et al., 2010; Kroon et al., 2019; Perez-Pouchoulen et al., 2015; Ruusuvuori et al., 2004; Valeeva et al., 2016; Wong et al., 2005; Yang et al., 2014). To determine whether microglial heterogeneity and dimorphic phenotype already exist within the first postnatal weeks and before the onset of puberty, we sampled microglia from all seven brain regions at postnatal days 7, 15, and 22 (Supp. Fig. 5A-B) and highlighted either each brain region or the developmental time point in the UMAP plots (Fig. 3B-C, respectively). In all seven brain regions, no postnatal time points overlapped with the adult microglia (Fig. 3B), reflecting 
their morphological spectrum during development. When we analyzed each time point individually, we found that at P7, all brain regions are distinct but occupy the same cluster, which shifted to a different cluster at P15 (Fig. 3C). Interestingly, $\mathrm{CN}_{\mathrm{mg}}$ and $\mathrm{DG}_{\mathrm{mg}}$ segregated and remained distinct from the other brain regions at $\mathrm{P} 15$ and $\mathrm{P} 22$, with $\mathrm{CB}_{\mathrm{mg}}$ joining them at P22. Between P22 and adulthood, the clusters diverged to their adult microglial heterogeneity.

Next, we investigated whether sexual dimorphism affects microglial phenotypic spectrum during development. To do this, we applied the MorphOMICs pipeline to males and females separately (Fig. 3D, Supp. Fig. 5C). Surprisingly, we found that the clusters shown in Figure 3C split, leading to well-defined male and female clusters for each brain region at P7. With brain maturation, $P / \widehat{O}$ clusters in $\mathrm{S}_{1 \mathrm{mg}}, \mathrm{DG}_{\mathrm{mg}}$, and $\mathrm{FC}_{\mathrm{mg}}$ converged, while those in $\mathrm{OB}_{\mathrm{mg}}$, $\mathrm{SN}_{\mathrm{mg}}, \mathrm{CB}_{\mathrm{mg}}$, and $\mathrm{CN}_{\mathrm{mg}}$ remained distinct. To follow this sexual dimorphism along the developmental trajectory, we ordered the bootstrapped persistence images with the Palantir algorithm (Setty et al., 2019), which uses principles from graph theory and Markov processes to infer a pseudo-temporal trajectory (Fig. 3E). In the Palantir space, nearby points indicate similar persistence images, thereby assuming a gradual transition in their morphologies and the continuous sequence of points define a trajectory. The developmental trajectories were similar between brain regions, with P7 and P22 clusters being the furthest from and the closest to the adult, respectively. In contrast, P15 shifted laterally from the P7-P22 trajectory and occupied the outermost position in nearly all the brain regions, indicating a unique microglial context-dependent response that coincides with neuronal circuit synapse refinement (Kroon et al., 2019; Wong et al., 2005; Yang et al., 2014).

\section{The correlation between microglial phenotypic spectrum and reactivity is region specific in 5xFAD}

Synaptic loss combined with amyloid plaque deposition are common signs of Alzheimer's disease, with the neocortex and the hippocampus being the most affected brain regions (Serrano-Pozo et al., 2011; Terry, 2000). Microglial morphology alters during the progression of Alzheimer's disease (Hemonnot et al., 2019; Taipa et al., 2018) but the disease phenotype of microglia in directly- and indirectly-affected brain regions, as well as the impact on the sexual dimorphism, is not well understood (Congdon, 2018; Gamache et al., 2020; Manji et al., 2019). To address this with MorphOMICs, we traced microglial morphologies in the 5xFAD mouse model, which recapitulates a familial form of Alzheimer's disease (Fig. 4A, 
Supp. Fig. 6A) (Oakley et al., 2006). We traced morphologies for all seven brain regions and focused on animals that were three and six months old $\left(5 \mathrm{xFAD}_{3 \mathrm{~m}}\right.$ and $5 \mathrm{xFAD}_{6 \mathrm{~m}}$, respectively, Supp. Fig. 6B) because amyloid plaques occur first in the deep cortical layers at three months, followed by the hippocampus, coinciding with spine loss and memory deficits around 6 months (Oakley et al., 2006). As anticipated, microglia in the 5xFAD $3 \mathrm{~m}$ group exhibited a disease phenotype in which all brain regions were distinguishable from controls. The $5 \mathrm{xFAD}_{6 \mathrm{~m}}$ group formed a "disease-associated cluster" in the UMAP space, with the exception of $\mathrm{CB}_{\mathrm{mg}}$ (Fig. 4B). $\mathrm{FC}_{\mathrm{mg}}, \mathrm{S}_{\mathrm{mg}}$, and $\mathrm{DG}_{\mathrm{mg}}$ in $5 \mathrm{xFAD}_{3 \mathrm{~m}}$ mice already occupied this disease-associated cluster. By subtracting representative bootstrapped persistence images, we observed that microglia from $5 \mathrm{xFAD}_{6 \mathrm{~m}}$ have more short and long persistent processes close to the soma compared to controls (Supp. Fig. 6C). Moreover, the $5 \mathrm{xFAD}_{6 \mathrm{~m}}$ - control TMD distances reflect the relative phenotypic changes in the UMAP space in Fig. 4B.

When we included sex in our MorphOMICs analysis, the disease cluster was less pronounced (Fig. 4C). Instead, microglia demonstrated higher morphological heterogeneity in $5 \mathrm{xFAD} 3 \mathrm{~m}$ compared to control, with males and females occupying distinct clusters. When we applied Palantir to identify sex-dependent disease trajectories, we observed sexual dimorphism (Fig. 4D, Supp. Fig. 7A), especially in one of the first affected brain region, $\mathrm{S} 1$. $+\mathrm{S} 1_{\mathrm{mg}}$ seem to

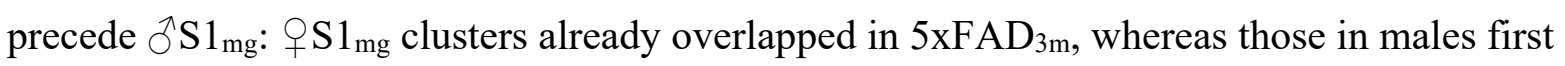
overlapped at 6 months (Fig. 4D). Such a difference in overlap was less obvious for $\mathrm{FC}_{\mathrm{mg}}$ and $\mathrm{DG}_{\mathrm{mg}}$, which is likely influenced by the limited number of selected time points over the course of the pathology. On the other hand, both regions display a phenotypic spectrum along the disease trajectory. To link microglial phenotype to their reactivity, we performed immunostaining for the endosomal-lysosomal marker CD68 (Chistiakov et al., 2017). We then computed the fold-change compared to the control CD68 volume within Iba1 ${ }^{+}$-cells and overlaid the CD68 fold-change on the Palantir trajectory (Fig. 4D). In $q \mathrm{~S} 1_{\mathrm{mg}}, \mathrm{CD} 68$ increased already at 3 months while this only occurred in ${ }^{7} \mathrm{~S} 1_{\mathrm{mg}}$ at 6 months, confirming that the shift along the morphological spectrum happens earlier in females. For the other brain regions, this effect was less obvious. In $\mathrm{FC},{ }^{\lambda} \mathrm{FC}_{\mathrm{mg}}$ appeared to have a reactive microglial state at 3 months based on the CD68 staining, however their morphology overlapped with the control. In contrast, ${ }_{+} \mathrm{FC}_{\mathrm{mg}}, 5 \mathrm{xFAD} 3 \mathrm{~m}$ phenotype segregated from the control, although the CD68 was only upregulated in $5 \mathrm{xFAD}_{6 \mathrm{~m}}$. In the $\mathrm{DG}$, ${ }_{+} \mathrm{DG}_{\mathrm{mg}}$ moved along the disease trajectory faster than ${ }^{\lambda} \mathrm{DG}_{\mathrm{mg}}$, as for $\mathrm{S} 1_{\mathrm{mg}}$, while the $\mathrm{CD} 68$ response in $\mathrm{DG}_{\mathrm{mg}}$ is closer to that of $\mathrm{FC}_{\mathrm{mg}}$. We also applied Palantir trajectories to the other brain regions, since plaques 
deposition has been reported in the olfactory bulb and brainstem (Struble and Clark, 1992). We found a strong sexual dimorphism in microglial morphology in these brain regions, with less-obvious trajectory changes (Supp. Fig. 7C). $\mathrm{CB}_{\mathrm{mg}}$ was the only exception, remaining mainly unaffected in 5xFAD mice, which is consistent with previous literature (Oakley et al., 2006). Overall, the 5xFAD data indicate that the link between microglial disease phenotype and reactivity state depends on the brain region.

\section{Female microglia exhibit an earlier shift along the morphological spectrum in a model of sporadic neurodegeneration}

An alternative model with faster onset and disease progression is the CK-p25 model for sporadic Alzheimer-like degeneration (Cruz and Tsai, 2004; Cruz et al., 2003; Fischer et al., 2005). Upon doxycycline withdrawal, p25 expression is induced in CamKII ${ }^{+}$forebrain neurons, resulting in neurotoxic activity of the cyclin-dependent kinase Cdk6 (Camins et al., 2006). Within two weeks, CK-p25 mice develop progressive neuronal and synaptic loss, forebrain atrophy, aberrant amyloid-precursor protein processing, hyper-phosphorylation of tau (Cruz et al., 2003, 2006), and at later stages neurofibrillary tangle-like pathology (Cruz et al., 2003, 2006) (Fig. 5A). We reconstructed microglial morphologies from CK-p25 mice at 1, 2, and 6 weeks (CK-p25 $5_{1 w}$, CK-p25 2 , CK-p256w, respectively, Supp. Fig. 8A-B) and applied MorphOMICs. Similar to 5xFAD, all seven brain regions started to segregate from the control at 1 week and occupied a disease-associated cluster in CK-p25 $\mathrm{CN}_{\mathrm{mg}}$ staying distinct (Fig. 5B). In particular, $\mathrm{FC}_{\mathrm{mg}}$ reached this cluster already at 2 weeks, while $\mathrm{OB}_{\mathrm{mg}}, \mathrm{DG}_{\mathrm{mg}}, \mathrm{S} 1_{\mathrm{mg}}$, and $\mathrm{SN}_{\mathrm{mg}}$ only at 6 weeks. As in $5 \mathrm{xFAD}$, we confirmed that microglia during CK-p25 have more short and long processes close to the soma compared to controls (Supp. Fig. 8C), and that the CK-p256w - control TMD distances reflect the relative phenotypic changes in the UMAP space in Fig. 5B.

When we applied MorphOMICs to each sex individually, we found that $q$ (SN, FC, OB, DG, and $\mathrm{S} 1)_{\mathrm{mg}}$ reached the disease cluster at 6 weeks, while in males, ${ }^{\lambda} \mathrm{DG}_{\mathrm{mg}}$ and ${ }^{\lambda} \mathrm{S} 1_{\mathrm{mg}}$ stayed distinct (Fig. 5C). Palantir replicated the UMAP and displayed an arm along the trajectory, on which microglial morphology from later disease stages accumulated (Supp. Fig. 9A). We note that neither $\mathrm{CN}_{\mathrm{mg}}$ nor $\mathrm{CB}_{\mathrm{mg}}$ reached this disease-associated arm as expected, due to the low expression of CamKII in these brain regions (Wang et al., 2013). Comparison of the sexspecific Palantir projections also showed that $Q \mathrm{FC}_{\mathrm{mg}}$ preceded ${ }^{\lambda} \mathrm{FC}_{\mathrm{mg}}$ in $\mathrm{CK}-\mathrm{p} 25_{2 \mathrm{w}}$ (Fig. 5D). We could replicate the same dynamics with Monocle, an alternative algorithm which 
uses reverse graph embedding to infer a pseudo-time trajectory (Supp. Fig. 9B) (Trapnell et al., 2014).

When we overlaid the CD68 fold-change compared to control adults over the Palantir $\mathrm{FC}_{\mathrm{mg}}$ trajectory, we found that the CD68 fold-change gradually increased in ${ }^{\lambda} \mathrm{FC}_{\mathrm{mg}}$, while ${ }_{+} \mathrm{FC}_{\mathrm{mg}}$ in CK-p256w showed a sudden increase (Fig. 5D). We also observed that the morphology did not correspond to $\mathrm{CD} 68$ in $q \mathrm{DG} \mathrm{mg}$ and ${ }_{\mathrm{S}} \mathrm{S} 1_{\mathrm{mg}}$ at 6 weeks: the morphology reached the disease-associated arm but did not show any increased CD68 fold change. Neither ${ }^{\Uparrow} \mathrm{DG}_{\mathrm{mg}}$ nor $\stackrel{\AA}{S} 1_{\mathrm{mg}}$ reached the disease-associated arm, which we also confirmed with Monocle (Supp. Fig. 9B). On the other hand, ${ }^{\lambda} \mathrm{DG}_{\mathrm{mg}}$ and ${ }^{\mathrm{D}} \mathrm{DG}_{\mathrm{mg}}$ showed their highest $\mathrm{CD} 68$ fold change at 2 weeks and occupied a similar cluster in the Palantir space (Fig. 5D). This suggests that the microglial response might be associated with the transient effect of p25 expression, which has been shown to enhance long-term potentiation and improve hippocampus-dependent memory, before inducing neurodegeneration, gliosis, and severe cognitive decline at 6 weeks (Fischer et al., 2005). For those brain regions that were less affected, dimorphic microglial phenotype was less pronounced (Supp. Fig. 9C). In both sexes, $\mathrm{SN}_{\mathrm{mg}}$ and $\mathrm{OB}_{\mathrm{mg}}$ in $\mathrm{CK}-\mathrm{p} 25_{6 \mathrm{w}}$ reached the disease-associated arm, whereas in $\mathrm{CB}_{\mathrm{mg}}$ and $\mathrm{CN}_{\mathrm{mg}}$, neither sex nor disease progression influenced morphology (Supp. Fig. 9D). Overall, the CK-p25 model exhibited strong dimorphic phenotype spectrum in favor of females, which precede their male counterparts in a brain-region-specific manner.

\section{Microglial phenotype during early developmental integrates into the disease spectrum}

Until now, we have treated microglial morphology separately for development and disease. Since both the development and the disease phenotypes result from a shift along the morphological spectrum, we were interested in how these conditions integrate along the pseudo-temporal trajectory. To achieve this, we performed MorphOMICs for all brain regions, including developmental time point $\mathrm{P} 7,5 \mathrm{xFAD}$, and $\mathrm{CK}-\mathrm{p} 25$ for each sex separately and extracted the trajectory with Palantir. We first focused on the female trajectory for CKp25 $5_{\mathrm{w}}$ in $q \mathrm{FC}_{\mathrm{mg}}$ and ${ } \mathrm{DG}_{\mathrm{mg}}$ identifying the control-to-disease spectrum (Fig. 6A). In ${ }_{+} \mathrm{FC}_{\mathrm{mg}}$, the $\mathrm{P} 7,5 \mathrm{xFAD} 3 \mathrm{~m}$, and $5 \mathrm{xFAD}_{6 \mathrm{~m}}$ groups formed a continuum with the latter reaching out towards the CK-p25 $2 \mathrm{w}$ group. Interestingly, ${ } \mathrm{DG}_{\mathrm{mg}}$ mimicked a similar trajectory but with both 5xFAD and early CK-p25 forming a cluster distant from the control, and P7 group reaching out towards $\mathrm{CK}-\mathrm{p} 25_{6 \mathrm{w}}$. All ${ }_{+} \mathrm{CB}_{\mathrm{mg}}$, which is the less affected region during neurodegeneration, segregate in the Palantir space, excluding P7. 
In males, disease phenotypes evolved more slowly than in females, with only $\overbrace{}^{\lambda} \mathrm{FC}_{\mathrm{mg}}$ reaching the disease-associated arm at CK-p25 both 5xFAD groups close to the control, followed by intermingled P7, CK-p251w and,

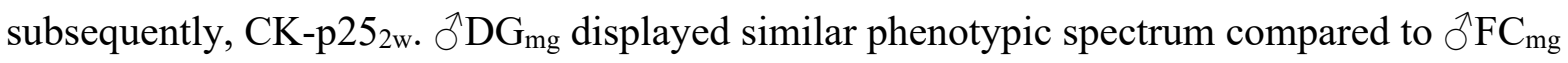
for both 5xFAD groups, shifting towards the CK-p252w-CK-p256w cluster. $\overbrace{}^{\lambda} \mathrm{CB}_{\mathrm{mg}}$ behaved similarly to females in the 5xFAD model (Fig. 4C). When we looked at the indirectly affected brain regions, the control-to-disease spectrum was less prominent (Supp. Fig. 10AB). Overall, our data show that microglia display a spectrum of phenotypes, with P7 occupying distinct parts of the trajectory in a brain-region-dependent manner. This suggests that the microglial phenotype is comparable between normal development and disease, which are both environments with neuronal circuit remodeling.

\section{MorphOMICs maximizes the extraction of morphological information}

MorphOMICs enabled us to establish both an adult sexual dimorphic phenotype and a morphological spectrum during development and degeneration for each brain region. Such a reconstruction is not straightforward with common morphological feature selection (Supp.

Fig. 1A). We could not replicate the sexually dimorphic control-to-disease spectrum from Fig. 5D on microglia from females and males in CK-p25 mice with statistical comparisons of process length, number of branches, and terminal, and branching points (Supp. Fig. 11A). We also lost information of the morphological spectrum when we applied bootstrapping approaches to the extended set of non-interdependent morphometric quantities on the CK-p25 $\mathrm{FC}_{\mathrm{mg}}$ (Fig. 7A). The same loss was observed applying the bootstrap method to microglia in the $5 x F A D$ model and during development (Supp. Fig. 11B-C). This suggests that MorphOMICs preserves certain intrinsic properties of the reconstructed tree after dimensionality reduction.

To identify which properties are potentially relevant, we looked at the most variable pixels across CK-p25 $\mathrm{FC}_{\mathrm{mg}}$ and the control bootstrapped persistence images (Fig. 7C). We found the highest variability along the diagonal and close to origin of the persistence diagram corresponding to short branches and branches close to the soma (see also Fig. 1B, v). We therefore decided to zoom in on the short and the long persistence bars, filtered them out separately, and repeated our MorphOMICs analysis (Fig. 7D). Using this method, we saw that just a few long bars sufficed to capture the sexually dimorphic phenotypes along the disease trajectory (Fig. 7E, F). In contrast, we observed a different dimorphic control-to- 
disease spectrum if we focused only on the short bars (Fig. 7G). These results suggest that persistence barcodes highlight different phenomena, and therefore both short and long bars are essential for the understanding of morphology.

\section{$\underline{\text { Discussion }}$}

In this study, we analyzed heterogeneity and sexual dimorphism of microglia morphology across seven brain regions from a total of 39,190 cells through development and disease (Supp. Table 1). To do so, we developed and applied the MorphOMICs pipeline, which extracts the information of the entire reconstructed microglial tree in a minimally biased way, combined with variability reduction and improved data visualization.

\section{Insights into the microglial phenotypic spectrum}

Microglial functional heterogeneity has been indicated using brain-region-specific single-cell transcriptome analysis (Furube et al., 2018; Grabert and McColl, 2018; Mildner et al., 2011), but morphological differences have been difficult to identify and quantify. MorphOMICs revealed that the microglia in an adult brain exhibit regional heterogeneity (Fig. 1F) that exists already in early postnatal development (Fig. 3C) and diminishes during degeneration (Fig. 4B, 5B). Although microglia display a phenotypic spectrum (Fig. 6), they respond to disease in a brain-region-specific manner, dependent on the degeneration model. In fact, frontal brain regions are known to be first responders in Alzheimer-like degeneration (Bakkour et al., 2013; Desikan et al., 2010).

Even though we did not directly compare the functional consequences of environmental changes on microglia, we observed some important links. Microglia shifted from the P7/P22 trajectory across all brain regions at P15 (Fig. 3E), which is the time of circuit refinement, where microglia have frequently been shown to participate in the process of synaptic pruning (Hattori and McGeer, 1973; Kroon et al., 2019; Maklad and Fritzsch, 2003; Wong et al., 2005). Another synapse-associated pattern occurred in the $\mathrm{DG}_{\mathrm{mg}}$ of CK-p25 $2 \mathrm{w}$ (Fig. 5D), where we unexpectedly found the highest CD68 fold change and not within the CK-p256w, where we have observed the most distinct morphological shift from the control. This discrepancy might be associated with transient p25 expression, which has been previously described (Fischer et al., 2005). In general, we could associate CD68 upregulation with an increasingly diverse phenotype, indicating an increased reactivity, as exhibited in ${ }^{\circ} \mathrm{FC}_{\mathrm{mg}} \mathrm{CK}-$ p25. However, this effect was less prominent in $\overbrace{}^{\lambda} \mathrm{FC}_{\mathrm{mg}}$ (Fig. 5D), suggesting that CD68 
upregulation is sex-, brain-region-, and context-dependent. This aspect has to be taken into account for future use of CD68 as a reactivity marker.

\section{Sex as confounding factor of microglial phenotypic spectrum}

To what extent sex affects microglial morphology has long been debated (Lenz et al., 2013; Nelson et al., 2017). Here, we confirmed that a sex-specific phenotype exists, which as we have shown is rather mild during adulthood (Fig. 2B) but prominent during development (Fig. 3D) and degeneration (Fig. 4C, 5C). We found a sexually dimorphic microglial response in both degeneration models, which was pronounced in the immediately affected brain regions $\mathrm{FC}_{\mathrm{mg}}, \mathrm{DG}_{\mathrm{mg}}$, and $\mathrm{S} 1_{\mathrm{mg}}$. Interestingly, females showed an earlier shift along the morphological spectrum compared to males. This supports studies that have suggested a sexdependent difference in Alzheimer's disease progression (Congdon, 2018; Gamache et al., 2020; Manji et al., 2019) and points to females having a higher risk of developing dementia (Payami et al., 1996; Turner, 2001).

Estrogens have been shown to be involved in the masculinization of the brain (Lenz and McCarthy, 2015; Lenz et al., 2013; Nissen, 2017), and microglia are also suspected of playing a role in this process (Lenz and McCarthy, 2015; Lenz et al., 2013). We were surprised to see that microglia from the ovariectomized females were distinct from their nonovariectomized counterparts, but also that brain regions intermingled differently (Fig. 2B-C). Whereas $\rho_{F} C_{\mathrm{mg}}$ and $\rho_{\mathrm{OB}} \mathrm{B}_{\mathrm{mg}}$ occupied a similar cluster in control adults, $\rho_{\text {ov }} \mathrm{FC}_{\mathrm{mg}}$ were distinct from $q_{\text {ov }} \mathrm{OB}_{\mathrm{mg}}$ and highly intermingled with $q_{\mathrm{ov}} \mathrm{SN}_{\mathrm{mg}}$ (Fig. 2C), suggesting that the impact of estrogens on microglial morphology is complex. Overall, MorphOMICs links the previously reported sexually dimorphic microglial transcriptome in the healthy brain (Ayata et al., 2018; Schwarz et al., 2012; Thion et al., 2018; Villa et al., 2018) and in degeneration models (Bruce-Keller et al., 2000; Crain and Watters, 2010; Vegeto et al., 2006; Villa et al., 2015, 2016; Yanguas-Casás et al., 2018) with a distinct morphological phenotype.

\section{MorphOMICs as a novel tool for morphological analysis}

An advantage of MorphOMICs is that it preserves the intrinsic properties of the reconstructed morphological tree and avoids feature-selection-derived biases. Interestingly, we found that both short and long bars within a barcode contain information that contribute to microglial spectrum (Fig. 7E-G). Future studies will focus on identifying particularly informative regions of a persistence barcode, which provides a perspective for morphological analysis of 
lower-resolution images, such as in vivo microglial imaging for potential non-invasive diagnostic applications. Stable ranks would provide a mathematically robust approach to address this question, as we have shown already that standard stable ranks of the TMD captured the microglial phenotypes as well as the persistence images of the microglial TMD (Supp. Fig. 2G) (Riihimäki and Chacholski, 2018).

MorphOMICs provides an advanced strategy for systematically comparing microglial populations across different brain regions and conditions: this could be expanded infinitely. We therefore provide our dataset in NeuroMorpho.org and the code as a download on GitHub (https://git.ist.ac.at/rcubero/morphomics), with detailed instructions on implementation. A critical point to consider is the number of cells that are needed for MorphOMICs. While we identified a suitable bootstrap size in Supp. Fig. 2D, the condition-specific variability in microglial morphology needs to be systematically assessed to determine the minimum cell number before MorphOMICs can be reliably applied.

Most important, MorphOMICs overcomes the dichotomized view of microglial morphology to either ramified, relating to a surveilling function, or amoeboid, for highly phagocytosing. This is relevant because microglia present a wide spectrum of morphological responses to local cues that are neglected with classical analysis, thus compromising the use of morphology as a readout of environmental modification.

\section{Figure legends}

Figure 1. MorphOMICs dissects microglial morphology in adult healthy brains.

A: Sagittal view of the murine brain (Image credit: Allen Institute) with annotated brain regions: olfactory bulb $(\mathrm{OB})$, frontal cortex $(\mathrm{FC})$, dentate gyrus $(\mathrm{DG})$, somatosensory cortex $(\mathrm{S} 1)$, substantia nigra $(\mathrm{SN})$, cochlear nucleus $(\mathrm{CN})$, and cerebellum $(\mathrm{CB})$. Next, confocal images of immunostained microglia (Iba1, allograft inflammatory factor 1, green) and cell nuclei (Hoechst, blue) from adult C57BL/6J mice with zoom-in. Scale bar: $50 \mu \mathrm{m}$. B: Schematic of MorphOMICs pipeline covering topological morphology descriptor (TMD). Each traced microglia is converted into a rooted tree (i, start (\#) and end $(*)$ of longest process in red), and from there into a persistence barcode (ii), a persistence diagram (iii, with each bar collapsed to a point), and a persistence image (iv) with grey-scaled process density in space. Blue spot: soma location. Arrow 1 indicates distance from the soma and arrow 2 process length, which increases the further away from the diagonal. $\mathrm{n}$ persistence images are 
summarized to an average persistence image. C: Average persistence images of the seven analyzed brain regions organized by hierarchical clustering (see also Supp. Fig. 1B). Top corner: representative microglia. The darker the green, the higher the frequency distribution of the processes. D: Schematic of MorphOMICs pipeline covering bootstrapping. Microglial population (n) contains individual persistence images. In the center: average persistence image. $\mathrm{x}$ unique persistence images are drawn from each of $\mathrm{n}$ microglial pools to generate a bootstrapped persistence image. Repeating this process $\mathrm{m}$ times forms the bootstrapped pool. E: Schematic of MorphOMICs pipeline covering dimension reduction and data visualization with Uniform Manifold Approximation and Projection (UMAP). Left: each persistence image is pixelated, and each pixel represents a dimension. Middle: reducing dimensions with principal component (PC) analysis. Right: further dimensionality reduction based on the first ten PCs. F: UMAP plot of MorphOMICs-analyzed microglia, color-coded for each brain region. Each dot represents a bootstrapped persistence image (D). Data for each brain region from at least six animals, both sexes together.

Figure 2. MorphOMICs identifies sexually-dimorphic microglial morphology in healthy adults.

A: Sagittal view of analyzed brain regions: olfactory bulb $(\mathrm{OB})$, frontal cortex $(\mathrm{FC})$, dentate gyrus (DG), somatosensory cortex (S1), substantia nigra (SN), cochlear nucleus $(\mathrm{CN})$, and cerebellum (CB) (Image credit: Allen Institute (Lein et al., 2006)). B: UMAP plot of MorphOMICs-analyzed microglia for each brain region color-coded for males (left) or females (right) with dashed lines vice versa as reference. Each dot represents a bootstrapped persistence image. $\mathrm{CN}_{\mathrm{mg}}: \mathrm{t}=3.504, \mathrm{df}=15.1, \mathrm{p}$-value $=0.00312 . \mathrm{OB}_{\mathrm{mg}}: \mathrm{t}=2.401, \mathrm{df}=$ 16.864, $\mathrm{p}$-value $=0.0282 . \mathrm{CB}$ g: $\mathrm{t}=1.2564, \mathrm{df}=17.327, \mathrm{p}$-value $=0.2257 . \mathrm{FC}_{\mathrm{mg}}: \mathrm{t}=$ 1.6236, $\mathrm{df}=16.275, \mathrm{p}$-value $=0.1237 . \mathrm{SN}_{\mathrm{mg}}: \mathrm{t}=1.6261, \mathrm{df}=12.901, \mathrm{p}$-value $=0.1281 . \mathrm{DG}$ $\mathrm{mg}: \mathrm{t}=0.68892, \mathrm{df}=19.669$, $\mathrm{p}$-value $=0.4989 . \mathrm{S} 1_{\mathrm{mg}} \mathrm{t}=1.5618, \mathrm{df}=17.518, \mathrm{p}$-value $=$ 0.1362. $\mathrm{n}_{\varphi}=11, \mathrm{n}_{\delta}=11$. C: UMAP plot of MorphOMICs-analyzed microglia in ovarectomized females. Ovariectomized brain regions are highlighted and the nonovarectomized counterpart is shown in grey as a reference (see also Supp. Fig. 3B).

Figure 3. Microglial phenotypes during postnatal development. 
A: Left: schematic of postnatal brain development with key aspects of brain maturation and neuronal circuitry refinement. Right: sagittal view of analyzed brain regions: olfactory bulb (OB), frontal cortex (FC), dentate gyrus (DG), somatosensory cortex (S1), substantia nigra $(\mathrm{SN})$, cochlear nucleus $(\mathrm{CN})$ and cerebellum $(\mathrm{CB})$. (Image credit: Allen Institute (Lein et al., 2006)). B-D: UMAP plots of MorphOMICs-analyzed microglia across seven brain regions in Cx3cr1-GFP ${ }^{+/-}$mice (B) at P7 (light brown), P15 (orange), P22 (dark brown), and adults (dark grey); and color-coded brain regions for both sexes $(\mathbf{C})$ and for each sex independently with dashed lines vice versa as reference (D) for each developmental time point. Each dot represents a bootstrapped persistence image. E: Palantir reconstruction of microglia morphological trajectory from (D) with highlighted P7 (light brown), P15 (orange), P22 (dark brown) and adults (dark grey) for each brain region.

\section{Figure 4. Microglia phenotypic spectrum in 5xFAD transgenic model of neurodegeneration is sexually dimorphic.}

A: Left: sagittal view of analyzed brain regions: olfactory bulb (OB), frontal cortex (FC), dentate gyrus (DG), somatosensory cortex (S1), substantia nigra (SN), cochlear nucleus $(\mathrm{CN})$ and cerebellum (CB). (Image credit: Allen Institute (Lein et al., 2006)). Right: timeline of degeneration events in 5xFAD transgenic mouse model. B-C: UMAP plots of MorphOMICsanalyzed microglia across seven brain regions for the following conditions: control, $5 \mathrm{xFAD} 3 \mathrm{~m}$ (3 months), and 5xFAD 6 m (6 months) with both sexes (B) or for each sex separated (C). Each degeneration time point is highlighted in a separate UMAP. Each dot represents a bootstrapped persistence image. D: Representative confocal images of microglia (Iba1, green) and lysosome (CD68, magenta), followed by Palantir reconstruction of microglial trajectory with corresponding color-coded CD68 fold change below from females (left side) and males (right side) for control, $5 \mathrm{xFAD}_{3 \mathrm{~m}}$, and $5 \mathrm{xFAD}_{6 \mathrm{~m}}$ in $\mathrm{S} 1, \mathrm{FC}$, and DG. Scale bar: 10 $\mu \mathrm{m}$. Fold change $<0$ blue; $>0$ red.

\section{Figure 5. The microglia phenotype of females in CK-p25 model of neurodegeneration exhibit an earlier morphological shift than in males}

A: Left: sagittal view of analyzed brain regions: olfactory bulb (OB), frontal cortex (FC), dentate gyrus (DG), somatosensory cortex (S1), substantia nigra (SN), cochlear nucleus (CN) and cerebellum (CB). (Image credit: Allen Institute (Lein et al., 2006)). Right: timeline reporting neurodegeneration events upon doxycycline withdrawal in the CK-p25 transgenic 
mouse model. B-C: UMAP plots displaying microglial morphological heterogeneity in adult control mice and CK-p25 mice 1, 2 and 6 weeks after doxycycline withdrawal across all the analyzed brain regions for both sexes $(\mathbf{B})$ or for each sex separated $(\mathbf{C})$. Each dot represents a bootstrapped persistence image, and each UMAP highlights a distinct degeneration time point. D: Representative confocal images of microglia (Iba1, green) and lysosome (CD68, magenta) from females (left) and males (right) in CK-p25 mice 1, 2, and 6 weeks after doxycycline withdrawal in S1, FC, and DG. Scale bar: $10 \mu \mathrm{m}$. Palantir reconstruction of microglial morphological trajectory and corresponding color-coded CD68 fold change of female (central column, left) and male (central column, right). Fold change $<0$ blue; $>0$ red.

\section{Figure 6. Early developmental phenotype integrates into the disease spectrum}

A-B: Palantir reconstructions of microglial trajectory in females (A) and males (B) for FC, DG, and CB in control (grey), P7 (light brown), CK-p251w (1 week, light green), CK-p25 ww (2 weeks, green), CK-p25 6 (6 weeks, dark green), 5xFAD $3 \mathrm{~m}$ (3 months, light purple), and $5 \mathrm{XFAD}_{6 \mathrm{~m}}(6$ months, purple) conditions. Each brain region is highlighted in a separate Palantir plot. Black arrow: control-to-disease spectrum.

\section{Figure 7. MorphOMICs applied to primary processes reiterates sexual dimorphism in CK-p25 mice}

A: Left: box plots for the selected features dendritic length, number of branches, and terminal and branching points of control $\left(\mathrm{n}_{+}=926, \mathrm{n}_{\delta}=894\right)$, and CK-p25 $5_{1 \mathrm{w}}\left(\mathrm{n}_{+}=219, \mathrm{n}_{\delta}=194\right), \mathrm{CK}-$ $\mathrm{p} 25_{2 \mathrm{w}}\left(\mathrm{n}_{+}=264, \mathrm{n}_{\delta}=492\right)$, CK-p256w $\left(\mathrm{n}_{+}=858, \mathrm{n}_{\delta}=462\right)$ mice $(1-, 2-$ and 6-weeks after doxycycline withdrawal, respectively) in the frontal cortex (FC). Right: matrices showing color-coded p-values for the pairwise comparison of each morphometric A: UMAP representations based on multiple classical morphometrics (see Supp. Table 4) during CKp256w degeneration in females (left) and males (right). Highlighted clusters are FC for control (dark grey), CK-p25 1w (1-week, light green), CK-p25 2w (2 weeks, green), CK-p25 6 w (6 weeks, dark green). B: Heat map showing the pixel-wise standard variation of the bootstrapped persistence images across control and CK-p25 conditions. C: Schematic for filtering persistence barcodes with MorphOMICs. Starting from microglial rooted tree, only bars are selected that are born at $0 \mu \mathrm{m}$ and independent of their length (representing likely primary branches). These bars are converted into a persistence diagram. D-F: Palantir 
trajectory of all brain region (without cochlear nucleus and cerebellum for simplicity) from control and CK-p25 condition with highlighted FC microglia trajectory for females (left) and males (right) with (D) unfiltered or filtered bars (E: start radial distance from the soma $=$ $0 \mu \mathrm{m}, \mathbf{F}$ : maximum bar length $=10 \mu \mathrm{m}) .{ }^{*} \mathrm{p}<0.05, * * \mathrm{p}<0.01$.

\section{$\underline{\text { Supplementary Figures }}$}

\section{Supplementary Figure 1. Classic morphometry analysis and intrinsic variability of microglial morphology.}

A: Box plots for the following morphometric features: process length, number of branches, and terminal and branching points for traced adult $\mathrm{C} 57 \mathrm{BL} / 6 \mathrm{~J}$ microglia across different brain regions: CB (cerebellum, $n=299$ ), $\mathrm{CN}$ (cochlear nucleus, $\mathrm{n}=498$ ), FC (frontal cortex, $\mathrm{n}=926$ ), DG (dentate gyrus, $\mathrm{n}=902$ ), OB (olfactory bulb, $\mathrm{n}=796$ ), $\mathrm{S} 1$ (somatosensory cortex, $\mathrm{n}=719$ ), $\mathrm{SN}$ (substantia nigra, $\mathrm{n}=1050$ ) from at least six animals. Next, matrices with color-coded $\mathrm{p}$ values for the pairwise comparison of each morphometric (see also Supp. Table 2). B: Hierarchically-ordered heat map for pairwise TMD intrinsic distances between average persistence images from microglia across brain regions from Fig. 1A. C-D: UMAP plots of the entire microglial population size (grey) with color-highlighted brain regions $(\mathbf{C})$ or animals (D). Each dot represents a single persistence image. (D) Triangle and circle for females and males, respectively. ${ }^{*} \mathrm{p}<0.05,{ }^{* *} \mathrm{p}<0.01$.

\section{Supplementary Figure 2. Details about the MorphOMICs paradigm.}

A: Schematic of the bootstrapping effects on the distance between tree structures from the same population (within-population distance, green arrows) and two distinct populations (distance between average persistence images, purple lines). Increase of bootstrap-topopulation size ratio $(\mathrm{x} / \mathrm{n})$ reduces within-population distance and increases distance between average persistence images. B: UMAP plots of MorphOMICs-analyzed microglia for frontal cortex (orange) and dentate gyrus (yellow) for different bootstrap-to-population size ratios. Left: $\mathrm{x}=1$, allows no segregation. Middle: $\mathrm{x} / \mathrm{n}=0.3$. Right: $\mathrm{x} / \mathrm{n}=1$ causes accentuation. $\mathrm{C}$ : Line plot \pm SD displays how within-population distance (top) and mixing entropy (bottom) decrease with enhanced bootstrap-to-population size ratio (x/n). An empirical threshold of 0.3 was selected (red dashed line). D: Line plot \pm SD displays how the ratio between mixing 
entropy and within-condition distance varies by enhancing the bootstrap-to-population size ratio (x/n). E: UMAP plots of MorphOMICs-analyzed microglia for frontal cortex (green) and dentate gyrus (purple) for different bootstrap-to-population size ratios and varying maleto-female ratios within the population size. Olfactory bulb $(\mathrm{OB})$, frontal cortex $(\mathrm{FC})$, dentate gyrus (DG), somatosensory cortex (S1), substantia nigra (SN), cochlear nucleus (CN), and cerebellum (CB). F: UMAP plots of MorphOMICs-analyzed microglial morphology across seven brain regions as shown for Fig. 1F with varying hyperparameters for number of neighbors (n_neighbors), minimum distance (min_dist), and spread. G: t-SNE plot of MorphOMICs-analyzed microglia across seven brain regions as shown in Fig. 1F for UMAP. H: UMAP plots of stable-rank representation of microglial morphology (see Methods: Stable Ranks) across seven brain regions. SD: standard deviation.

\section{Supplementary Figure 3. Sholl analysis of microglial heterogeneity depends on the Sholl step size radius.}

A: Heatmap of classification accuracy between pairs of brain regions using stable ranks for different bootstrap sizes. Numbers indicates the percentage of microglia correctly assigned in the classification task, averaged over 10 repeated cross-validations. 1, perfect assignment; 0.5 random assignment. B: Sholl curves showing the number of processes, averaged per each control brain region $\pm \mathrm{SD}$, that intersect with a series of concentric Sholl spheres spaced at $5 \mu \mathrm{m}$. C: UMAP plot of Sholl-analyzed microglia, color-coded for each brain region. Each dot represents a bootstrapped Sholl analysis, setting the consecutive radius step size at 1, 3, 5, 7, $10 \mu \mathrm{m}$ respectively. SD: standard deviation.

\section{Supplementary Figure 4. Adult microglial density and the impact of ovariectomy on their phenotype.}

A: Bar plot describing the distribution of microglial density across brain regions in C57BL/6J female and male adults. Cell density is expressed as the number of cells per $\mathrm{mm}^{2} \pm \mathrm{SD}$. Sex averages for microglia from each region were compared with two-sided t-test. $\mathrm{CN}_{\mathrm{mg}}(\mathrm{q}=11$, $\hat{\delta}=11 ; \mathrm{t}=3.504, \mathrm{df}=15.1, \mathrm{p}$-value $=0.00317)$ and $\mathrm{OB}_{\mathrm{mg}}(\mathrm{q}=11, \hat{\delta}=11 ; \mathrm{t}=2.401, \mathrm{df}=$ 16.864, $\mathrm{p}$-value $=0.0282)$. B: Confocal images of immunostained microglia (Iba1, green) and cell nuclei (Hoechst, blue) from ovariectomized C57BL/6J adult mice for each brain region with zoom-in. Scale bar: $50 \mu \mathrm{m}$. 
A: Sagittal view of analyzed brain regions: olfactory bulb (OB), frontal cortex (FC), dentate gyrus (DG), somatosensory cortex (S1), substantia nigra (SN), cochlear nucleus $(\mathrm{CN})$ and cerebellum (CB). (Image credit: Allen Institute (Lein et al., 2006)) B: Confocal images of $\mathrm{GFP}^{+}$(green) microglia and cell nuclei (Hoechst, blue) from $\mathrm{Cx} 3 \mathrm{crl}^{+/ \mathrm{GFP}}$ mice at P7, P15, and P22 for each brain region with zoom-in. Scale bar: $50 \mu \mathrm{m}$. C: UMAP plots of MorphOMICsanalyzed microglia from B for females (left) and males (right) at P7 (light brown), P15 (orange), P22 (dark brown), and adults (dark grey). Separate UMAP for each brain region and sex. Each dot represents a bootstrapped persistence image.

\section{Supplementary Figure 6. Microglia phenotypic spectrum in the 5xFAD model of familiar Alzheimer's neurodegeneration.}

A: Sagittal view of analyzed brain regions: olfactory bulb (OB), frontal cortex (FC), dentate gyrus (DG), somatosensory cortex (S1), substantia nigra (SN), cochlear nucleus $(\mathrm{CN})$ and cerebellum (CB). (Image credit: Allen Institute (Lein et al., 2006)) B: Confocal images showing stained microglia (Iba1, green) and cell nuclei (Hoechst, blue) from the analyzed brain regions in $5 \mathrm{xFAD}_{3 \mathrm{~m}}$ and $5 \mathrm{xFAD}_{6 \mathrm{~m}}$ ( 3 and 6 months, respectively) with zoom-in. Scale bar: $50 \mu \mathrm{m}$. C: Representative persistence images corresponding to each cluster centroid from Fig. 4B with color-coded process density (orange $=$ control; blue $=5 \times \mathrm{FAD}_{6 \mathrm{~m}}$ ) and respective subtraction images per every brain region (orange = increased control process density; blue = increased $5 \mathrm{xFAD}_{6 \mathrm{~m}}$ process density)

\section{Supplementary Figure 7. Sexually dimorphic microglia phenotype in 5xFAD}

A: Palantir reconstruction of microglial morphological trajectory in males (top) and females (bottom) from Supp. Fig. 6B. Each time-point highlighted in a separate Palantir plot. B: Palantir reconstruction of microglial trajectory with corresponding color-coded CD68 fold change next to it for females (left) and males (right) in control adult, $5 \times F_{3} D_{3 m}$, and $5 \times \mathrm{xAD}_{6 \mathrm{~m}}$ of $\mathrm{OB}, \mathrm{SN}, \mathrm{CN}$, and $\mathrm{CB}$. Fold change $<0$ blue; $>0$ red.

\section{Supplementary Figure 8 Microglia phenotypic spectrum in the CK-p25 model of sporadic neurodegeneration.}

A: Sagittal view of analyzed brain regions: olfactory bulb (OB), frontal cortex (FC), dentate gyrus (DG), somatosensory cortex (S1), substantia nigra (SN), cochlear nucleus (CN) and cerebellum (CB). (Image credit: Allen Institute (Lein et al., 2006)) B: Confocal images 
showing stained microglia (Iba1, green) and cell nuclei (Hoechst, blue) from analyzed brain regions and CK-p25 $5_{1 w}$, CK-p25 2 , CK-p25 $5_{6 w}$ mice (1-, 2- and 6-weeks after doxycycline withdrawal) with zoom-in. Scale bar: $50 \mu \mathrm{m}$. C: Representative persistence images corresponding to each cluster centroid from Fig. 5B with color-coded process density (orange $=$ control; blue $=\mathrm{CK}-\mathrm{p} 25_{6 \mathrm{w}}$ ) and respective subtraction images per every brain region (orange $=$ increased control process density; blue $=$ increased CK-p256w process density)

\section{Supplementary Figure 9. Sexually dimorphic microglial phenotype in CK-p25}

A: Monocle reconstruction of microglia trajectory of females (left) and males (right) in control, adult and CK-p25 mice FC, DG, and S1. B: Palantir reconstruction of microglial morphological trajectory in males (top) and females (bottom) in control and CK-p25 mice. Each disease time-point highlighted in a separate Palantir plot. C-D: Palantir reconstruction of microglia trajectory and corresponding color-coded CD68 fold-change of females (left) and males (right) from Supp. Fig. 8B for SN, OB (C) and CN, CB (D). Fold change $<0$ blue; $>0$ red.

\section{Supplementary figure 10. Integration of early developmental phenotype into the disease spectrum in secondarily-affected regions}

A-B: Palantir reconstructions of microglia trajectory in females (A) and males (B) for S1, $\mathrm{SN}, \mathrm{OB}$, and CN in control (dark grey), P7 (light brown), CK-p25 $1 \mathrm{w}$ (1-week, light green), CK-p25 2w (2 weeks, green), CK-p256w (6 weeks, dark green), 5xFAD $3 \mathrm{~m}$ (3 months, light purple), and $5 \mathrm{xFAD}_{6 \mathrm{~m}}(6$ months, purple) conditions. Each brain region is highlighted in a separate Palantir plot. Black arrow: control-to-disease spectrum

\section{Supplementary figure 11. Classical morphometric do not recapitulate MorphOMICs observations}

A: Left: box plots for the selected features dendritic length, number of branches, and terminal and branching points of control $\left(\mathrm{n}_{+}=926, \mathrm{n}_{\delta}=894\right)$, and CK-p25 ${ }_{1 \mathrm{w}}\left(\mathrm{n}_{+}=219, \mathrm{n}_{\delta}=194\right), \mathrm{CK}-$ $\mathrm{p} 25_{2 \mathrm{w}}\left(\mathrm{n}_{+}=264, \mathrm{n}_{\delta}=492\right)$, CK-p256w $\left(\mathrm{n}_{+}=858, \mathrm{n}_{\delta}=462\right)$ mice $(1-, 2$ - and 6-weeks after doxycycline withdrawal, respectively) in the frontal cortex (FC). Right: matrices showing color-coded p-values for the pairwise comparison of each morphometric. B-C: UMAP 
representations based on an extended list of morphometrics (see Supplementary Table 4.) applied to females (left) and males (right) in 5xFAD (B) and Cx3cr1-GFP ${ }^{+/-}$mice (C). Highlighted clusters are FC for control/adult (dark grey), 5xFAD $3 \mathrm{~m}$ (3 months, light purple), $5 \mathrm{xFAD} 3 \mathrm{~m}$ (6 months, dark purple), P7 (light brown), P15 (orange) and P22 (dark brown) respectively.

Supplementary Table 1. Number of traced microglia for each brain region, sex, and condition

\section{Supplementary Table 2. Statistical tests related to Supplementary Figure 1A.}

P-value for pairwise comparisons between adult microglia of a brain regions for each morphometric. Cerebellum (CB), cochlear nucleus $(\mathrm{CN})$, dentate gyrus (DG), frontal cortex (FC), olfactory bulb (OB), somatosensory cortex (S1), substantia nigra (SN).

\section{Supplementary Table 3. Statistical tests related to Figure 7A.}

P-value for pairwise comparison between microglia from the frontal cortex of the CK-p25 for different conditions and sex.

\section{Supplementary Table 4. Classical morphometric related to Figure 7A, Supplementary Figure 11B-C.}

Extended list of classical morphometric quantities (Bijari et al., 2021; Polavaram et al., 2014).

\section{Material and Methods}

Animals. C57BL/6J (Cat\#000664) and B6.129P-Cx3cr1tm1Litt/J (Cat\#005582, named here $\mathrm{Cx} 3 \mathrm{cr}^{\mathrm{GFP} /}$, only heterozygous were used) were purchased from The Jackson Laboratories. All animals were housed in the IST Austria Preclinical Facility, with 12 hours light-dark cycle, food and water provided ad libitum. Animal from both sexes were used, as indicated in the results. All animal procedures are approved by the Bundesministerium für Wissenschaft, Forschung und Wirtschaft (bmwfw) Tierversuchsgesetz 2012, BGBI. I Nr. 114/2012, idF BGBI. I Nr. 31/2018 under the numbers 66.018/0005-WF/V/3b/2016, 66.018/0010$\mathrm{WF} / \mathrm{V} / 3 \mathrm{~b} / 2017,66.018 / 0025-\mathrm{WF} / \mathrm{V} / 3 \mathrm{~b} / 2017,66.018 / 0001 \_\mathrm{V} / 3 \mathrm{~b} / 2019,2020-0.272 .234$. 
5xFAD and CK-p25 mice were obtained from the Tsai lab at MIT. All animal work was approved by the Committee for Animal Care of the Division of Comparative Medicine at the Massachusetts Institute of Technology. 5xFAD mice (B6SJLTg(APPSwFILon,PSEN1*M146L L286V)6799Vas/Mmjax, Stock No: 34840-JAX) were obtained from Jackson laboratory. CK-p25 mice(Cruz et al., 2003),(Cruz et al., 2006)'(Fischer et al., 2005) were generated by breeding CaMKII $\alpha$ promoter-tTA mice (CK controls) (B6;CBA-Tg(Camk2a-tTA)1Mmay/J, Jackson Laboratory, Stock No: 003010) with tetOCDK5R1/GFP mice (C57BL/6-Tg(tetO-CDK5R1/GFP)337Lht/J, Jackson Laboratory, Stock No: 005706). CK-p25 mice and their CK control littermates were conceived and raised in the presence of doxycycline-containing food to repress p25 transgene expression. To induce p25 transgene expression, mice were fed a normal rodent diet. p25 transgene expression was induced in adult mice at the age of 3 months. Since it is known that doxycycline acts as inhibitor of microglial activation(Jantzie et al., 2005; Santa-Cecília et al., 2016) and keep microglia in a partially immature phenotype(Erny et al., 2015), we decided to compare brains upon withdrawal of the drug with brains which were never exposed to doxycycline as a control, in order to avoid the overestimation of microglia response. Mice were housed in groups of three to five on a standard $12 \mathrm{~h}$ light/12 h dark cycle, and all experiments were performed during the light cycle. Food and water were provided ad libitum.

Brain samples and analyzed brain regions. We analyzed brains of both sexes from C57BL/6J adult mice (8-12 weeks); Cx3cr1 ${ }^{\mathrm{GFP} /-}$ mice at postnatal time points P7, P15, P21; 5xFAD mice after 3 and 6 months; and CK-p25 mice 1, 2, and 6 weeks after doxycycline withdrawal. We focused on the following brain regions: the glomerular layer of the olfactory bulb (OB), cortical layer III-V of the frontal cortex (FC) and the primary somatosensory cortex (S1), the dentate gyrus of the hippocampus (DG), the substantia nigra (SN), the cochlear nucleus $(\mathrm{CN})$, and the third lobe of the cerebellum (CB). The sagittal view of the brain sections analyzed (Fig. 1A, 2A, 3A, 4A, 5A and Supp. Fig. 4A and 5A) was taken from the Allen Developing Mouse Brain Atlas-Sagittal atlas and modified to show brain regions-of-interest (Lein et al., 2006).

Ovariectomy. Adolescent C57BL/6J females at P20 were anesthetized with 5\% isoflurane in $0.5 \mathrm{l} / \mathrm{min} \mathrm{O}_{2}$ during the anesthesia induction and $2 \%$ isoflurane in $0.5 \mathrm{l} / \mathrm{min} \mathrm{O}_{2}$ during the maintenance phase. Using an electric razor, the fur was shaved to expose the skin over the lumbar spine and the region was sterilized with $70 \%(\mathrm{v} / \mathrm{v})$ ethanol. A midline incision of 
approximately $1 \mathrm{~cm}$ was made on the skin in the lower back, below the chest. The subcutaneous tissue was gently dissected to expose the muscular fascia, and the ovarian fatpad was identified under the muscular layer. The peritoneal cavity was cut with a $0.5 \mathrm{~cm}$ incision. The Fallopian tube was exposed, and the ovary identified and cut at the level of the oviduct. The blood vessels were cauterized to prevent bleeding. The remaining part of the Fallopian tube was placed back in the peritoneal cavity, and the muscular fascia was sutured. The same protocol was repeated for the contralateral ovary. At the end, the skin was sutured. The animals received Metamizol (Sanofi Aventis, Cat\#Ay005, s.c. 200 mg/kg during surgery) and Meloxicam (Boehringer-Ingelheim, Cat\#KPOEH3R, s.c. $5 \mathrm{mg} / \mathrm{kg}$ after surgery every $24 \mathrm{~h}$ for 3 consecutive days), s.c., $2 \mathrm{mg} / \mathrm{kg}$ after surgery. Animals were sacrificed at P60.

Transcardiac perfusion. For histological analysis, animals were quickly anesthetized with isoflurane (Zoetis, Cat\#6089373) and secured to the perfusion plate as described previously (Venturino et al., 2021). In short: animals were initially perfused with $30 \mathrm{ml}$ of phosphatebuffered saline (PBS) with heparin (100 mg/l, Sigma, Cat\#H0878), followed by $30 \mathrm{ml}$ of 4\% (w/v) paraformaldehyde (PFA, Sigma, Cat\#P6148) in PBS using a peristaltic pump (Behr, Cat\#PLP 380, speed: $25 \mathrm{rpm}$ ). Animals were decapitated, the brain explanted, fixed in 4\% (w/v) PFA for 30 minutes and post-fixed in 4\% (w/v) PBS overnight (16h). Then the tissues were washed in PBS and stored at $4^{\circ} \mathrm{C}$ with $0.025 \%$ (w/v) sodium azide (VWR, Cat\#786299). For cryoprotection, the tissue was transferred to $30 \%$ (w/v) sucrose (Sigma, Cat\#84097) in PBS and incubated overnight at $4{ }^{\circ} \mathrm{C}$. To increase antibody permeability, the brain slices were frozen over dry-ice and thawed at room temperature for three cycles.

Vibratome sections. Cryoprotected samples were embedded into 3\% (w/v) Agarose/PBS to obtain coronal brain sections. The brain was sliced in $100 \mu \mathrm{m}$ coronal sections on a vibratome (Leica VT 1200S).

Immunofluorescence staining. The brain slices were incubated in blocking solution containing 1\% (w/v) bovine serum albumin (Sigma, Cat\#A9418), 5\% (v/v) Triton X-100 (Sigma, Cat\#T8787), 0.5\% (w/v) sodium azide (VWR, Cat\#786-299), and 10\% (v/v) serum (either goat, Millipore, Cat\#S26, or donkey, Millipore, Cat\#S30) for 1 hour at room temperature on a shaker. Afterwards, the samples were immunostained with primary antibodies diluted in antibody solution containing $1 \%(\mathrm{w} / \mathrm{v})$ bovine serum albumin, $5 \%(\mathrm{v} / \mathrm{v})$ triton X-100, $0.5 \%(\mathrm{v} / \mathrm{v})$ sodium azide, $3 \%(\mathrm{v} / \mathrm{v})$ goat or donkey serum, and incubated for 48 
hours on a shaker at room temperature. The following primary antibodies were used: rat $\alpha$ CD68 (AbD Serotec, Cat\#MCA1957, clone FA-11, Lot 1807, 1:250); goat $\alpha$-Iba1 (Abcam, ab5076, Lot FR3288145-1, 1:250); rabbit anti-Iba1 (GeneTex, Cat\#GTX100042, Lot 41556, 1:750). The slices were then washed 3 times with PBS and incubated protected from light for 2 hours at room temperature on a shaker, with the secondary antibodies diluted in antibody solution. The secondary antibodies raised in goat or donkey were purchased from Thermo Fisher Scientific (Alexa Fluor 488, Alexa Fluor 568, Alexa Fluor 647, 1:2000). The slices were washed 3 times with PBS. The nuclei were labeled with Hoechst 33342 (Thermo Fisher Scientific, Cat\#H3570, 1:5000) diluted in PBS for 15 minutes. The slices were mounted on microscope glass slides (Assistant, Cat\#42406020) with coverslips (Menzel-Glaser \#0) using an antifade solution [10\% (v/v) mowiol (Sigma, Cat\#81381), 26\% (v/v) glycerol (Sigma, Cat\#G7757), 0.2M tris buffer $\mathrm{pH} 8,2.5 \%$ (w/v) Dabco (Sigma, Cat\#D27802)].

Confocal microscopy. Images were acquired with a Zeiss LSM880 upright Airy scan or with a Zeiss LSM700 upright using a Plan-Apochromat $40 \times$ oil immersion objective N.A. 1.4. $2 \times$ 2 z-stack tail-images were acquired with a resolution of $1024 \times 1024$ pixels.

Image processing. Confocal tile images were stitched using the software Imaris Stitcher 9.3.1.v. Then, the confocal images were loaded in Fiji 1.52e (http://imagej.net/Fiji). To remove the background, the rolling ball radius was set to 35 pixels, and images were filtered using a median 3D filter with $\mathrm{x}, \mathrm{y}, \mathrm{z}$ radii set at 3. Image stacks were exported as tif files, converted to .ims files using the Imaris converter, and imported into Imaris 8.4.2.v. (Bitplane Imaris).

Quantification of CD68 volume within cells. Surface renderings were generated on microglia and CD68 z-stacks using the surface-rendering module of Imaris 9.2.v Surfaces were generated with the surface detail set to $0.2 \mu \mathrm{m}$. To determine the CD68 surface within microglia, the surface-surface coloc plugin was used. This analysis was performed on the entire image. The total ratio of CD68 volume within microglial volume (CD68-to-microglial volume) was calculated per image. To compute the CD68 fold change, the total CD68-tomicroglial volume from each condition (sex/time-point) was scaled to the CD68-to-microglial volume ratio from the respective controls. CD68 fold change $>1$ means an increase in CD68 volume, $\mathrm{CD} 68<1$ means a decrease in CD68 volume. CD68 fold-change $=1$ denotes no change in CD68 volume. 
Quantification of microglia density and statistical analysis. The spot-function plugin of Imaris 9.2.v was used to count the number of cells, i.e the soma of iba-1 positive microglia within every confocal image. Microglial cell density was estimated as total number of cells obtained in this way, divided by the size of the imaged sample in $\mathrm{mm}^{2}$. Sex averages for microglia from each region were compared with two-sided t.test.

Reconstruction of 3D-traced microglia. After filtering and background subtraction, images were imported in Imaris 9.2.v (Bitplane Imaris). Microglial processes were 3D-traced with the filament-tracing plugin. Since the filament-tracing plugin provides a semi-automated reconstruction, this eliminates the need for a user-blind approach for selecting representative microglia. New starting points were detected when the largest diameter was set to $12 \mu \mathrm{m}$ and with seeding points of $1 \mu \mathrm{m}$. Disconnected segments were removed with a filtering smoothness of $0.6 \mu \mathrm{m}$. After the tracing, we manually removed cells that were sitting at the border of the image and were only partially traced so that these cells would not be analyzed. The generated skeleton images were converted from .ims format (Imaris) to .swc format ( Stockley, E. W., et al., 1993) by first obtaining the 3D positions $(x, y, z)$ and the diameter of each traced microglial process using the ImarisReader toolbox for MATLAB (https://github.com/PeterBeemiller/ImarisReader) and then exporting for format standardization using the NL Morphology Converter (http://neuroland.org).

Analysis of morphometric features. Classic morphometric features were calculated from the .swc files using the functions Length (for total process length), $\mathrm{N}$ branch (for number of branches), N_bifs (for number of branching points) and N_tips (for number of terminal points) from L-measure (Scorcioni et al., 2008) (http://cng.gmu.edu:8080/Lm/). Statistical analysis was performed using scipy.stats (v1.6.2) and scikit-posthocs (v0.6.7). These morphometric features were first tested for normality using the Kolmogorov-Smirnov test (scipy.stats.kstest). After determining the non-normal distribution of the features, we performed non-parametric pairwise tests for independence between measurements from two brain regions using the Kruskal-Wallis test (scipy.stats.kruskal). We used Bonferronicorrected P values, calculated using Dunn's test via scikit_posthocs.posthoc_dunn (Supp. Table 2-3).

Sholl analysis. Sholl curves were calculated from the .swc files using the sholl_crossings function of the NeuroM Python toolkit (https://github.com/BlueBrain/NeuroM). In brief, concentric Sholl spheres centered on the soma of a given traced microglia are constructed 
with a given step size radius. The number of microglial processes that intersect each Sholl sphere are determined. This step is performed for each traced microglia in the data. From this, Sholl curves of a microglial population are then calculated as the average number of intersections across the population.

Topological morphology descriptor (TMD). A topological data analysis algorithm, the TMD, was used to extract topological phenotypes, called persistence barcodes, from 3D morphological structures (https://github.com/BlueBrain/TMD, Kanari et. al., 2018). In brief, the $3 \mathrm{D}$ reconstructed microglia is represented as a tree $T$ rooted in its soma. The TMD summarizes this tree by calculating persistence barcodes, where each bar represents a persistent microglial process with respect to a filtering function, i.e., the radial distance from the soma. Note that the persistence barcode that the TMD associates with $T$ under this filtering function is invariant under rotations about the root and rigid translations of $T$ in $R^{3}$.

Each bar is described by two numbers: the radial distance, $d_{i}$, at which a process originates; and the distance, $b_{i}$, when it merges with a larger, more persistent process or with the soma. A bar can be equivalently represented as a point $\left(d_{i}, b_{i}\right)$ in a persistence diagram. We could therefore convolve each point in the persistence diagram with a Gaussian kernel and discretize it to generate a matrix of pixel values, encoding the persistence diagram in a vector, called the persistence image.

Average and bootstrapped persistence images. To construct the average persistence image of a given condition, all the persistence barcodes of microglia from the same condition are combined before Gaussian convolution and discretization are performed. We also constructed average persistence images by performing first the Gaussian convolution and discretization of individual microglia persistence barcodes before taking the pixel-wise average. This produced qualitatively similar results.

The bootstrapping method subsamples the microglial population within a given condition, thereby introducing variations around the average persistence image. Starting from the population of all microglia from the same condition, called the starting population of size $n$, the persistence barcodes of a pre-defined number of unique microglia, called the bootstrap size, are combined to calculate the bootstrapped persistence image. We iterate this process a pre-defined number of times, $n_{\text {samples }}$, with replacement to obtain the bootstrap sample. 
Subtraction images and TMD distance. The subtraction image is the pixel-wise difference between two given persistence images. From the subtraction image, the TMD distance can be computed as the sum of the absolute pixel-wise difference. For stability of the TMD distance, we refer the reader to Kanari et al. (Kanari et al., 2017)

Hierarchical clustering. Hierarchical clustering allowed us to find similarities between microglia across several conditions. Hierarchical clustering was done on the basis of the average persistence images. Clusters were then identified hierarchically using the average linkage criterion with the TMD distance metric and was implemented using cluster.hierarchy.linkage from SciPy v1.6.2 (https://www.scipy.org). Dendrograms were generated using cluster.hierarchy.dendrogram to visualize the arrangement of the resulting cluster.

\section{Dimensionality reduction.}

UMAP. A fast, non-linear dimensionality reduction algorithm, UMAP (Mcinnes et al., 2020) (Uniform Manifold Approximation and Projection), was applied to visualize the highdimensional pixel space of bootstrapped persistence images using a 2D representation while preserving local and global structures in the bootstrap samples (https://github.com/lmcinnes/umap)(Mcinnes et al., 2020). Given a bootstrap sample containing multiple conditions, a TMD distance matrix containing pairwise distances between bootstrapped persistence images in the bootstrap sample is calculated. Principal components are then obtained using a singular value decomposition of the TMD distance matrix. The first 7 principal components, where the elbow in the singular values is located, were used as input to UMAP with $n \_$neighbors $=50$, min_dist $=1.0$ and spread $=3.0$. Note that we have tested for a wide range of parameter values which did not qualitatively change any of the observations we made in the main text (Supp. Fig. 2E).

$t S N E$. An alternative dimensionality reduction algorithm is $\mathrm{tSNE}^{83}$ (t-distributed Stochastic Neighbor Embedding, https://github.com/DmitryUlyanov/Multicore-TSNE) which finds a dimensionality-reduced representation where similar points are pulled closer together while dissimilar points are pushed farther apart with high probability. The first 7 principal components were taken as an input to run tSNE with perplexity $=50$.

Pseudotemporal ordering. The concept of morphological phenotypes as encoded in the persistence images can be likened to transcriptional phenotypes in single-cell RNA 
sequencing studies. Bootstrapped persistence images, which encapsulate morphological phenotypes of microglial populations from similar conditions, are comparable. Furthermore, it is reasonable to assume that morphological changes in bootstrapped microglial populations from control to disease conditions occur with incremental differences in the persistence images. This conceptual similarity allowed us to use the pseudo-temporal trajectory inference algorithms that are well-used in the single-cell RNA sequencing community to study the morphological progression during microglial development and degeneration.

Palantir. Palantir (Setty et al., 2019) uses principles from graph theory and Markov processes to calculate the pseudo-time and the probability of a cell reaching each of the terminal conditions in the sample (https://github.com/dpeerlab/Palantir). First, the principal components of the bootstrapped persistence images were obtained using palantir.utils.run_pca with n_components $=100$ and use_hvg $=$ False. The diffusion maps were then calculated from the PCA projections using palantir.utils.run_diffusion_maps with n_components $=10$ and $\mathrm{knn}=20$ which outputs the Palantir pseudo-times. Harmony (Nowotschin et al., 2019) is then used to construct an augmented affinity matrix from the Palantir pseudo-times to connect together the Palantir pseudo-times and construct a trajectory using a force-directed graph (https://github.com/dpeerlab/Harmony).

Monocle. To corroborate the Palantir trajectories, an alternative pseudo-temporal trajectoryinference algorithm called Monocle was employed. Monocle (Cao et al., 2019) uses reverse graph embedding which learns a principal graph which approximates a lower-dimensional manifold to construct a pseudo-time trajectory (https://github.com/cole-trapnell$\underline{\text { lab/monocle3 }}$ (Cao et al., 2019). Similar to Palantir implementation, the principal components of the bootstrapped persistence images were first obtained using preprocess_cds with num_dim $=100$. A 2D UMAP representation was then obtained using reduce_dimension with umap.metric $=$ "manhattan", umap.min_dist $=1.0$, and clusters were identified using cluster_cells with cluster_method = 'leiden'. Finally, the pseudo-temporal trajectory was then obtained using learn_graph with use_partition = FALSE and close_loop = FALSE.

Stable ranks analysis. An alternative representation of the persistence barcodes is through stable ranks (Riihimäki and Chacholski, 2018). Stable ranks are functional summaries of persistence which depend on pseudometrics to compare persistence barcodes. Given a 
pseudometric $d$, the stable rank $\operatorname{rank}_{d}(X)(t)$ of a persistence barcode $X$ is a function that assigns to $t$ the number:

$$
\operatorname{rank}_{d}(X)(t)=\min \{\operatorname{rank}(Y) \mid d(Y, X) \leq t\}
$$

whereby $\operatorname{rank}(Y)$ denotes the number of bars of the persistence barcode $Y$. The stable rank $r \hat{a n n} k_{d}(X)(t)$ associates to a persistence barcode a non-increasing and piece-wise constant function with values in $[0, \infty)$. An important property is that this mapping is continuous with respect to the chosen pseudometric $d$ and the $L_{p}$ metric on the space $\mathscr{M}$ of measurable functions.

A class of pseudometrics on persistence barcodes can be constructed from density functions (Riihimäki and Chacholski, 2018), which intuitively are used to vary the weight along the filtration scale parametrizing a barcode. With such pseudometrics, the stable rank is a bar count based on length of bars as scaled by the density. The standard stable rank is defined by a density function with constant value one. In this case, $r a \hat{n} k_{d}(X)(t)$ is the number of bars in $X$ with length greater than or equal to $t$, i.e., all filtration scales are weighted equally.

Stable ranks can be used in place of persistence images in the MorphOMICs pipeline. Similarly, to MorphOMICs, the persistence barcode $X$ of a given microglia is calculated using the TMD algorithm. To obtain bootstrapped standard stable ranks, we combined the persistence barcodes of a pre-defined number of microglia and computed their standard stable ranks. Dimensionality reduction was then implemented similar to the methods above (see

\section{Methods: Dimensionality reduction).}

Classification accuracy using stable ranks. To support and quantify the impact of bootstrapping on the regional segregation visualized in the reduced UMAP space (Fig. 1F), we performed a classification task for microglia morphologies represented by their standard stable rank and labeled by brain region. We used a support vector machine (SVM) with a specific kernel based on stable ranks (Agerberg et al., 2021) for the classification. For persistence barcodes $X$ and $Y$, the stable rank kernel with respect to a pseudometric $d$ is given by

$$
K_{d}(X, Y):=\int_{0}^{\infty} \operatorname{rank}_{d}(X)(t) \operatorname{rank}_{d}(Y)(t) d t
$$

where we used the pseudometric induced by the constant function with value one. 
We performed pairwise classifications. For each pair of brain regions, we constructed a dataset consisting of 400 bootstrap samples, i.e., 200 from each region and bootstrap sizes of either 10, 20 or 50 (the results are reported separately for these three values). We randomly partition the dataset for cross-validation wherein 240 samples were used for SVM training (training set) and 160 samples for validation (test set). We report the average accuracy over 10 repeated cross-validations on the test set. The SVM was trained using the implementation in the Python library sklearn (https://scikit-learn.org/stable/) with default settings except for the usage of the stable rank kernel.

Bootstrapped morphometric features and bootstrapped Sholl curves. To understand whether classical morphology analysis pipelines are able to recapitulate the microglial dynamics recovered by MorphOMICs, a similar bootstrapping analysis was also done where we pooled together a pre-defined number of microglia. Each morphometric quantity in the extended list enumerated in Supp. Table 4 was then averaged to obtain a 27-D vector, with each dimension corresponds to a morphometric feature, called the bootstrapped morphometric features. On the other hand, Sholl curves averaged across the pooled microglia to obtain the bootstrapped Sholl curves. Dimensionality reduction was then implemented similar to the methods above (see Methods: Dimensionality reduction).

Data availability. All the .swc files are available upon request.

\section{$\underline{\text { Acknowledgements }}$}

We thank the scientific service units at IST Austria, in particular Michael Schunn's team at the preclinical facility, and especially our colony manager Sonja Haslinger, for excellent support. We are also grateful to the IST bioimaging facility, and in particular Christoph Sommer for helping with the data file conversions. We thank Margaret Maes, Balint Nagy, Sara Oakeley, Marco Benevento and all members of the Siegert group for constant feedback on the project and on the manuscript. This research was supported by the European Union Horizon 2020 research and innovation program under the Marie Skłodowska-Curie Actions program (754411 to R.J.A.C.), and by the European Research Council (grant No. 715571 to S.S.). L.K. was supported by funding to the Blue Brain Project, a research center of the École polytechnique fédérale de Lausanne, from the Swiss government's ETH Board of the Swiss Federal Institutes of Technology. 


\section{$\underline{\text { Author contributions }}$}

Conceptualization, G.C., R.J.A.C, L.K., M.S., J.A., W.C., K.H., S.S.; Methodology, G.C., R.J.A.C., L.K., S.S.; Software, R.J.A.C., L.K.; Validation, G.C., R.J.A.C.; Formal analysis, G.C., R.J.A.C., L.K.; Investigation, G.C., R.J.A.C., A.V., R.S., S.S.; Resources, L.K., H.M., L-H.T.; Data Curation, G.C., R.J.A.C.; Writing - Original Draft and Visualization, G.C., R.J.A.C., S.S.; Supervision, Project Administration, Funding Acquisition, S.S.; Stable ranks: Conceptualization, G.C., R.J.A.C., L.K., M.S., J.A., W.C., K.H., S.S.; Software Validation, and Formal Analysis, R.J.A.C., J.A.

\section{$\underline{\text { Declaration of interests }}$}

The authors declare no competing financial or non-financial interests.

\section{$\underline{\text { References }}$}

Adams, H., Emerson, T., Kirby, M., Neville, R., Peterson, C., Shipman, P., Hanson, E., Motta, F., and Ziegelmeier, L. (2017). Persistence Images: A Stable Vector Representation of Persistent Homology. J. Mach. Learn. Res. 18, 1-35.

Adeluyi, A., Guerin, L., Fisher, M.L., Galloway, A., Cole, R.D., Chan, S.S.L., Wyatt, M.D., Davis, S.W., Freeman, L.R., Ortinski, P.I., et al. (2019). Microglia morphology and proinflammatory signaling in the nucleus accumbens during nicotine withdrawal. Sci. Adv. 5.

Agerberg, J., Ramanujam, R., Scolamiero, M., and Chachólski, W. (2021). Supervised Learning Using Homology Stable Rank Kernels. Front. Appl. Math. Stat. 0, 39.

Ayata, P., Badimon, A., Strasburger, H.J., Duff, M.K., Montgomery, S.E., Loh, Y.H.E., Ebert, A., Pimenova, A.A., Ramirez, B.R., Chan, A.T., et al. (2018). Epigenetic regulation of brain region-specific microglia clearance activity. Nat. Neurosci. 21, 1049-1060.

Bachstetter, A.D., Van Eldik, L.J., Schmitt, F.A., Neltner, J.H., Ighodaro, E.T., Webster, S.J., Patel, E., Abner, E.L., Kryscio, R.J., and Nelson, P.T. (2015). Disease-related microglia heterogeneity in the hippocampus of Alzheimer's disease, dementia with Lewy bodies, and 
hippocampal sclerosis of aging. Acta Neuropathol. Commun.

Bakkour, A., Morris, J.C., Wolk, D.A., and Dickerson, B.C. (2013). The effects of aging and Alzheimer's disease on cerebral cortical anatomy: Specificity and differential relationships with cognition. Neuroimage 76, 332-344.

Ben-Ari, Y. (2002). Excitatory actions of GABA during development: The nature of the nurture. Nat. Rev. Neurosci. 3, 728-739.

Bijari, K., Valera, G., López-Schier, H., and Ascoli, G.A. (2021). Quantitative neuronal morphometry by supervised and unsupervised learning. STAR Protoc. 2, 100867.

Braak, H., Braak, E., Bohl, J., and Lang, W. (1989). Alzheimer's disease: amyloid plaques in the cerebellum. J. Neurol. Sci. 93, 277-287.

Brar, S., Henderson, D., Schenck, J., and Zimmerman, E.A. (2009). Iron Accumulation in the Substantia Nigra of Patients With Alzheimer Disease and Parkinsonism. Arch. Neurol. 66, $371-374$.

Bruce-Keller, A.J., Keeling, J.L., Keller, J.N., Huang, F.F., Camondola, S., and Mattson, M.P. (2000). Antiinflammatory effects of estrogen on microglial activation. Endocrinology $141,3646-3656$.

Burns, J.M., Galvin, J.E., Roe, C.M., Morris, J.C., and McKeel, D.W. (2005). The pathology of the substantia nigra in Alzheimer disease with extrapyramidal signs. Neurology 64, 13971403.

Caligioni, C.S. (2009). Assessing reproductive status/stages in mice. Curr. Protoc. Neurosci. APPENDIX, Appendix.

Camins, A., Verdaguer, E., Folch, J., Canudas, A.M., and Pallàs, M. (2006). The role of CDK5/P25 formation/inhibition in neurodegeneration. Drug News Perspect. 19, 453-460.

Cao, J., Spielmann, M., Qiu, X., Huang, X., Ibrahim, D.M., Hill, A.J., Zhang, F., Mundlos, S., Christiansen, L., Steemers, F.J., et al. (2019). The single-cell transcriptional landscape of mammalian organogenesis. Nat. 20195667745 566, 496-502.

Carlsson, G. (2009). TOPOLOGY AND DATA. 
Chistiakov, D.A., Killingsworth, M.C., Myasoedova, V.A., Orekhov, A.N., and Bobryshev, Y. V. (2017). CD68/macrosialin: Not just a histochemical marker. Lab. Investig. 97, 4-13.

Congdon, E.E. (2018). Sex differences in autophagy contribute to female vulnerability in Alzheimer's disease. Front. Neurosci. 12, 372.

Crain, J.M., and Watters, J.J. (2010). Estrogen and P2 purinergic receptor systems in microglia: Therapeutic targets for neuroprotection. Open Drug Discov. J. 2, 148-167.

Cruz, J.C., and Tsai, L.H. (2004). A Jekyll and Hyde kinase: Roles for Cdk5 in brain development and disease. Curr. Opin. Neurobiol. 14, 390-394.

Cruz, J.C., Tseng, H.C., Goldman, J.A., Shih, H., and Tsai, L.H. (2003). Aberrant Cdk5 activation by p 25 triggers pathological events leading to neurodegeneration and neurofibrillary tangles. Neuron 40, 471-483.

Cruz, J.C., Kim, D., Moy, L.Y., Dobbin, M.M., Sun, X., Bronson, R.T., and Tsai, L.H. (2006). p25/cyclin-dependent kinase 5 induces production and intraneuronal accumulation of amyloid $\beta$ in vivo. J. Neurosci. 26, 10536-10541.

Davalos, D., Lee, J.K., Smith, W.B., Brinkman, B., Ellisman, M.H., Zheng, B., and Akassoglou, K. (2008). Stable in vivo imaging of densely populated glia, axons and blood vessels in the mouse spinal cord using two-photon microscopy. J. Neurosci. Methods 169, 17.

DeKosky, S.T., and Scheff, S.W. (1990). Synapse loss in frontal cortex biopsies in Alzheimer's disease: Correlation with cognitive severity. Ann. Neurol. 27, 457-464.

Desikan, R.S., Sabuncu, M.R., Schmansky, N.J., Reuter, M., Cabral, H.J., Hess, C.P., Weiner, M.W., Biffi, A., Anderson, C.D., Rosand, J., et al. (2010). Selective Disruption of the Cerebral Neocortex in Alzheimer's Disease. PLoS One 5, e12853.

Erny, D., De Angelis, A.L.H., Jaitin, D., Wieghofer, P., Staszewski, O., David, E., KerenShaul, H., Mahlakoiv, T., Jakobshagen, K., Buch, T., et al. (2015). Host microbiota constantly control maturation and function of microglia in the CNS. Nat. Neurosci. 2015187 $18,965-977$.

Fischer, A., Sananbenesi, F., Pang, P.T., Lu, B., and Tsai, L.H. (2005). Opposing roles of 
transient and prolonged expression of p25 in synaptic plasticity and hippocampus-dependent memory. Neuron 48, 825-838.

Furube, E., Kawai, S., Inagaki, H., Takagi, S., and Miyata, S. (2018). Brain Regiondependent Heterogeneity and Dose-dependent Difference in Transient Microglia Population Increase during Lipopolysaccharide-induced Inflammation. Sci. Rep. 8.

Gamache, J., Yun, Y., and Chiba-Falek, O. (2020). Sex-dependent effect of APOE on Alzheimer's disease and other age-related neurodegenerative disorders. Dis. Model. Mech. 13.

Ginhoux, F., Greter, M., Leboeuf, M., Nandi, S., See, P., Gokhan, S., Mehler, M.F., Conway, S.J., Ng, L.G., Stanley, E.R., et al. (2010). Fate mapping analysis reveals that adult microglia derive from primitive macrophages. Science 330, 841-845.

Ginhoux, F., Lim, S., Hoeffel, G., Low, D., and Huber, T. (2013). Origin and differentiation of microglia. Front. Cell. Neurosci. 0, 45.

González-Scarano, F., and Baltuch, G. (1999). MICROGLIA AS MEDIATORS OF

INFLAMMATORY AND DEGENERATIVE DISEASES. Annu. Rev. Neurosci. 22, 219240.

Gosche, K.M., Mortimer, J.A., Smith, C.D., Markesbery, W.R., and Snowdon, D.A. (2002). Hippocampal volume as an index of Alzheimer neuropathology. Neurology 58, 1476-1482.

Gouwens, N.W., Sorensen, S.A., Berg, J., Lee, C., Jarsky, T., Ting, J., Sunkin, S.M., Feng, D., Anastassiou, C.A., Barkan, E., et al. (2019). Classification of electrophysiological and morphological neuron types in the mouse visual cortex. Nat. Neurosci. 2019227 22, $1182-$ 1195.

Grabert, K., and McColl, B.W. (2018). Isolation and phenotyping of adult mouse microglial cells. In Methods in Molecular Biology, (Humana Press Inc.), pp. 77-86.

Greter, M., and Merad, M. (2013). Regulation of microglia development and homeostasis. Glia $61,121-127$.

Han, J., Fan, Y., Zhou, K., Blomgren, K., and Harris, R.A. (2021). Uncovering sex differences of rodent microglia. J. Neuroinflammation 2021181 18, 1-11. 
Hattori, T., and McGeer, P.L. (1973). Synaptogenesis in the corpus striatum of infant rat. Exp. Neurol. 38, 70-79.

Heindl, S., Gesierich, B., Benakis, C., Llovera, G., Duering, M., and Liesz, A. (2018). Automated morphological analysis of microglia after stroke. Front. Cell. Neurosci. 12.

Hemonnot, A.L., Hua, J., Ulmann, L., and Hirbec, H. (2019). Microglia in Alzheimer disease: Well-known targets and new opportunities. Front. Cell. Infect. Microbiol. 9, 233.

Ito, D., Imai, Y., Ohsawa, K., Nakajima, K., Fukuuchi, Y., and Kohsaka, S. (1998).

Microglia-specific localisation of a novel calcium binding protein, Iba1. Mol. Brain Res. 57, $1-9$.

Jacobs, H.I.L., Hopkins, D.A., Mayrhofer, H.C., Bruner, E., Van Leeuwen, F.W., Raaijmakers, W., and Schmahmann, J.D. (2018). The cerebellum in Alzheimer's disease: evaluating its role in cognitive decline. Brain 141, 37-47.

Jantzie, L.L., Cheung, P.Y., and Todd, K.G. (2005). Doxycycline reduces cleaved caspase-3 and microglial activation in an animal model of neonatal hypoxia-ischemia. J. Cereb. Blood Flow Metab. 25, 314-324.

Jiang, X., Shen, S., Cadwell, C.R., Berens, P., Sinz, F., Ecker, A.S., Patel, S., and Tolias, A.S. (2015). Principles of connectivity among morphologically defined cell types in adult neocortex. Science (80-. ). 350.

Kanari, L., Dłotko, P., Scolamiero, M., Levi, R., Shillcock, J., Hess, K., and Markram, H. (2017). A Topological Representation of Branching Neuronal Morphologies.

Neuroinformatics 2017161 16, 3-13.

Kanari, L., Dictus, H., Chalimourda, A., Geit, W. Van, Coste, B., Shillcock, J., Hess, K., and Markram, H. (2020). Computational synthesis of cortical dendritic morphologies. BioRxiv 2020.04.15.040410.

Kazee, A.M., Cox, C., and Richfield, E.K. (1995). Substantia nigra lesions in Alzheimer disease and normal aging. Alzheimer Dis. Assoc. Disord. 9, 61-67.

Kongsui, R., Beynon, S.B., Johnson, S.J., and Walker, F.R. (2014). Quantitative assessment of microglial morphology and density reveals remarkable consistency in the distribution and 
morphology of cells within the healthy prefrontal cortex of the rat. J. Neuroinflammation 11, $1-9$.

Kovács, T., Cairns, N.J., and Lantos, P.L. (1999). beta-amyloid deposition and neurofibrillary tangle formation in the olfactory bulb in ageing and Alzheimer's disease. Neuropathol. Appl. Neurobiol. 25, 481-491.

Kozlowski, C., and Weimer, R.M. (2012). An automated method to quantify microglia morphology and application to monitor activation state longitudinally in vivo. PLoS One 7.

Kroon, T., van Hugte, E., van Linge, L., Mansvelder, H.D., and Meredith, R.M. (2019). Early postnatal development of pyramidal neurons across layers of the mouse medial prefrontal cortex. Sci. Rep. 9, 1-16.

Larner, A.J. (1997). The Cerebellum in Alzheimer's Disease. Dement. Geriatr. Cogn. Disord. 8, 203-209.

Lawson, L.J., Perry, V.H., Dri, P., and Gordon, S. (1990). Heterogeneity in the distribution and morphology of microglia in the normal adult mouse brain. Neuroscience 39, 151-170.

Lehtovirta, M., Laakso, M.P., Soininen, H., Helisalmi, S., Mannermaa, A., Helkala, E.L., Partanen, K., Ryynänen, M., Vainio, P., Hartikainen, P., et al. (1995). Volumes of hippocampus, amygdala and frontal lobe in Alzheimer patients with different apolipoprotein E genotypes. Neuroscience 67, 65-72.

Lein, E.S., Hawrylycz, M.J., Ao, N., Ayres, M., Bensinger, A., Bernard, A., Boe, A.F., Boguski, M.S., Brockway, K.S., Byrnes, E.J., et al. (2006). Genome-wide atlas of gene expression in the adult mouse brain. Nat. 20064457124 445, 168-176.

Lenz, K.M., and McCarthy, M.M. (2015). A starring role for microglia in brain sex differences. Neuroscientist 21, 306-321.

Lenz, K.M., Nugent, B.M., Haliyur, R., and McCarthy, M.M. (2013). Microglia are essential to masculinization of brain and behavior. J. Neurosci. 33, 2761-2772.

Leuba, G., Savioz, A., Vernay, A., Carnal, B., Kraftsik, R., Tardif, E., Riederer, I., and Riederer, B.M. (2008). Differential Changes in Synaptic Proteins in the Alzheimer Frontal Cortex with Marked Increase in PSD-95 Postsynaptic Protein. J. Alzheimer's Dis. 15, 139- 
151.

Li, Y., Wang, D., Ascoli, G.A., Mitra, P., and Wang, Y. (2017). Metrics for comparing neuronal tree shapes based on persistent homology. PLoS One 12, e0182184.

Van Der Maaten, L., and Hinton, G. (2008). Visualizing Data using t-SNE. J. Mach. Learn. Res. 9, 2579-2605.

Maklad, A., and Fritzsch, B. (2003). Development of vestibular afferent projections into the hindbrain and their central targets. Brain Res. Bull. 60, 497-510.

Manji, Z., Rojas, A., Wang, W., Dingledine, R., Varvel, N.H., and Ganesh, T. (2019). 5xFAD Mice Display Sex-Dependent Inflammatory Gene Induction During the Prodromal Stage of Alzheimer's Disease. J. Alzheimer's Dis. 70, 1259-1274.

Mcinnes, L., Healy, J., and Melville, J. (2020). UMAP: Uniform Manifold Approximation and Projection for Dimension Reduction.

Mildner, A., Schlevogt, B., Kierdorf, K., Böttcher, C., Erny, D., Kummer, M.P., Quinn, M., Brück, W., Bechmann, I., Heneka, M.T., et al. (2011). Distinct and non-redundant roles of microglia and myeloid subsets in mouse models of Alzheimer's disease. J. Neurosci. 31, 11159-11171.

Morrison, H.W., and Filosa, J.A. (2013). A quantitative spatiotemporal analysis of microglia morphology during ischemic stroke and reperfusion. J. Neuroinflammation 10, 4.

Morrison, H., Young, K., Qureshi, M., Rowe, R.K., and Lifshitz, J. (2017). Quantitative microglia analyses reveal diverse morphologic responses in the rat cortex after diffuse brain injury. Sci. Rep. 7, 1-12.

Moschovakis, A.K., Karabelas, A.B., and Highstein, S.M. (1988). Structure-function relationships in the primate superior colliculus. I. Morphological classification of efferent neurons. Https://Doi.Org/10.1152/Jn.1988.60.1.232 60, 232-262.

Nelson, L.H., Warden, S., and Lenz, K.M. (2017). Sex differences in microglial phagocytosis in the neonatal hippocampus. Brain. Behav. Immun. 64, 11-22.

Nimmerjahn, A., Kirchhoff, F., and Helmchen, F. (2005). Resting Microglial Cells Are 
Highly Dynamic Surveillants of Brain Parenchyma in Vivo. Science (80-. ). 308, 1314-1318.

Nissen, J.C. (2017). Microglial function across the spectrum of age and gender. Int. J. Mol.

Sci. 18.

Nowotschin, S., Setty, M., Kuo, Y.-Y., Liu, V., Garg, V., Sharma, R., Simon, C.S., Saiz, N., Gardner, R., Boutet, S.C., et al. (2019). The emergent landscape of the mouse gut endoderm at single-cell resolution. Nat. 20195697756 569, 361-367.

Oakley, H., Cole, S.L., Logan, S., Maus, E., Shao, P., Craft, J., Guillozet-Bongaarts, A., Ohno, M., Disterhoft, J., Van Eldik, L., et al. (2006). Intraneuronal $\beta$-amyloid aggregates, neurodegeneration, and neuron loss in transgenic mice with five familial Alzheimer's disease mutations: Potential factors in amyloid plaque formation. J. Neurosci. 26, 10129-10140.

Ohm, T.G., and Braak, H. (1987). Olfactory bulb changes in Alzheimer's disease. Acta Neuropathol. 1987734 73, 365-369.

Ohm, T.G., and Braak, H. (1989). Auditory brainstem nuclei in Alzheimer's disease. Neurosci. Lett. 96, 60-63.

Paolicelli, R.C., Bolasco, G., Pagani, F., Maggi, L., Scianni, M., Panzanelli, P., Giustetto, M., Ferreira, T.A., Guiducci, E., Dumas, L., et al. (2011). Synaptic Pruning by Microglia Is Necessary for Normal Brain Development. Science (80-. ). 333, 1456-1458.

Payami, H., Zareparsi, S., Montee, K.R., Sexton, G.J., Kaye, J.A., Bird, T.D., Yu, C.E., Wijsman, E.M., Heston, L.L., Litt, M., et al. (1996). Gender difference in apolipoprotein E associated risk for familial alzheimer disease: A possible clue to the higher incidence of alzheimer disease in women. Am. J. Hum. Genet. 58, 803-811.

Perez-Pouchoulen, M., VanRyzin, J.W., and McCarthy, M.M. (2015). Morphological and phagocytic profile of microglia in the developing rat cerebellum. ENeuro 2, 36-51.

Polavaram, S., Gillette, T.A., Parekh, R., and Ascoli, G.A. (2014). Statistical analysis and data mining of digital reconstructions of dendritic morphologies. Front. Neuroanat. 8, 138.

Pont-Lezica, L., Béchade, C., Belarif-Cantaut, Y., Pascual, O., and Bessis, A. (2011).

Physiological roles of microglia during development. J. Neurochem. 119, 901-908. 
Rao, J.S., Keleshian, V.L., Klein, S., and Rapoport, S.I. (2012). Epigenetic modifications in frontal cortex from Alzheimer's disease and bipolar disorder patients. Transl. Psychiatry 2012272 , e132-e132.

Riihimäki, H., and Chacholski, W. (2018). Generalized persistence analysis based on stable rank invariant.

Rombaux, P., Huart, C., De Volder, A.G., Cuevas, I., Renier, L., Duprez, T., and Grandin, C. (2010). Increased olfactory bulb volume and olfactory function in early blind subjects.

Neuroreport 21, 1069-1073.

Ruusuvuori, E., Li, H., Huttu, K., Palva, J.M., Smirnov, S., Rivera, C., Kaila, K., and Voipio, J. (2004). Carbonic Anhydrase Isoform VII Acts as a Molecular Switch in the Development of Synchronous Gamma-Frequency Firing of Hippocampal CA1 Pyramidal Cells. J.

Neurosci. 24, 2699.

Santa-Cecília, F. V., Socias, B., Ouidja, M.O., Sepulveda-Diaz, J.E., Acuña, L., Silva, R.L., Michel, P.P., Del-Bel, E., Cunha, T.M., and Raisman-Vozari, R. (2016). Doxycycline Suppresses Microglial Activation by Inhibiting the p38 MAPK and NF-kB Signaling Pathways. Neurotox. Res. 29, 447-459.

Schwarz, J.M., Sholar, P.W., and Bilbo, S.D. (2012). Sex differences in microglial colonization of the developing rat brain. J. Neurochem. 120, 948-963.

Scorcioni, R., Polavaram, S., and Ascoli, G.A. (2008). L-Measure: a Web-accessible tool for the analysis, comparison, and search of digital reconstructions of neuronal morphologies. Nat. Protoc. 3, 866.

Serrano-Pozo, A., Frosch, M.P., Masliah, E., and Hyman, B.T. (2011). Neuropathological alterations in Alzheimer disease. Cold Spring Harb. Perspect. Med. 1, a006189.

Setty, M., Kiseliovas, V., Levine, J., Gayoso, A., Mazutis, L., and Pe'er, D. (2019). Characterization of cell fate probabilities in single-cell data with Palantir. Nat. Biotechnol. $37,451-460$.

Sholl, D.A. (1953). Dendritic organization in the neurons of the visual and motor cortices of the cat. J. Anat. 87, 387. 
Sinha, U.K., Hollen, K.M., Rodriguez, R., and Miller, C.A. (1993). Auditory system degeneration in Alzheimer's disease. Neurology 43, 779-779.

Stephen, J.M., Montaño, R., Donahue, C.H., Adair, J.C., Knoefel, J., Qualls, C., Hart, B., Ranken, D., and Aine, C.J. (2010). Somatosensory responses in normal aging, mild cognitive impairment, and Alzheimer's disease. J. Neural Transm. 117, 217-225.

Stockley, E. W., Cole, H. M., Brown, A. D., \& Wheal, H. V. (1993). A system for quantitative morphological measurement and electrotonic modelling of neurons: threedimensional reconstruction. Journal of neuroscience methods, 47(1-2), 39-51.

Stratoulias, V., Venero, J.L., Tremblay, M., and Joseph, B. (2019). Microglial subtypes: diversity within the microglial community. EMBO J. 38.

Struble, R.G., and Clark, H.B. (1992). Olfactory bulb lesions in alzheimer's disease. Neurobiol. Aging 13, 469-473.

Taipa, R., Ferreira, V., Brochado, P., Robinson, A., Reis, I., Marques, F., Mann, D.M., MeloPires, M., and Sousa, N. (2018). Inflammatory pathology markers (activated microglia and reactive astrocytes) in early and late onset Alzheimer disease: a post mortem study. Neuropathol. Appl. Neurobiol. 44, 298-313.

Tan, Y.L., Yuan, Y., and Tian, L. (2019). Microglial regional heterogeneity and its role in the brain. Mol. Psychiatry 2019252 25, 351-367.

Terry, R.D. (2000). Cell death or synaptic loss in Alzheimer disease. J. Neuropathol. Exp. Neurol. 59, 1118-1119.

Thion, M.S., Low, D., Silvin, A., Chen, J., Grisel, P., Schulte-Schrepping, J., Blecher, R., Ulas, T., Squarzoni, P., Hoeffel, G., et al. (2018). Microbiome Influences Prenatal and Adult Microglia in a Sex-Specific Manner. Cell 172, 500-516.e16.

Thompson, P.M., Hayashi, K.M., De Zubicaray, G.I., Janke, A.L., Rose, S.E., Semple, J., Hong, M.S., Herman, D.H., Gravano, D., Doddrell, D.M., et al. (2004). Mapping hippocampal and ventricular change in Alzheimer disease. Neuroimage 22, 1754-1766.

Trapnell, C., Cacchiarelli, D., Grimsby, J., Pokharel, P., Li, S., Morse, M., Lennon, N.J., Livak, K.J., Mikkelsen, T.S., and Rinn, J.L. (2014). The dynamics and regulators of cell fate 
decisions are revealed by pseudotemporal ordering of single cells. Nat. Biotechnol. 2014324 $32,381-386$.

Tremblay, M.-È., Stevens, B., Sierra, A., Wake, H., Bessis, A., and Nimmerjahn, A. (2011). The role of microglia in the healthy brain. J. Neurosci. 31, 16064-16069.

Turner, R.S. (2001). Alzheimer's disease in man and transgenic mice: Females at higher risk. Am. J. Pathol. 158, 797-801.

Valeeva, G., Tressard, T., Mukhtarov, M., Baude, A., and Khazipov, R. (2016). An Optogenetic Approach for Investigation of Excitatory and Inhibitory Network GABA Actions in Mice Expressing Channelrhodopsin-2 in GABAergic Neurons. J. Neurosci. 36, 5961.

Vegeto, E., Belcredito, S., Ghisletti, S., Meda, C., Etteri, S., and Maggi, A. (2006). The endogenous estrogen status regulates microglia reactivity in animal models of neuroinflammation. Endocrinology 147, 2263-2272.

Venturino, A., Schulz, R., De Jesús-Cortés, H., Maes, M.E., Nagy, B., Reilly-Andújar, F., Colombo, G., Cubero, R.J.A., Schoot Uiterkamp, F.E., Bear, M.F., et al. (2021). Microglia enable mature perineuronal nets disassembly upon anesthetic ketamine exposure or $60-\mathrm{Hz}$ light entrainment in the healthy brain. Cell Rep. 36.

Villa, A., Rizzi, N., Vegeto, E., Ciana, P., and Maggi, A. (2015). Estrogen accelerates the resolution of inflammation in macrophagic cells. Sci. Rep. 5.

Villa, A., Vegeto, E., Poletti, A., and Maggi, A. (2016). Estrogens, Neuroinflammation, and Neurodegeneration. Endocr. Rev. 37, 372.

Villa, A., Gelosa, P., Castiglioni, L., Cimino, M., Rizzi, N., Pepe, G., Lolli, F., Marcello, E., Sironi, L., Vegeto, E., et al. (2018). Sex-Specific Features of Microglia from Adult Mice. Cell Rep. 23, 3501-3511.

Wang, X., Zhang, C., Szábo, G., and Sun, Q.Q. (2013). Distribution of CaMKII $\alpha$ expression in the brain in vivo, studied by CaMKII $\alpha$-GFP mice. Brain Res. 1518, 9-25.

Wiesman, A.I., Mundorf, V.M., Casagrande, C.C., Wolfson, S.L., Johnson, C.M., May, P.E., Murman, D.L., and Wilson, T.W. (2021). Somatosensory dysfunction is masked by variable cognitive deficits across patients on the Alzheimer's disease spectrum. EBioMedicine 73, 
103638.

Wong, T., Zhang, X.L., Nassiri Asl, M., Wu, C.P., Carlen, P.L., and Zhang, L. (2005).

Postnatal development of intrinsic GABAergic rhythms in mouse hippocampus.

Neuroscience 134, 107-120.

Wu, Y., Dissing-Olesen, L., MacVicar, B.A., and Stevens, B. (2015). Microglia: Dynamic Mediators of Synapse Development and Plasticity. Trends Immunol. 36, 605-613.

Yang, J.M., Zhang, J., Yu, Y.Q., Duan, S., and Li, X.M. (2014). Postnatal development of 2 microcircuits involving fast-spiking interneurons in the mouse prefrontal cortex. Cereb. Cortex 24, 98-109.

Yanguas-Casás, N., Crespo-Castrillo, A., de Ceballos, M.L., Chowen, J.A., Azcoitia, I., Arevalo, M.A., and Garcia-Segura, L.M. (2018). Sex differences in the phagocytic and migratory activity of microglia and their impairment by palmitic acid. Glia $66,522-537$.

Young, K., and Morrison, H. (2018). Quantifying microglia morphology from photomicrographs of immunohistochemistry prepared tissue using imagej. J. Vis. Exp. 2018, 57648.

Zarow, C., Lyness, S.A., Mortimer, J.A., and Chui, H.C. (2003). Neuronal Loss Is Greater in the Locus Coeruleus Than Nucleus Basalis and Substantia Nigra in Alzheimer and Parkinson Diseases. Arch. Neurol. 60, 337-341.

Zusso, M., Methot, L., Lo, R., Greenhalgh, A.D., David, S., and Stifani, S. (2012).

Regulation of postnatal forebrain amoeboid microglial cell proliferation and development by the transcription factor runx1. J. Neurosci. 32, 11285-11298. 
bioRxiv preprint doi: https://doi.org/10.1101/2021.11.30.470\&9:this kersiop posted December 1, 2021. The copyright holder for this

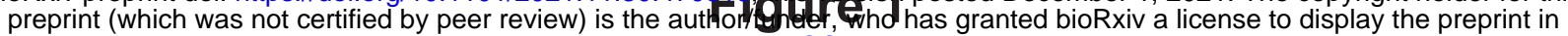
A Microglial heterogeneity across brain r
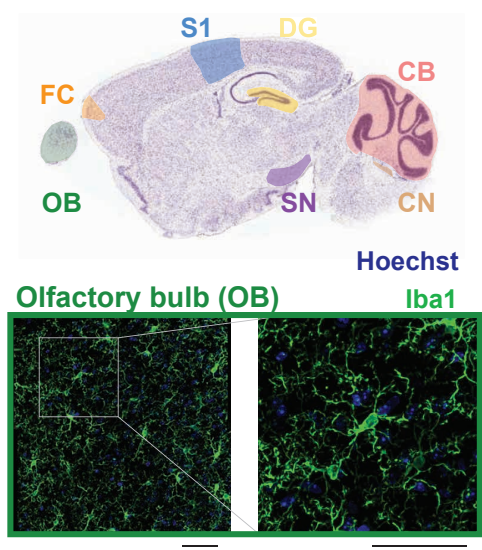

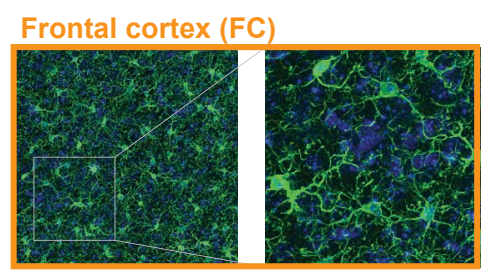

Dentate gyrus (DG)
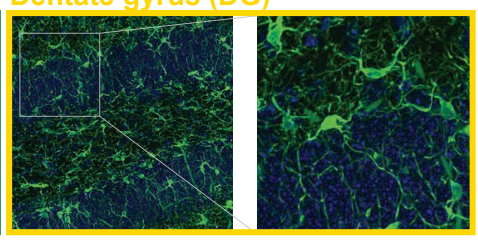

Somatosensory cortex (S1)

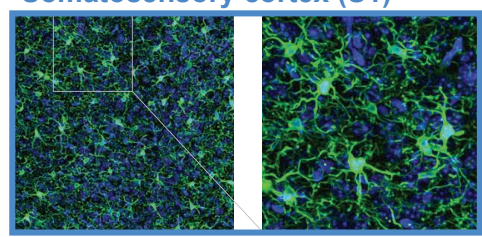

Substantia nigra (SN)

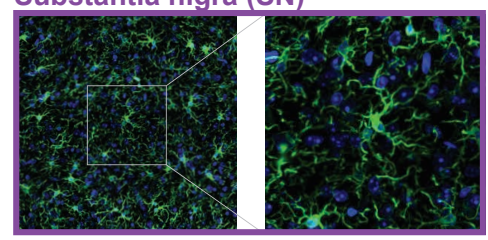

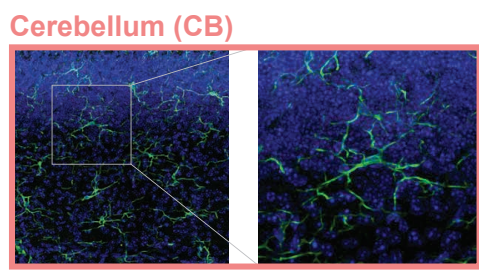

Cochlear nucleus $(\mathrm{CN})$

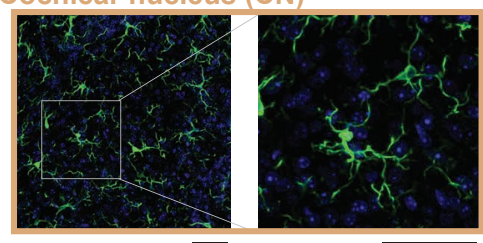

B MorphOMICs-part 1 - Topological morphological descriptor

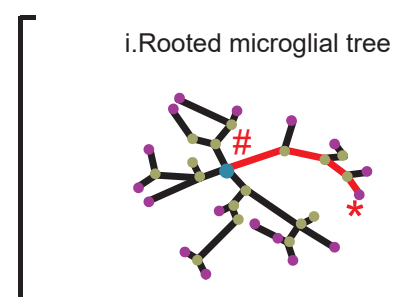

$\begin{aligned} \text { Root }=\text { Soma } & \text { Leaf }=\text { terminal point } \\ \text { Node }=\text { branching point } & \text { Edge }=\text { process }\end{aligned}$
iii.Persistence diagram iv.Persistence image $]$

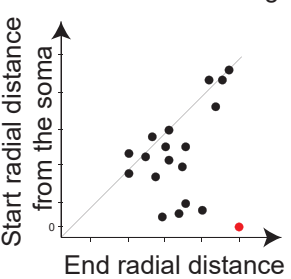

from the soma

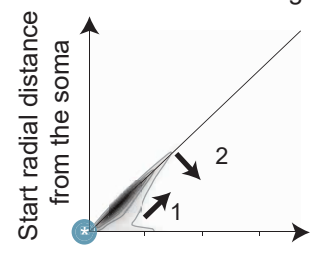

End radial distance from the soma

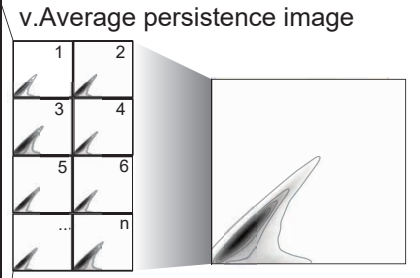

$J_{n}$

C TMD: Average persistence images of microglia across brain regions

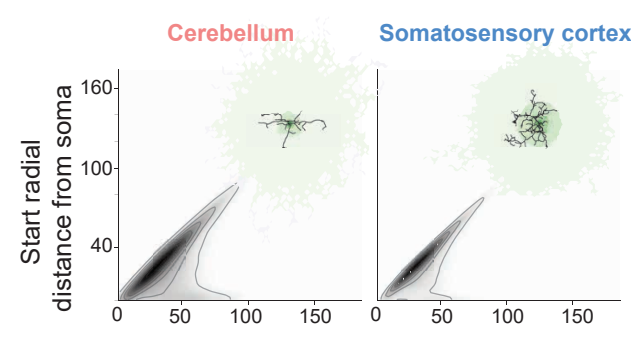

D MorphOMICs part 2- Bootstrapping

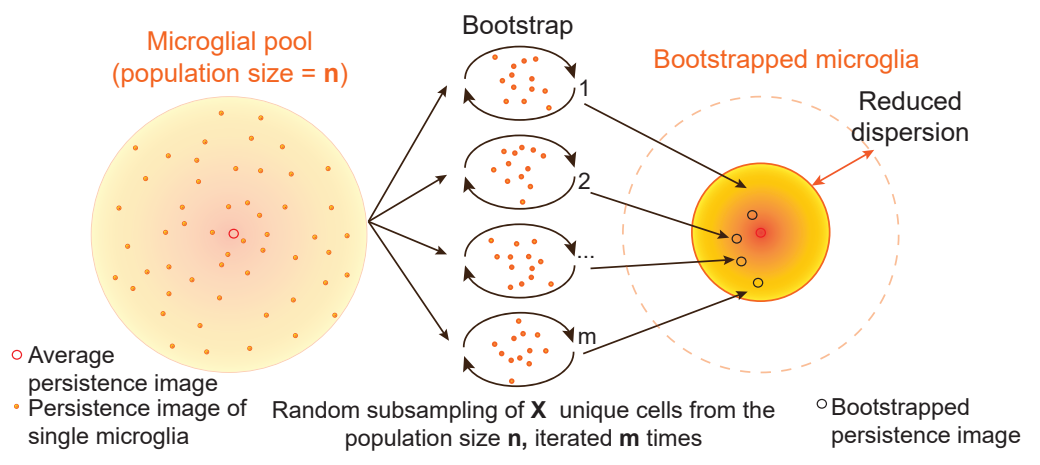

E MorphoOMICs part 3-Dimension reduction and visualization

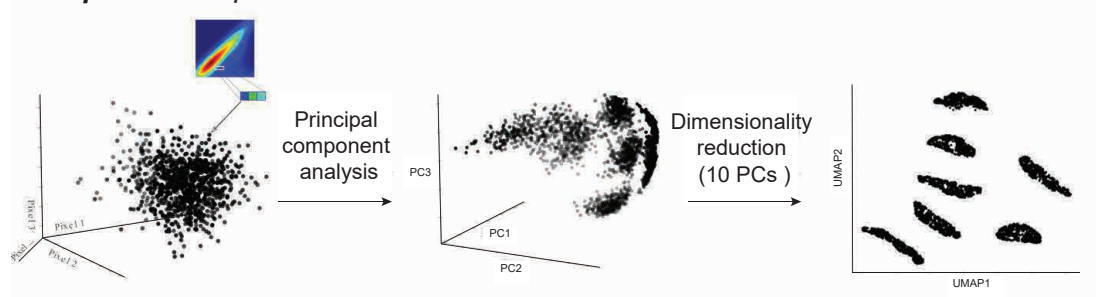

F Spatial heterogeneity of adult microglia

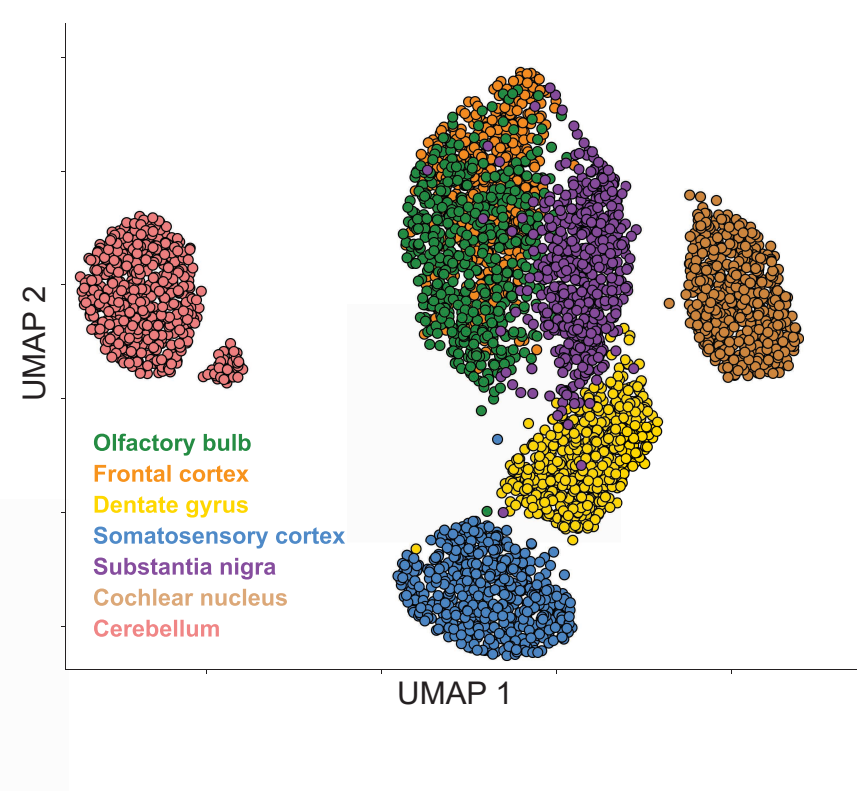


bioRxiv preprint doi: https://doi.org/10.1101/2021.11.34-40610: thistyersion posted December 1, 2021. The copyright holder for this

preprint (which was not certified by peer review) is the lghenffudar, who has granted bioRxiv a license to display the preprint in perpetuity. It is made available under aCC-BY-ND 4.0 International license.

A
OB
Frontal cortex (FC)
Dentate gyrus (DG)
Cerebellum (CB)
Olfactory bulb (OB) Substantia nigra (SN)
Cochlear nucleus (CN)

B Sexual dimorphism of microglial morphology

C Spatial heterogeneity of ovarectomized females

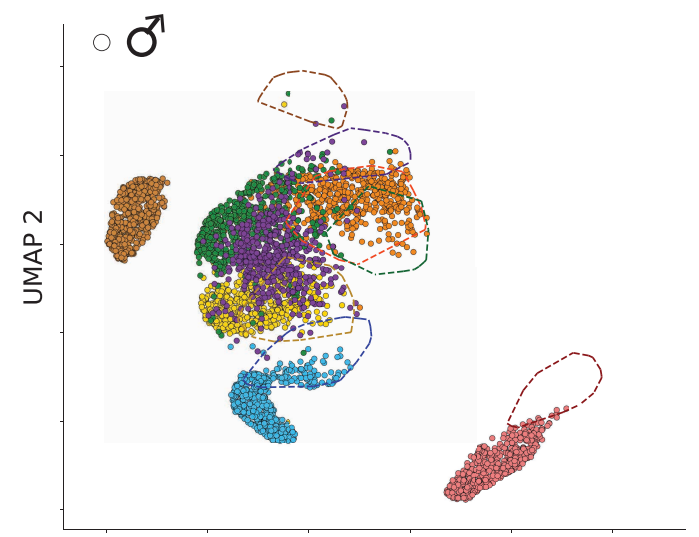

UMAP 1

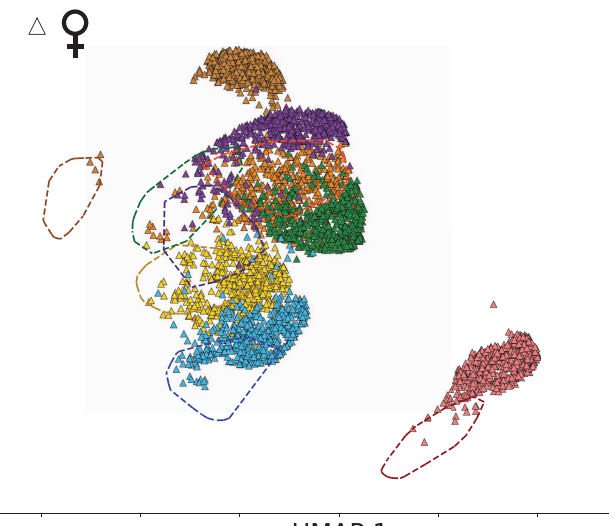

UMAP 1

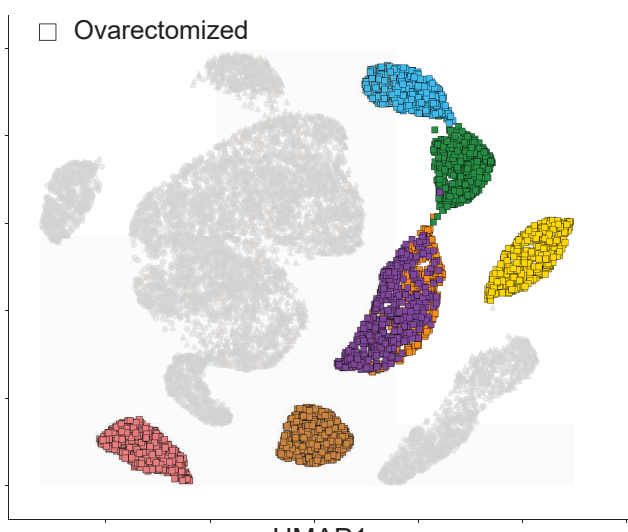

UMAP1 
bioRxiv preprint doi: https://doi.org/10.1101/2021.11.30.47/8jatulfrersi8n posted December 1, 2021. The copyright holder for this

preprint (which was not certified by peer review) is the authoifunder, who has granted bioRxiv a license to display the preprint in

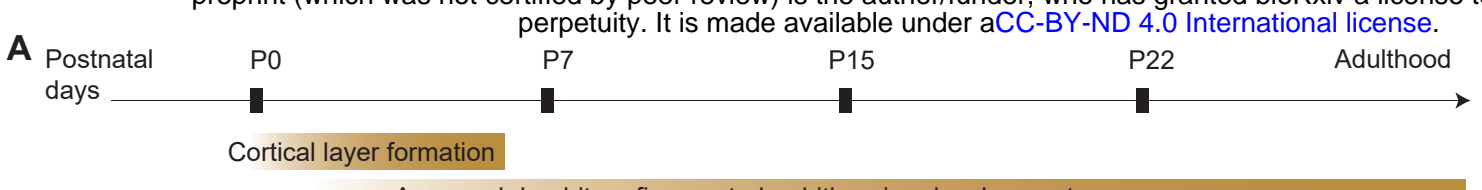

Axon and dendrite refinement, dendritic spine development

Synapse setting, maturation and pruning

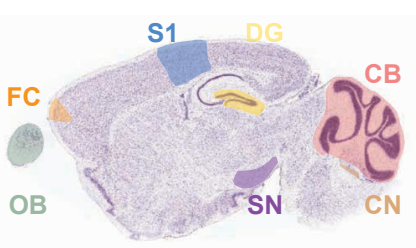

B Morphological plasticity during the postnatal development

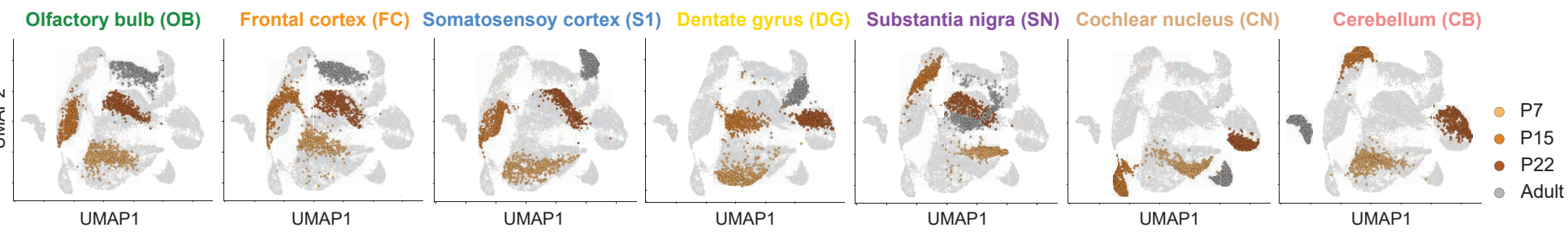

C Morphological heterogeneity of microglia - development

P7

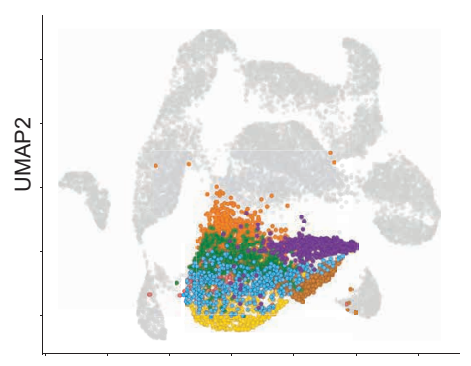

UMAP1
P15

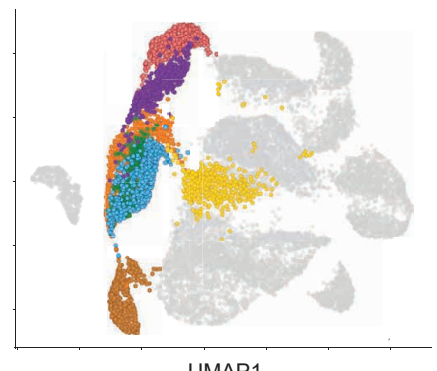

UMAP1
P22
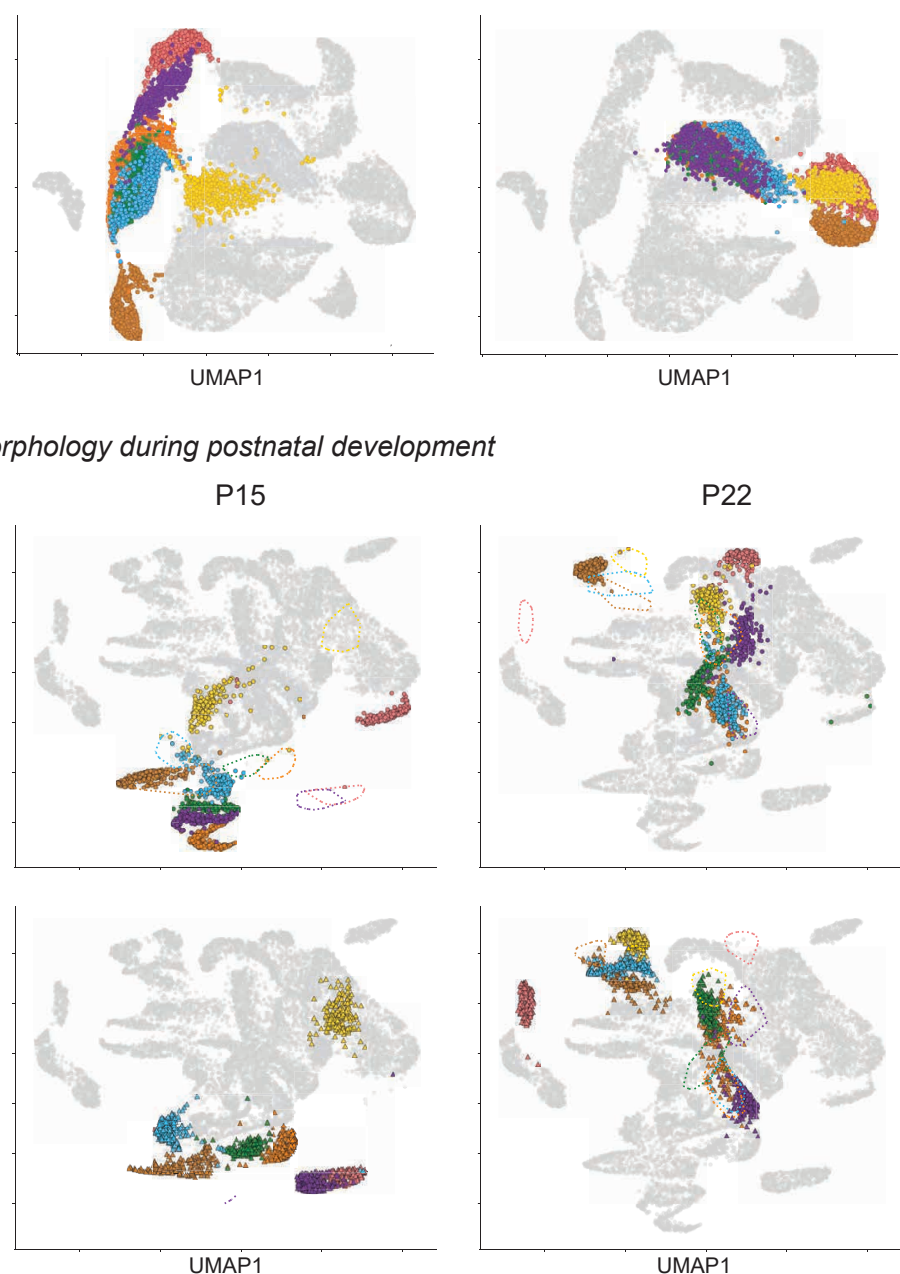

$\mathrm{P} 22$
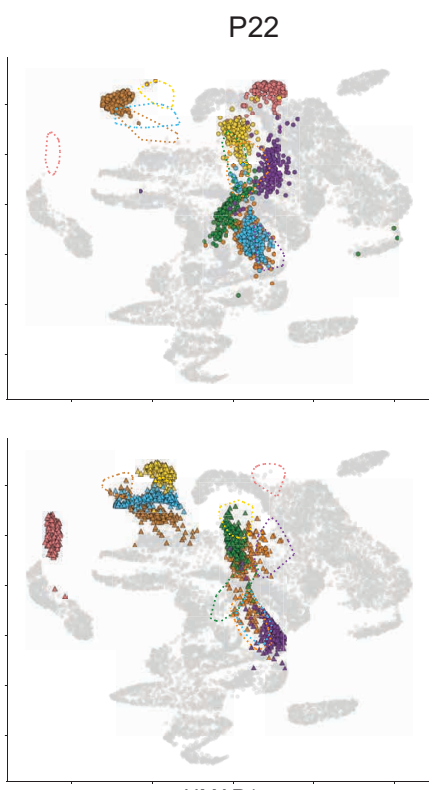

UMAP1
UMAP1
Adult

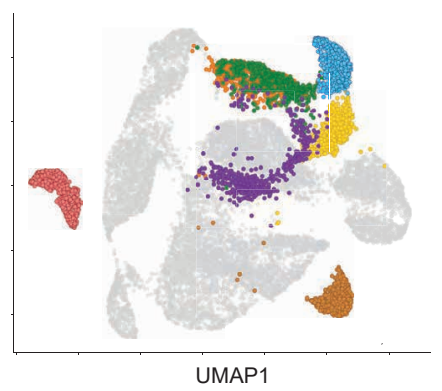

D Sexual dimorphism of microglial morphology during postnatal development
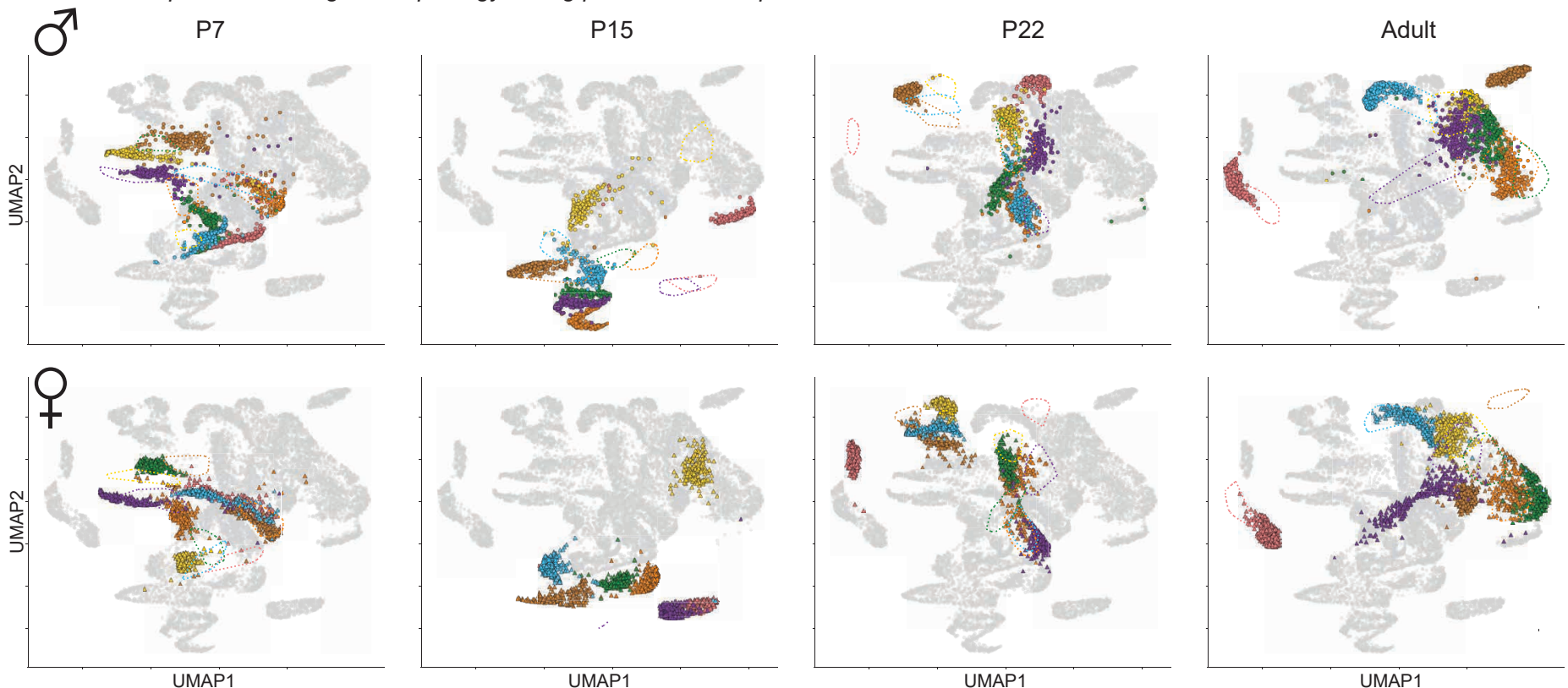

E Palantir region specific morphological trajectory of microglia during postnatal development

Olfactory bulb (OB) Frontal cortex (FC) Somatosensoy cortex (S1) Dentate gyrus (DG) Substantia nigra (SN) Cochlear nucleus (CN) Cerebellum (CB)
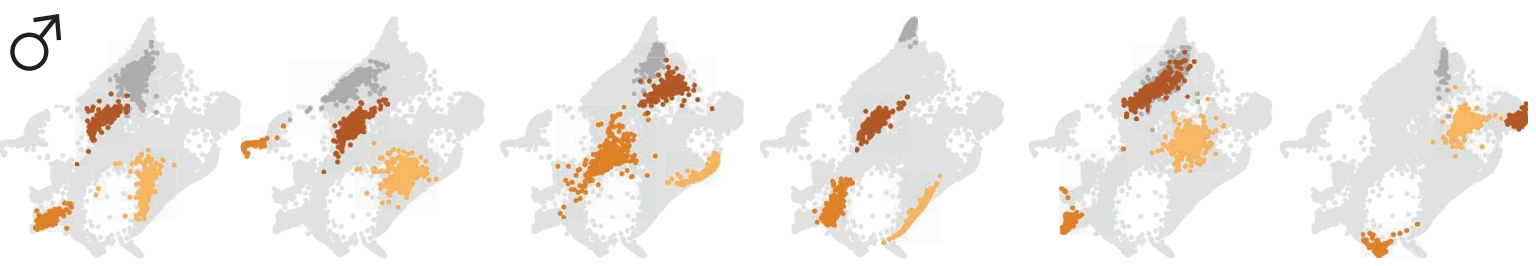

:
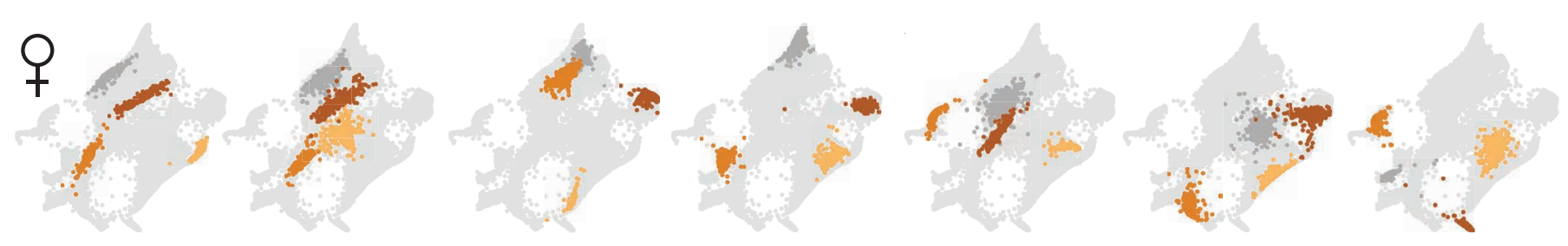
bioRxiv preprint doi: https://doi.org/10.1101/2021.11.30.47/6igtulshersqn posted December 1, 2021. The copyright holder for this

preprint (which was not certified by peer review) is the authorffunder, who has granted bioRxiv a license to display the preprint in

A perpetuity. It is made available under aCC-BY-ND 4.0 International license.

$5 x F A D$

3 months

6 months

S1

OB

SN

$A \beta_{42}$ accumulation Intraneuronal

B

Morphological heterogeneity in 5xFAD

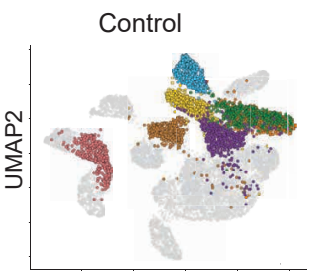

UMAP1

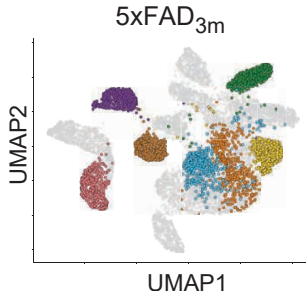

UMAP1

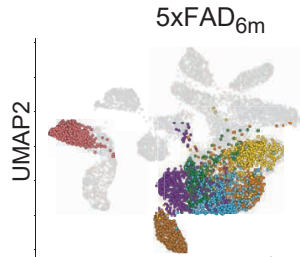

UMAP1

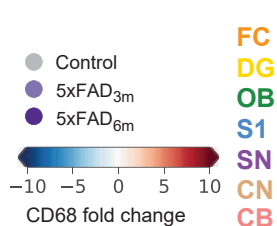

q
Merged

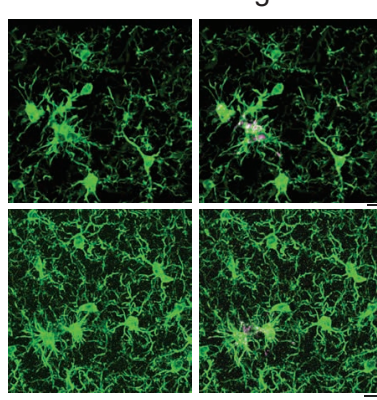

Palantir

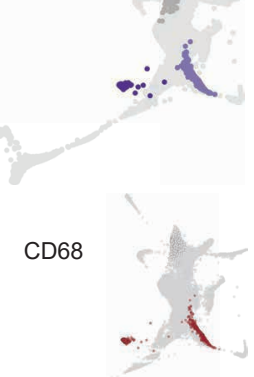

C
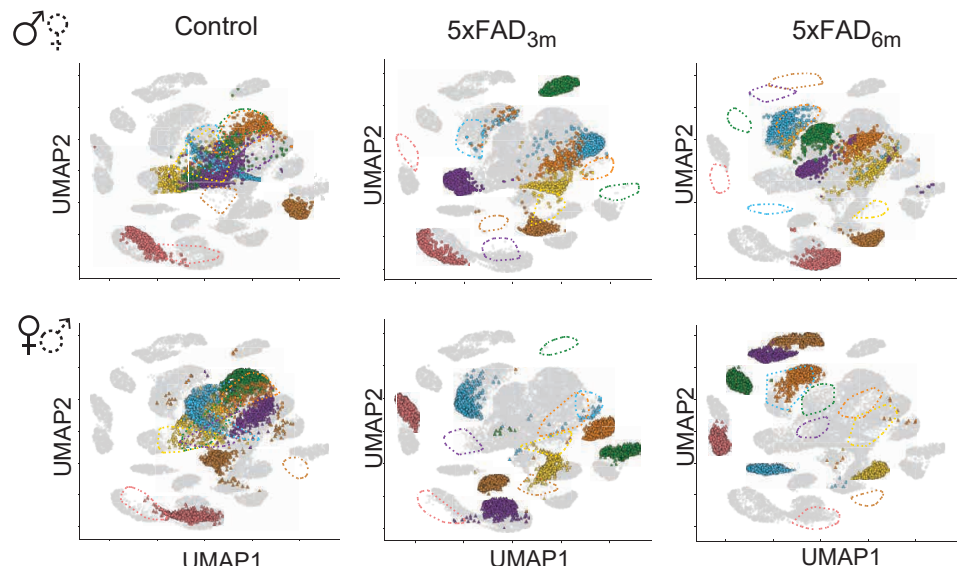

$\sigma^{\top}$

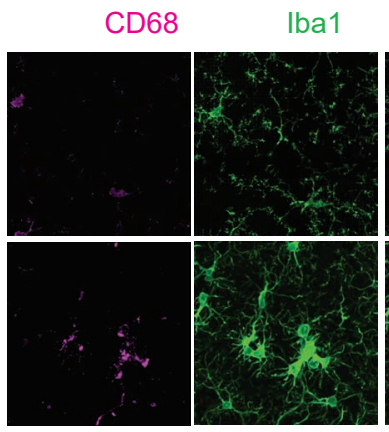

Merged
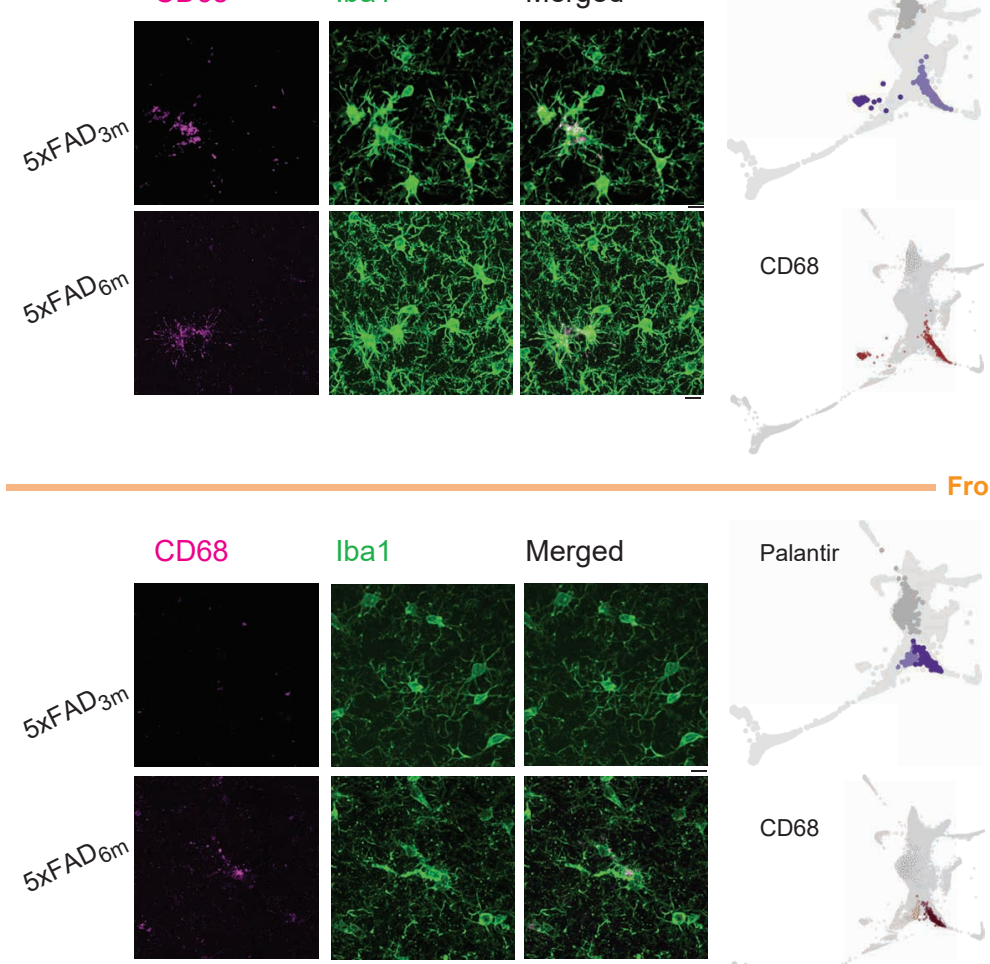

Palantir

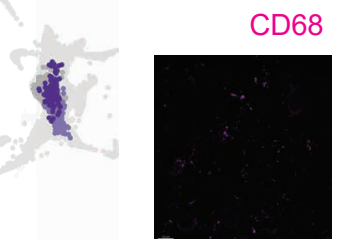

CD68
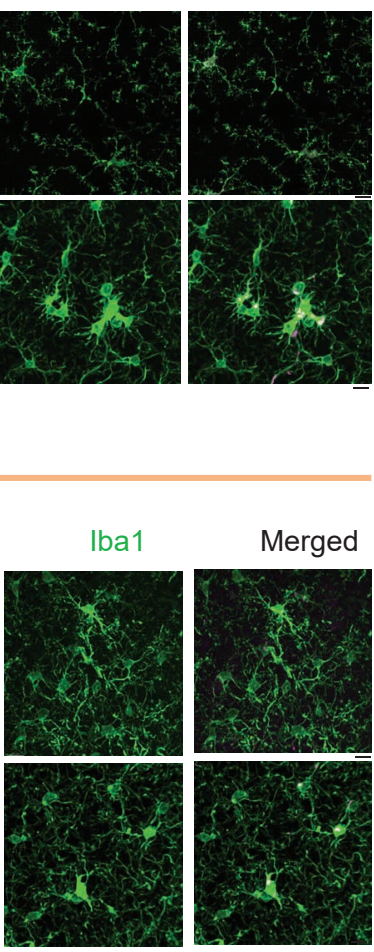

CD68

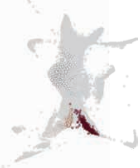

CD68
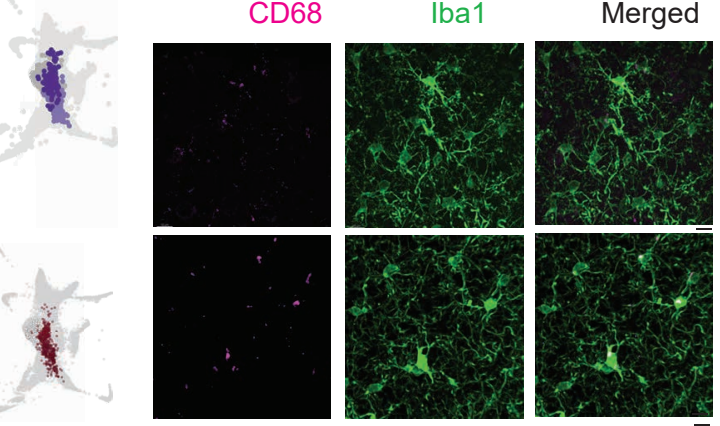

CD68

Iba1

Merged
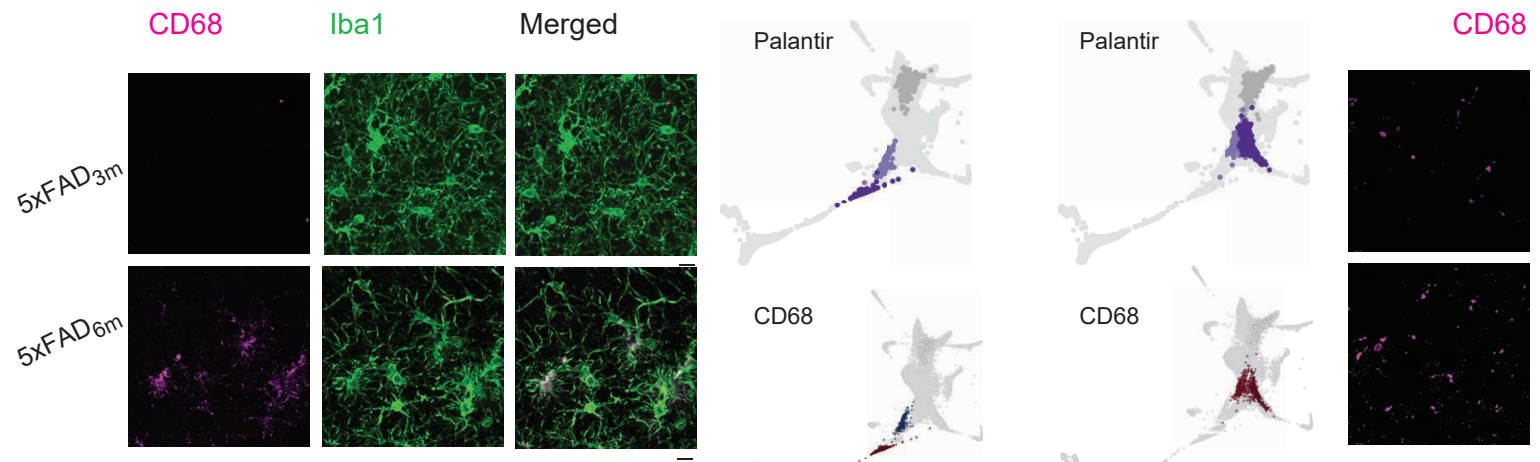

Iba1

Merged
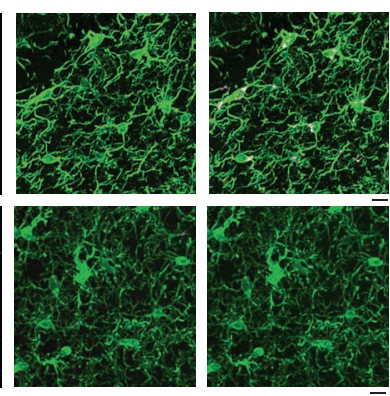
bioRxiv preprint doi: https://doi.org/10.1101/2021.11.30.47Fig bldersEn posted December 1, 2021. The copyright holder for this

preprint (which was noteertifiediby peer dreview) is the authorttunder, who has granted bioRxiv a license to display the preprint in

A
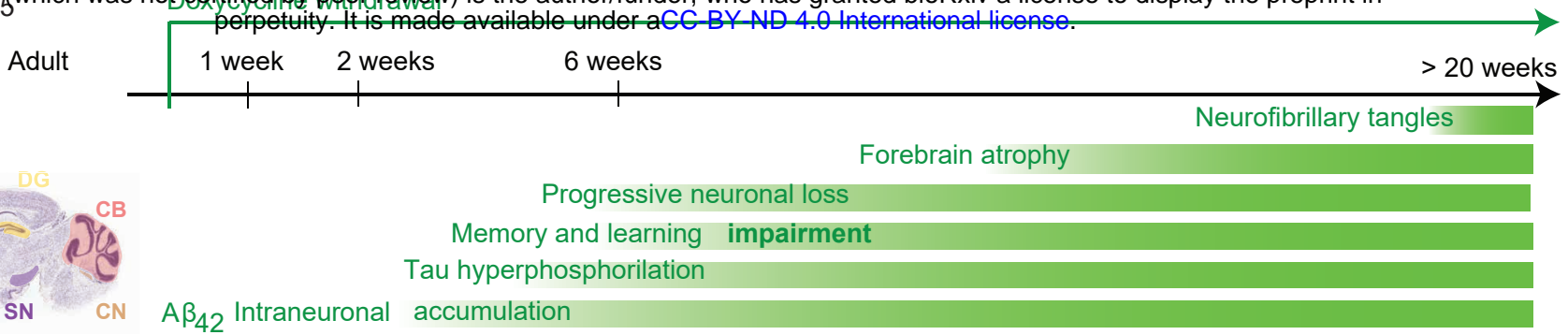

B

Morphological heterogeneity in CK-p25

C Sexual dimorphism of microglial morphology in CK-p25
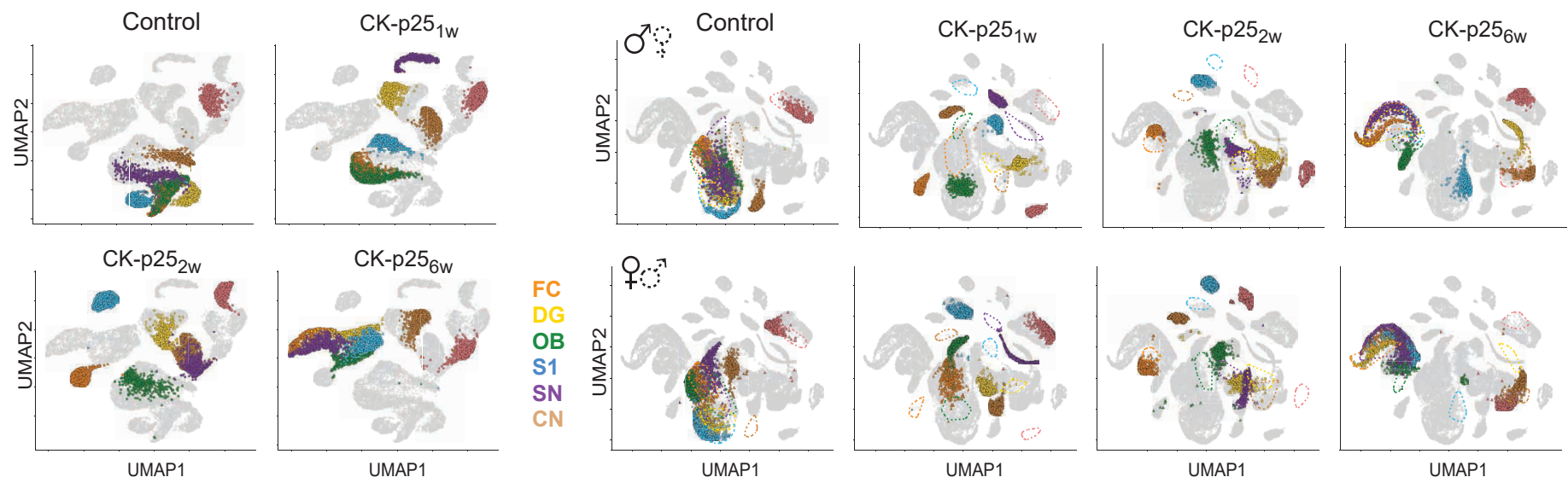

D Morphological trajectory and CD68 expression CD68 lba1 Merged

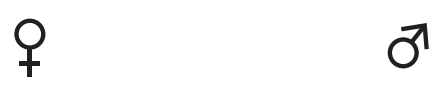

CD68

Iba1 Merged
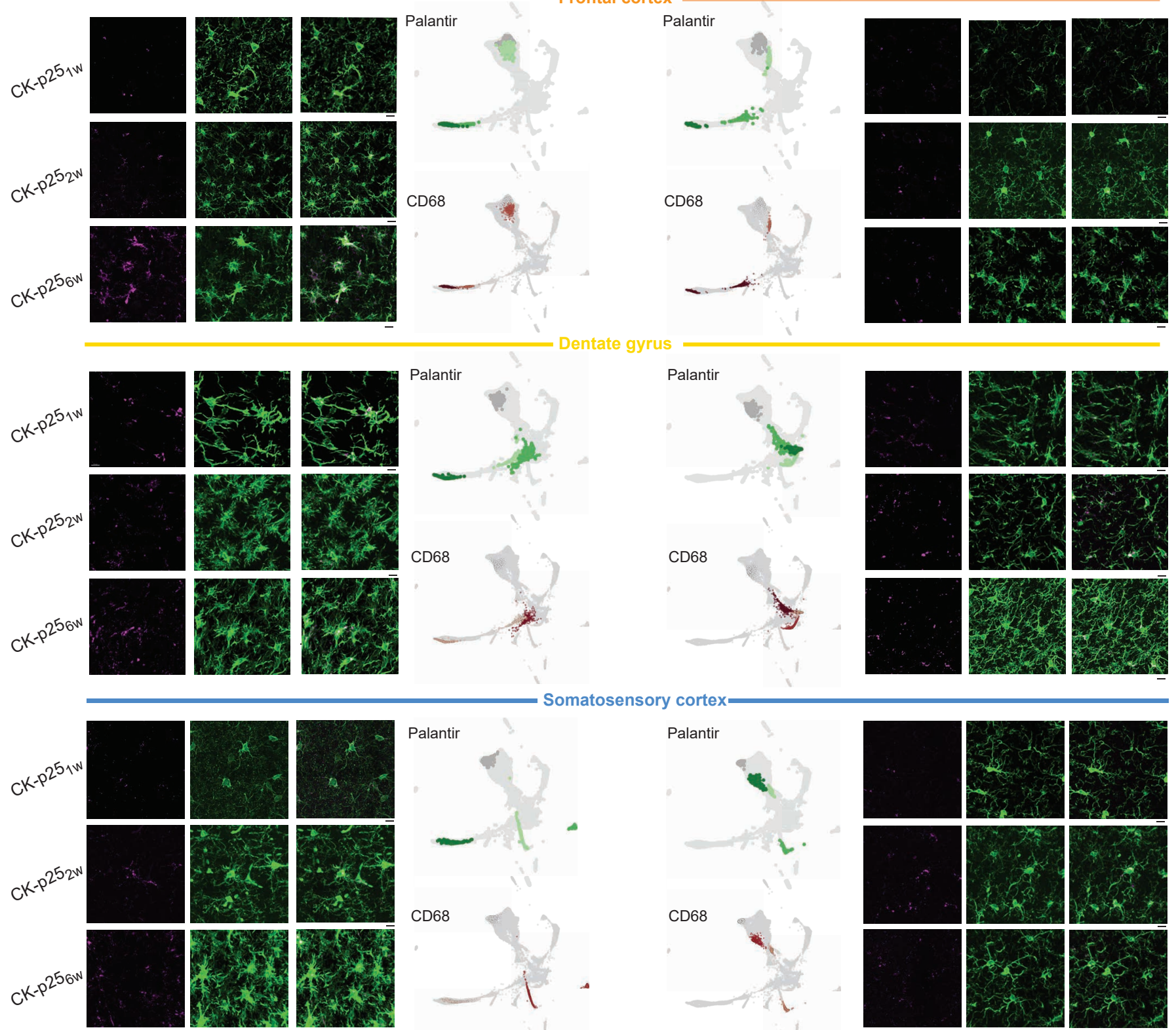
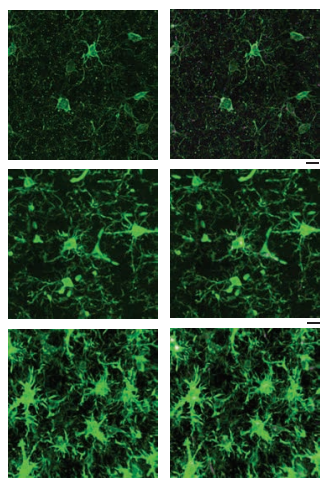

Palantir

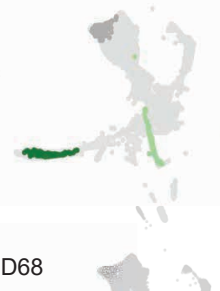

CD68

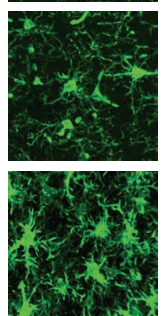

Somatosensory cortex
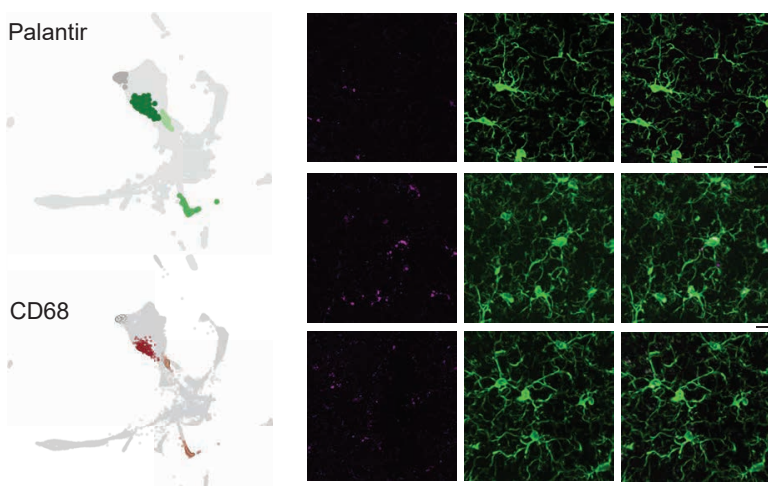

Control

- CK-p $25_{2 w}$

- CK-p256w

$\begin{array}{lllll}10 & -5 & 0 & 5 & 10\end{array}$

CD68 fold change 
bioRxiv preprint doi: https://doi.org/10.1101/2021.11.30.4Fäig thiper fon posted December 1, 2021. The copyright holder for this

preprint (which was not certified by peer review) is the authotfunder, who has granted bioRxiv a license to display the preprint in perpetuity. It is made available under aCC-BY-ND 4.0 International license.

A Comparison of females morphological trajectories in development and disease in the most affected regions

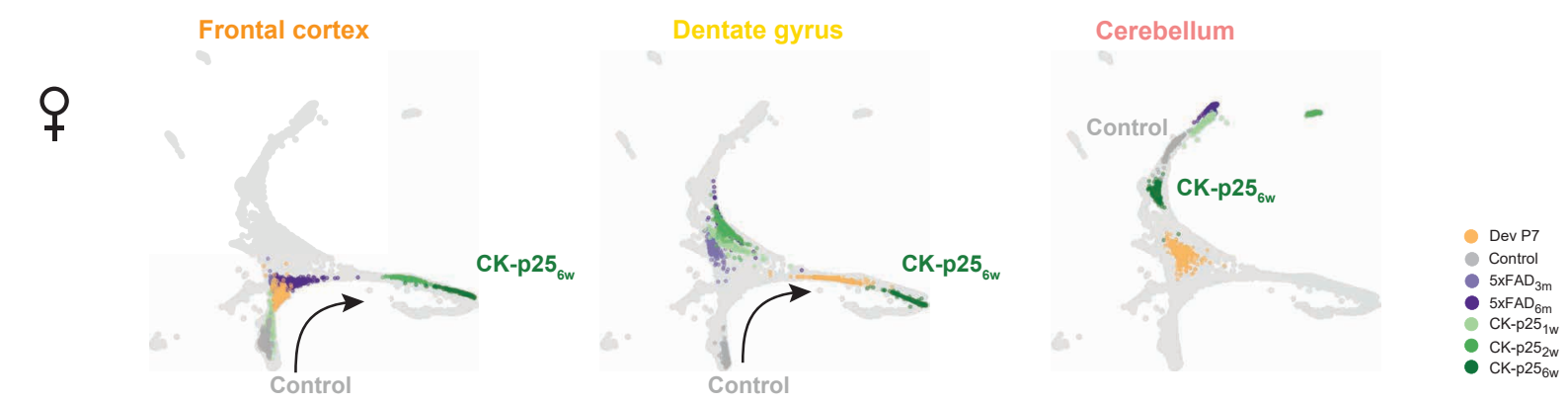

B Comparison of males morphological trajectories in development and disease in the most affected regions
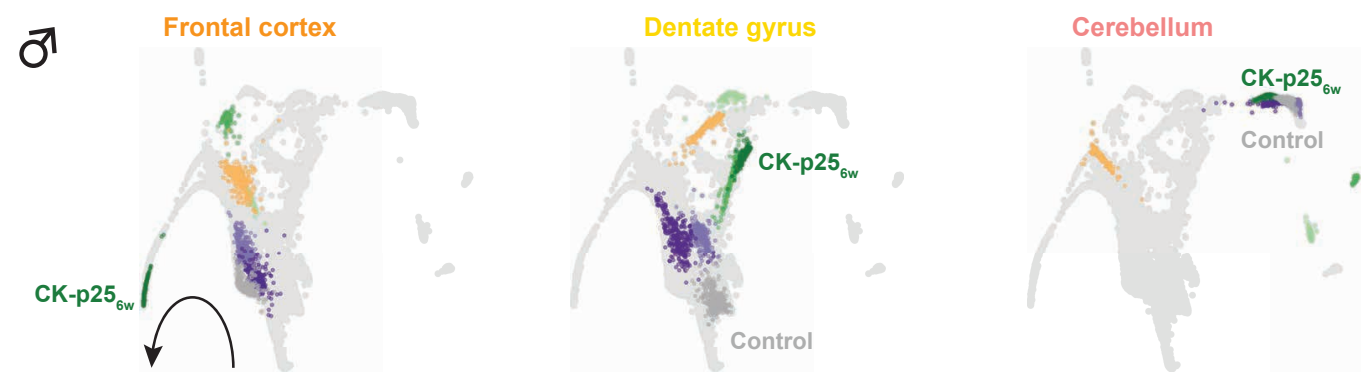


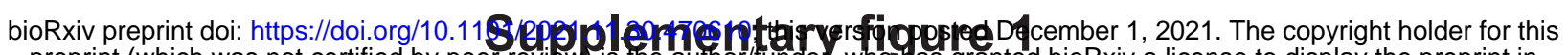

preprint (which was not certified by peer revieun) is the preprint in perpetuity. It is made available under aCC-BY-ND 4.0 International license.

A Common morphometric classifiers
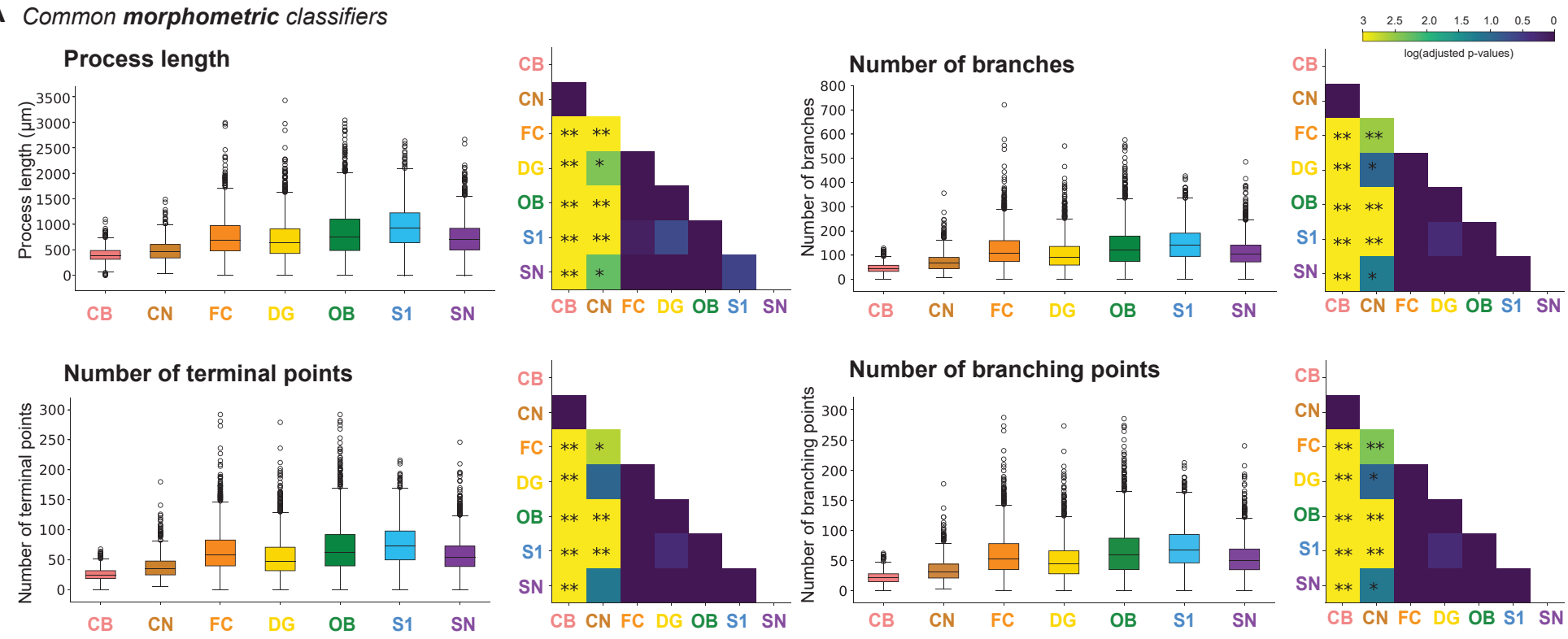

B TMD: Distance matrix

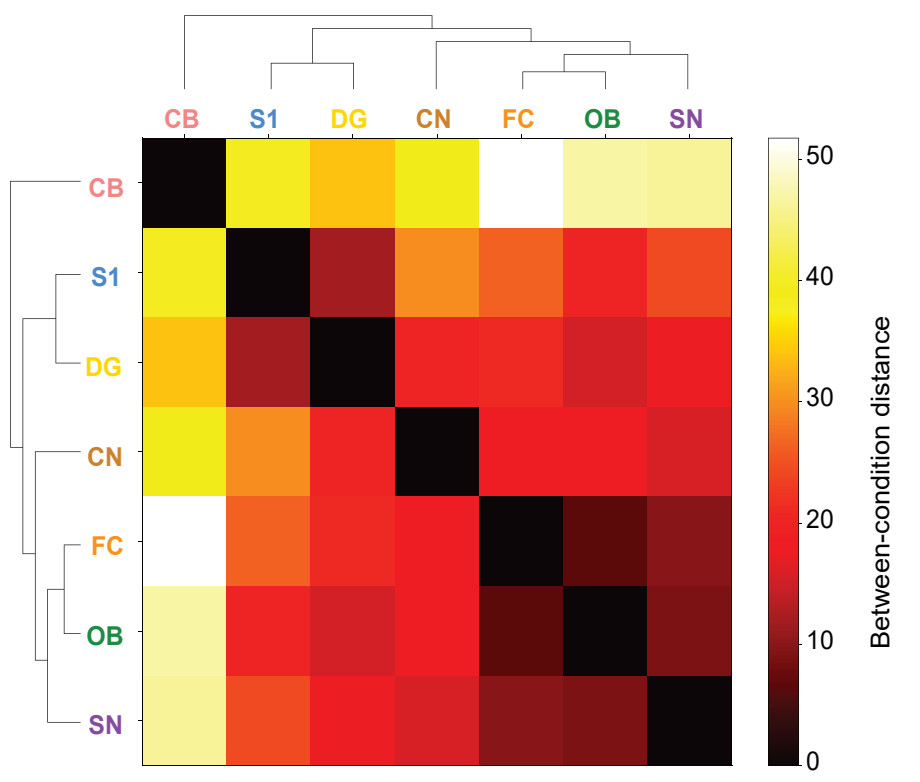

\section{TMD:}

Intrinsic spatial heterogeneity
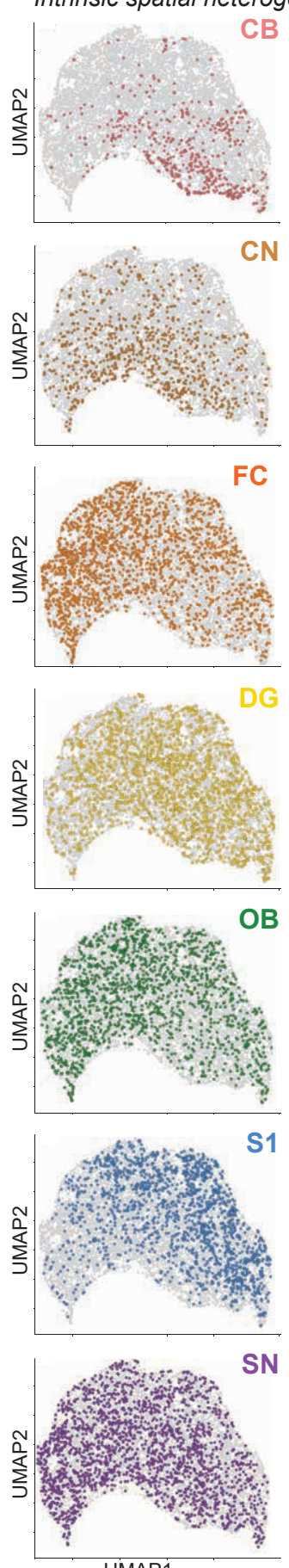

D TMD:

Distribution of the biological triplicate
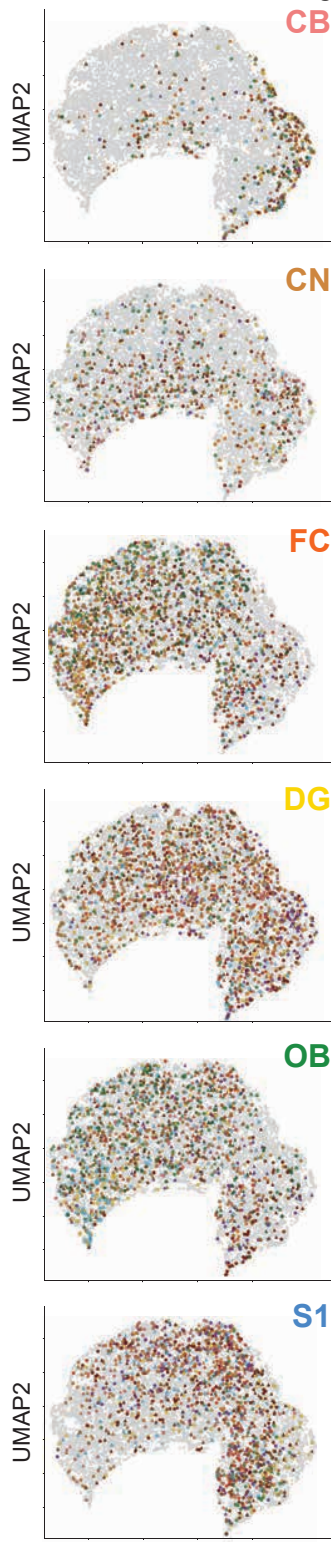

SN

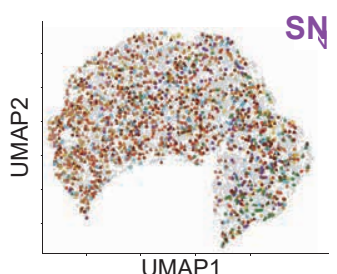




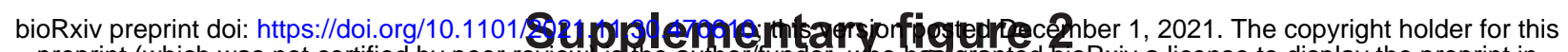

preprint (which was not certified by peer review) is the author/funder, who has granted bioRxiv a license to display the preprint in

A MorphOMICs :

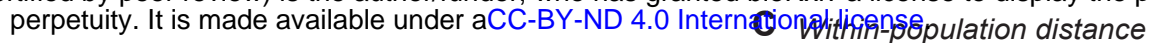

Bootstrap and variability reduction

Microglial bootstrap to population size ratio $(x / n)$

(Ratio between the number of microglia in the bootstrapped persistence images $\mathbf{x}$ and the starting population size $\mathbf{n}$ )

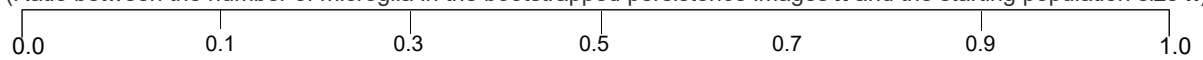

Microglial population 1 Microglial population 2
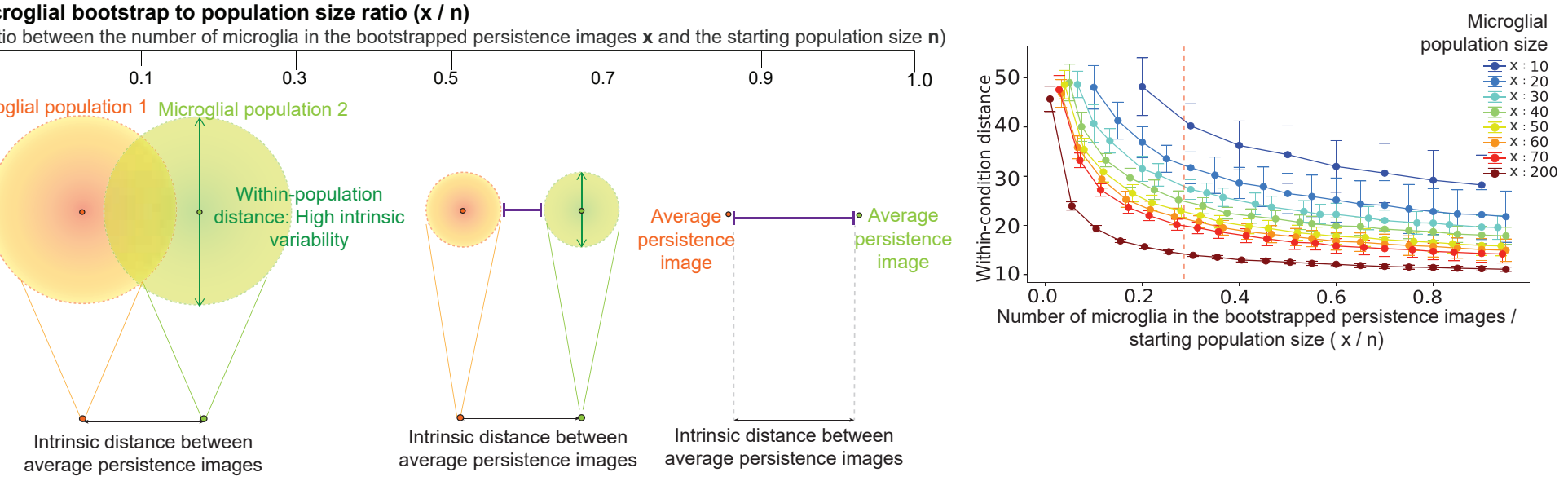

average persistence images

average persistence images average persistence images

B Data segregation for different bootstrap (x)-to-population size (n)
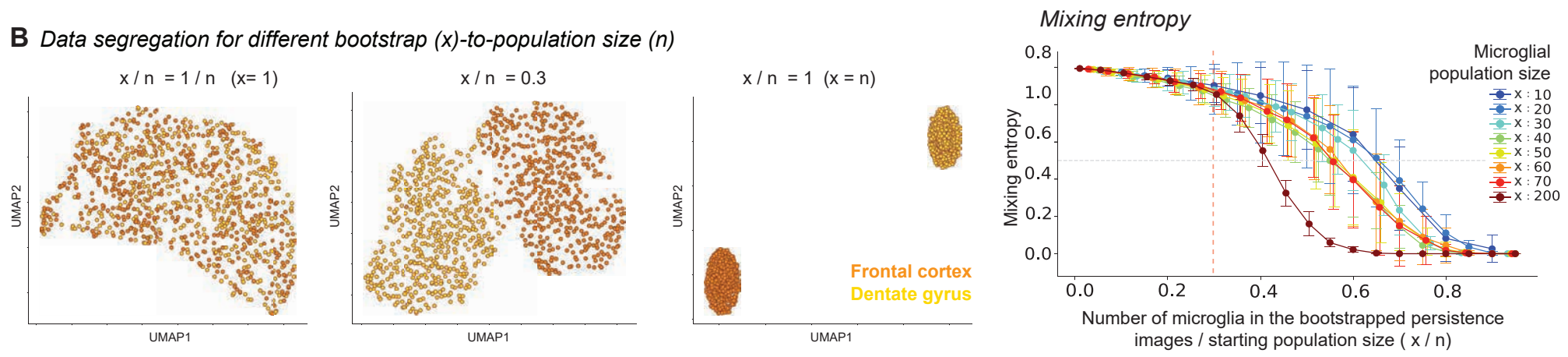

D Mixing entropy and within-condition distance

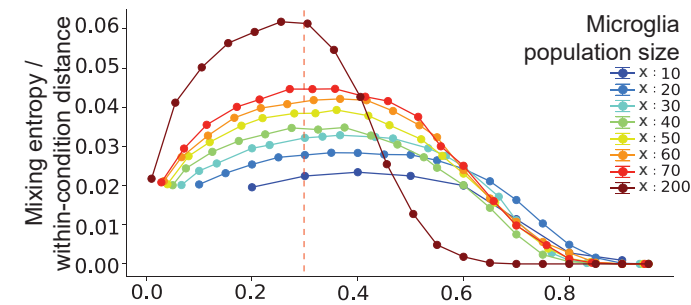

Number of microglia in the bootstrapped persistence images / starting population size $(x / n)$
E Female/male ratio
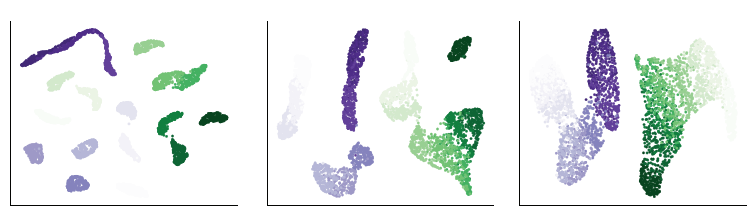

Microglia bootstrapping size / pool size

(Ratio between the number of microglia in the bootstrapped persistence images and the number of microglia in the pool)$$
1.0
$$

0.7

0.5

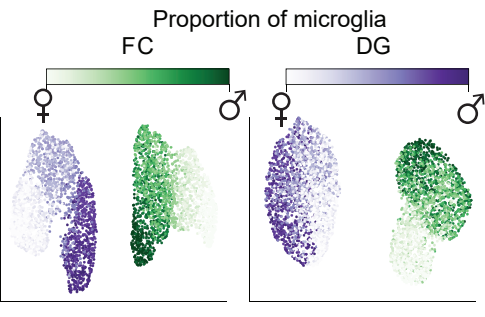
0.0

\section{F UMAP hyperparameters}

n_neighbors: 100 min_dist: 700

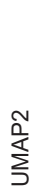

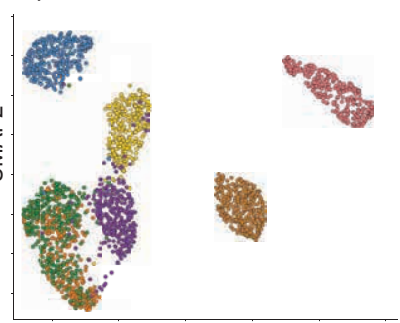

n_neighbors: 100

min dist: 700

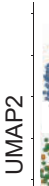

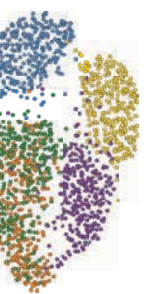

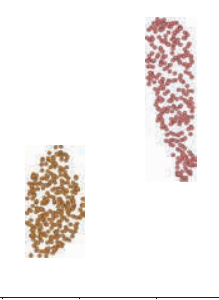

UMAP1

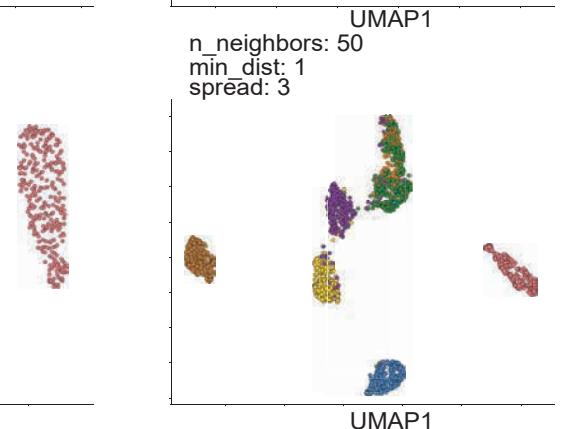

n_neighbors: 50

min_dist: 700

spread: 3

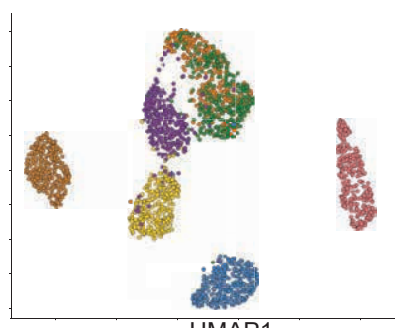

n_neighbors: 50

min dist:

UMAP1
G $t-S N E$

n_neighbors: 10

min_dist: 700

spread: 3

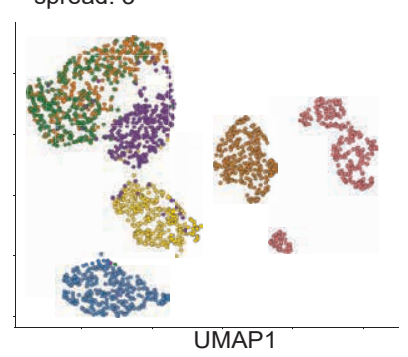

n_neighbors: 50

min_dist: 1

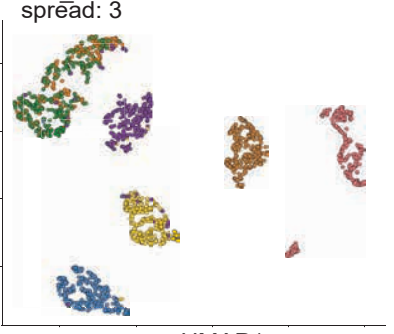

UMAP1

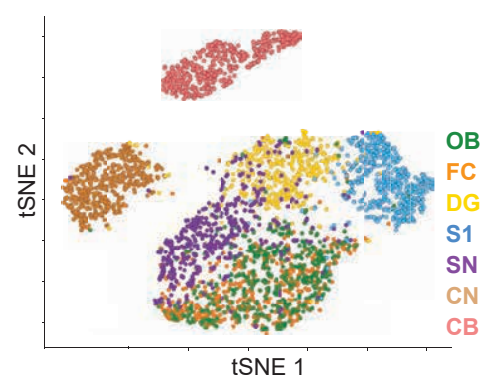

H Stable ranks

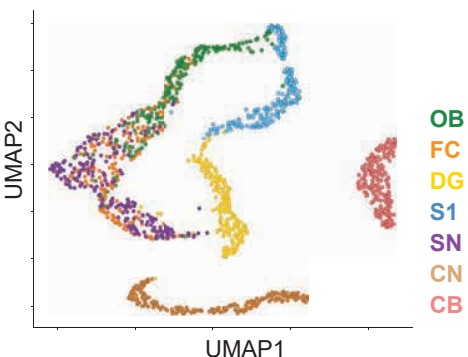


bioRxiv preprint doi: https://do erg/10.11p1/2021.11 30.470610 this version posted December 1, 2021. The copyright holder for this

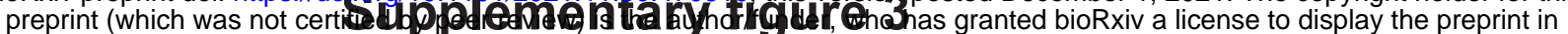
perpetưity. It is made available under aCC-BY-ND 4.0 International license.

A

Classification accuracy of microglial morphology with stable ranks

No bootstrapping

$\mathrm{CB}$.

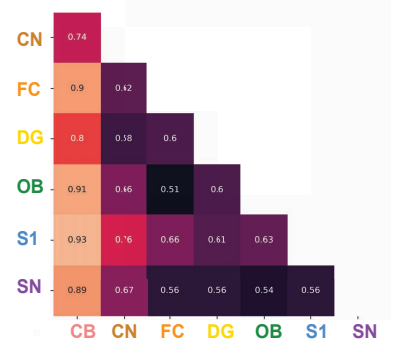

Bootstrapping with sample size 10

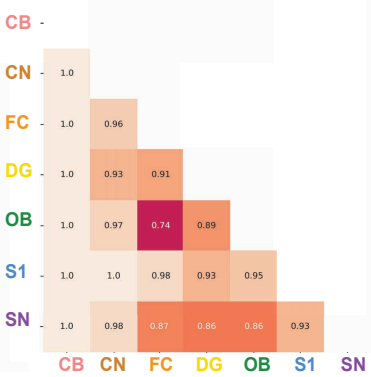

Bootstrapping with sample size 20

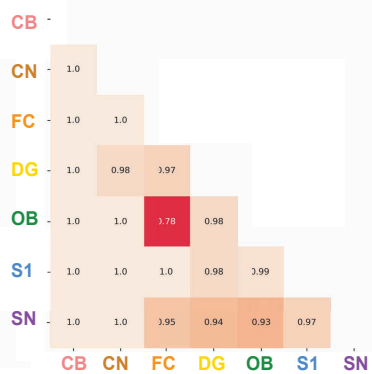

Bootstrapping with sample size 50

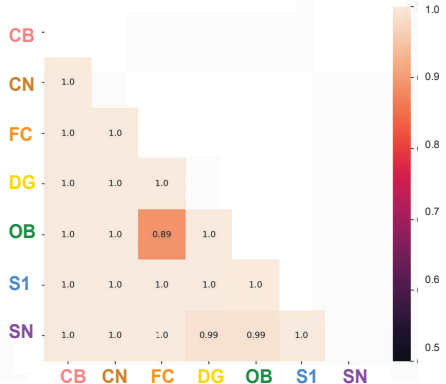

B

Sholl analysis to describe microglial morphology

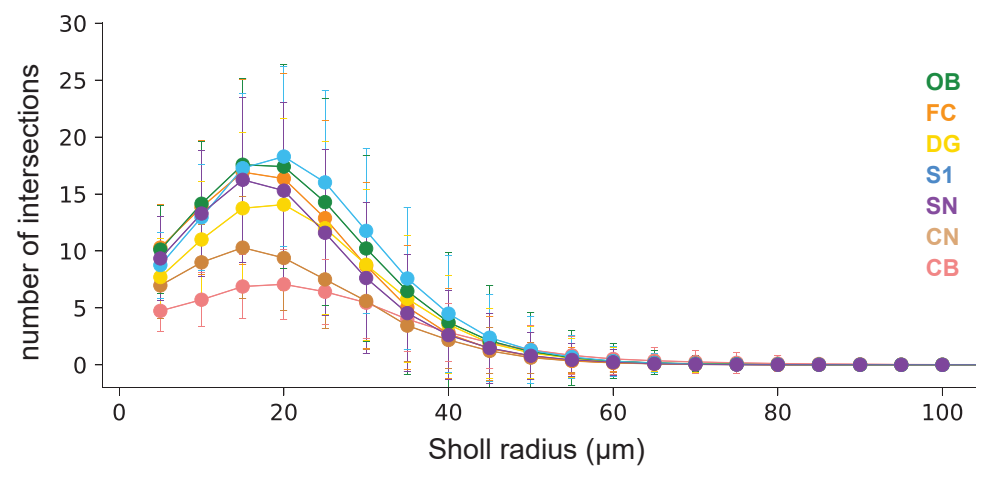

C

Bootstrap application on Sholl analysis
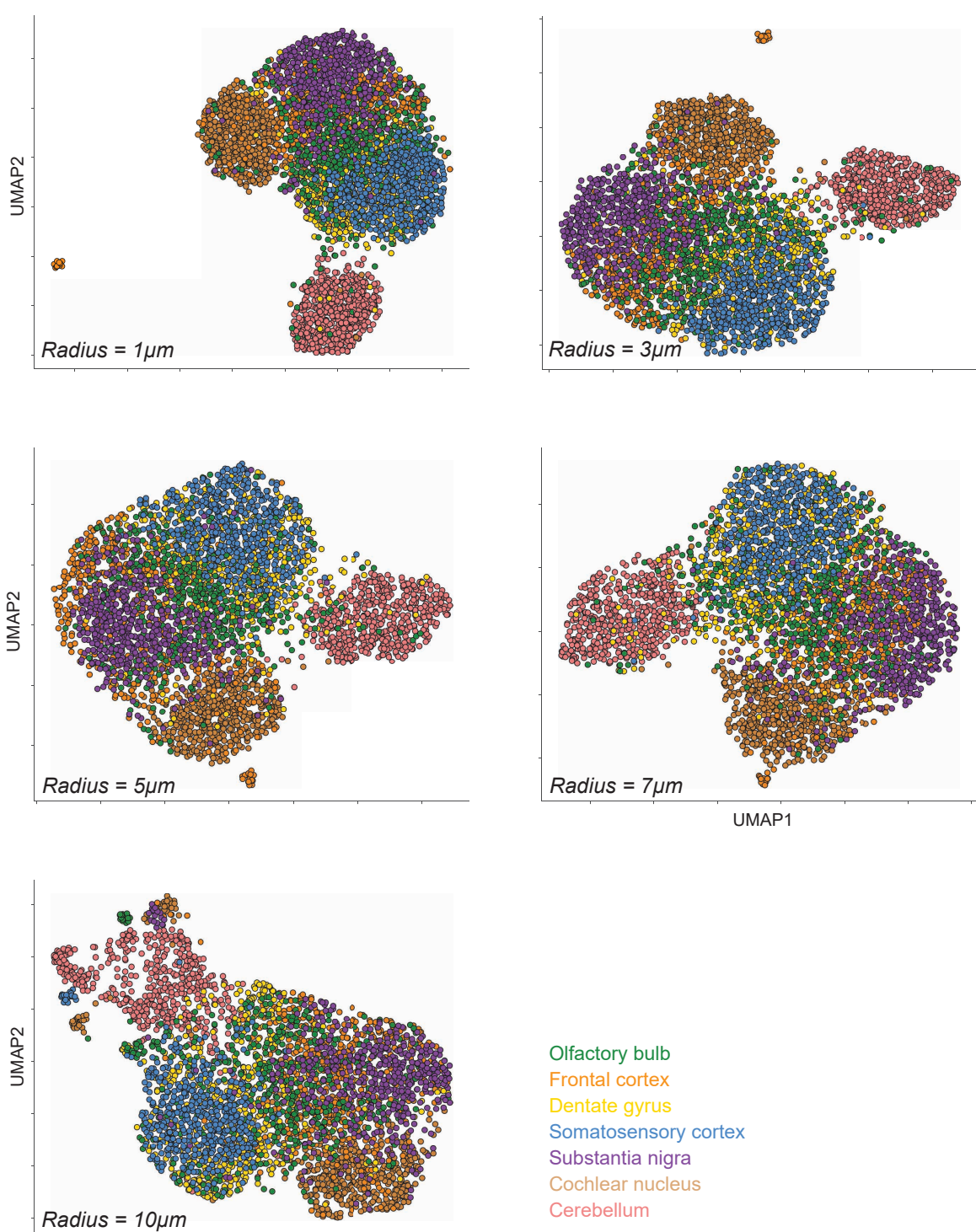

Olfactory bulb

Frontal cortex

Dentate gyrus

Somatosensory cortex

Substantia nigra

Cochlear nucleus

Cerebellum 
A

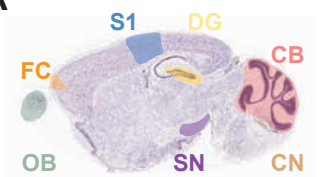

C Sexually dimorphic morphological plasticity during the postnatal development

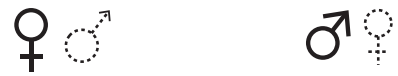

Frontal cortex (FC)
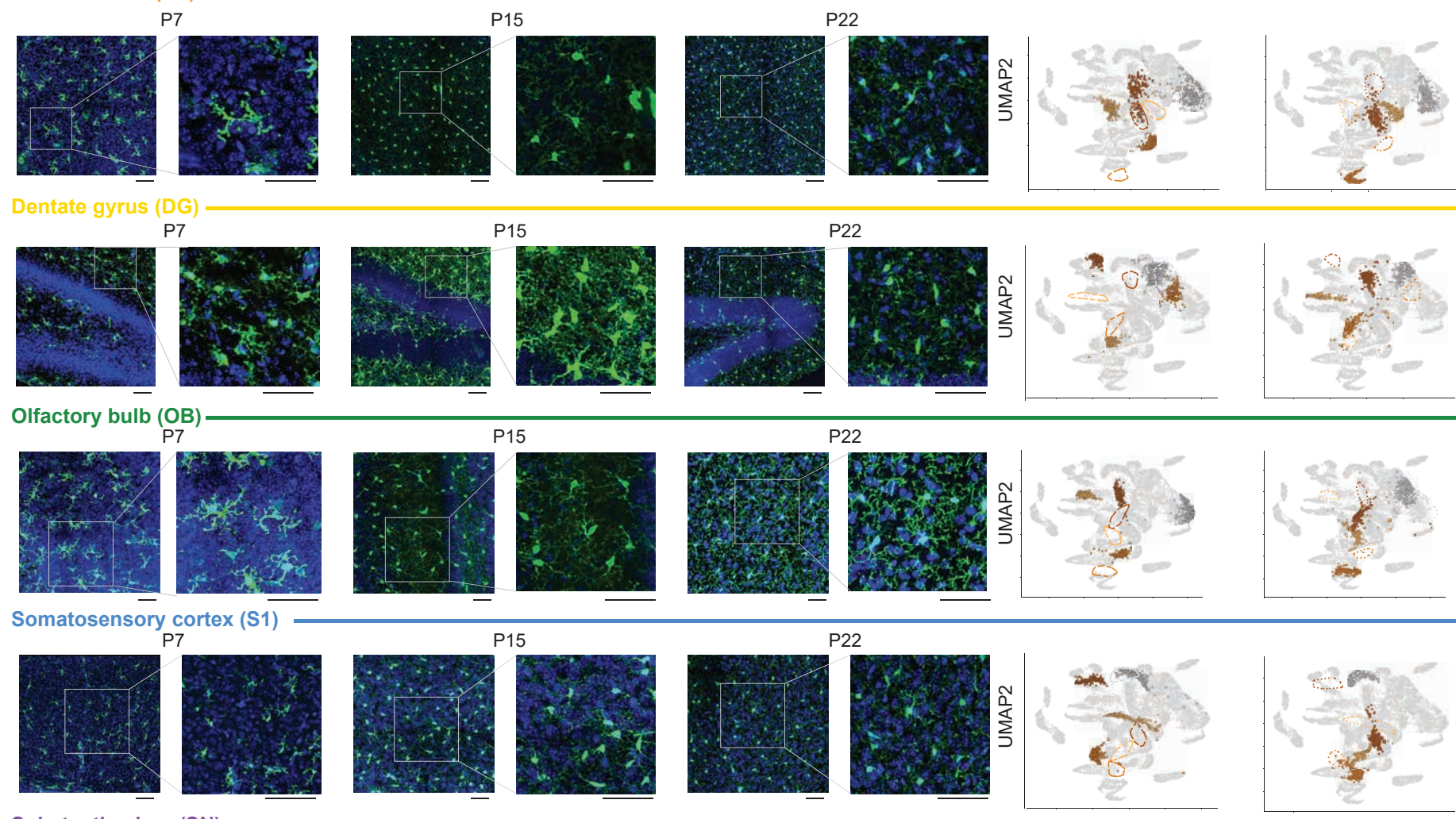

Substantia nigra (SN)
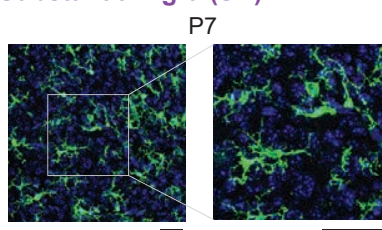

P15
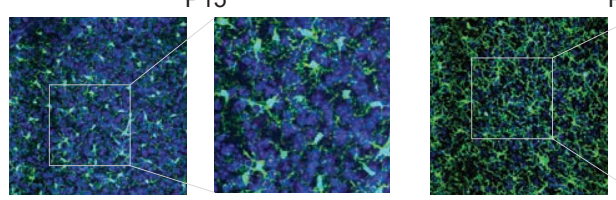

P22
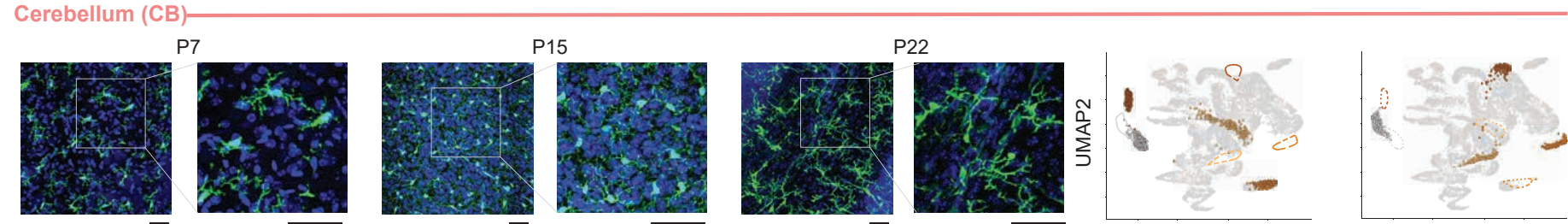

Cochlear nucleus (CN)
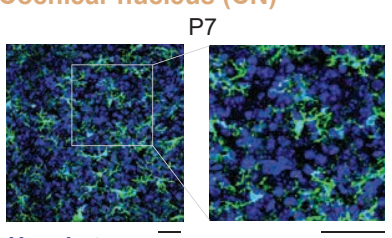

Hoechst
Cx $3 \mathrm{cr} 1^{+/ G F P}$
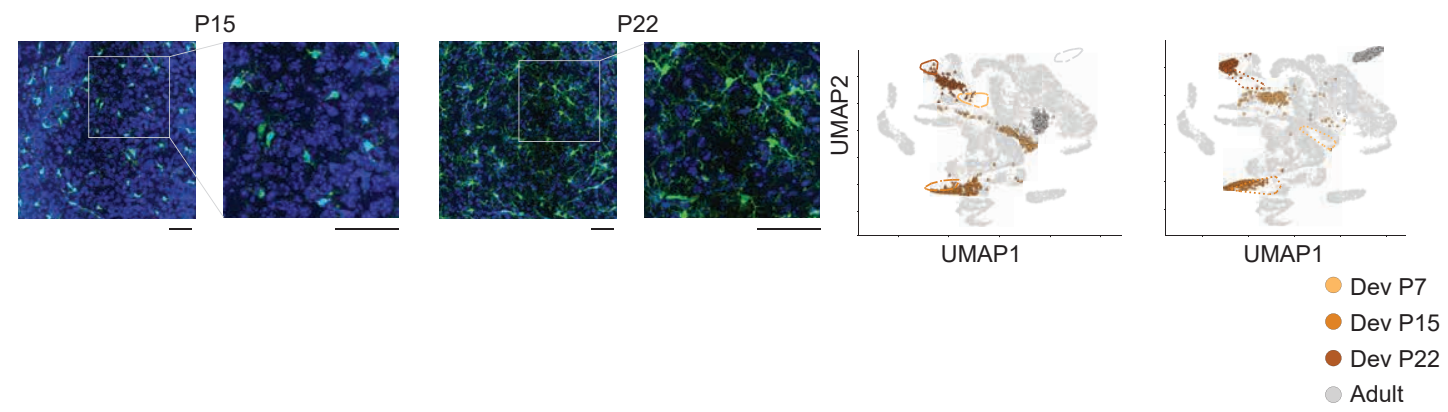


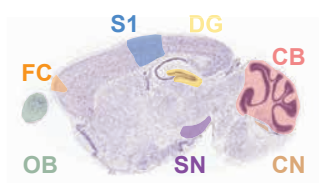

B Microglial morphology during degeneration in 5xFAD

Olfactory bulb (OB)

$5 \times \mathrm{FAD}_{3 \mathrm{~m}}$

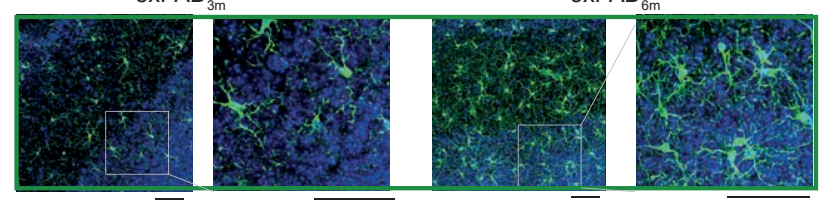

Frontal cortex (FC)

$5 \mathrm{xFAD}$
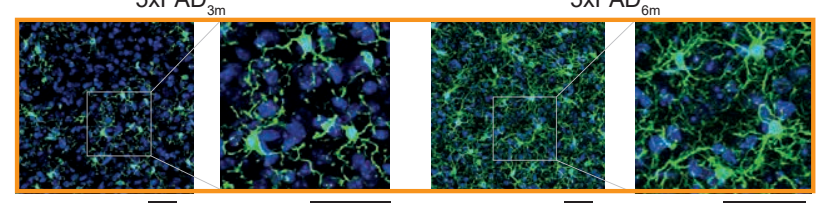

Dentate gyrus (DG)

$5 x F A D_{3 m}$
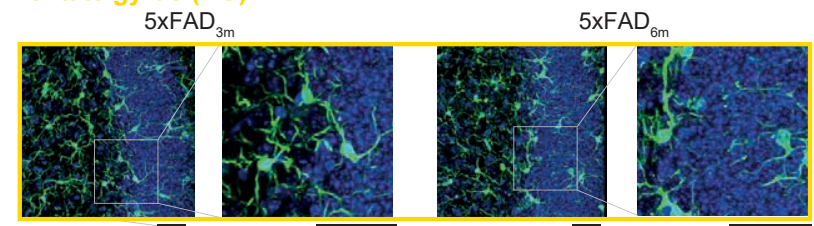

Somatosensory cortex (S1)

$5 \mathrm{xFAD}_{3 \mathrm{~m}}$

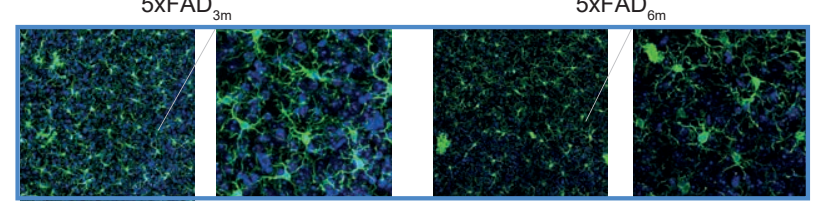

Substantia nigra (SN)

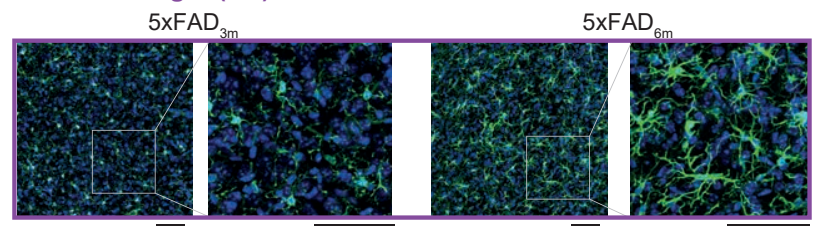

Cerebellum (CB)

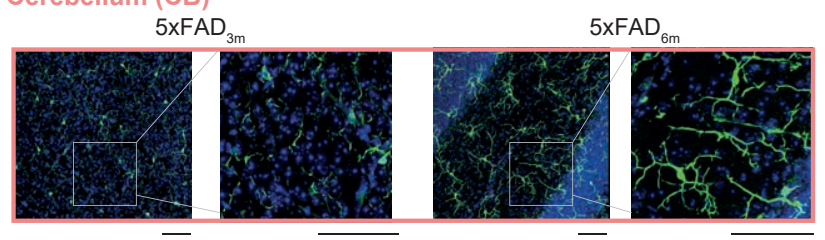

Cochlear nucleus (CN)

$5 \times \mathrm{FAD}_{3 \mathrm{~m}}$
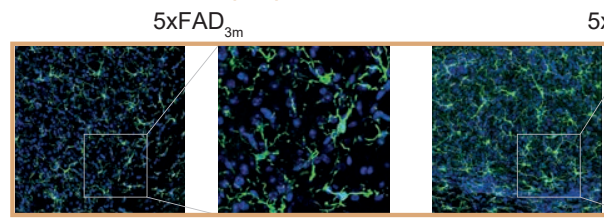

$5 \times F_{6 m}$

Hoechst

lba1

C 5xFAD-Subtraction images of representative bootstrapped persistence images

Control

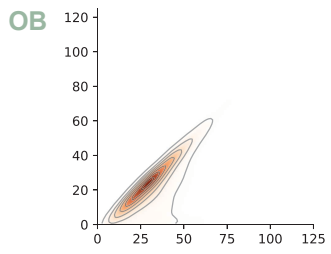

FC
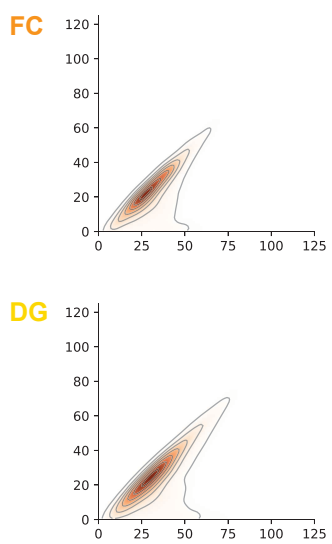

S1

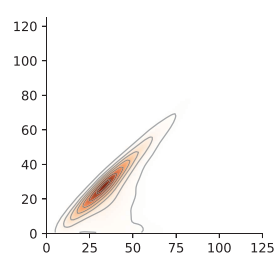

SN

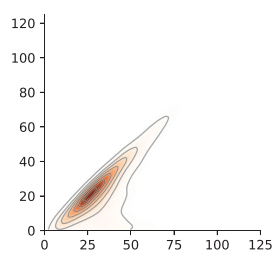

CB

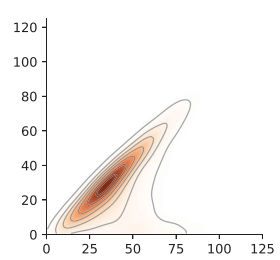

CN

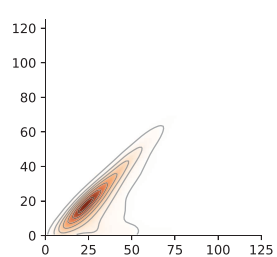

$5 \times F A D$
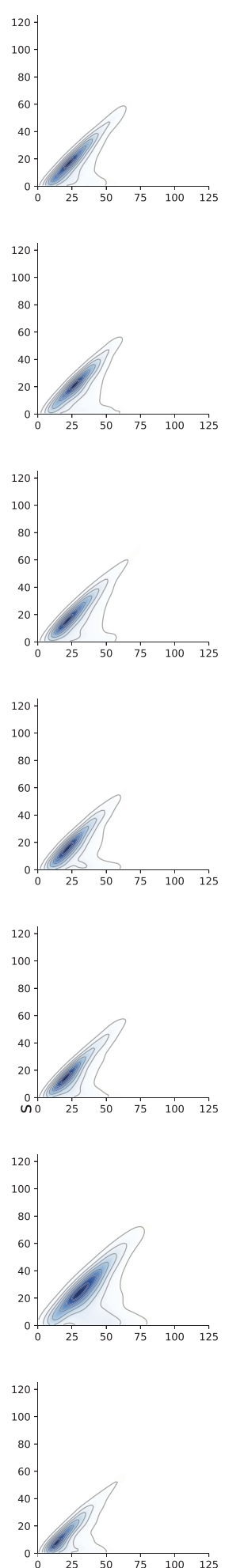
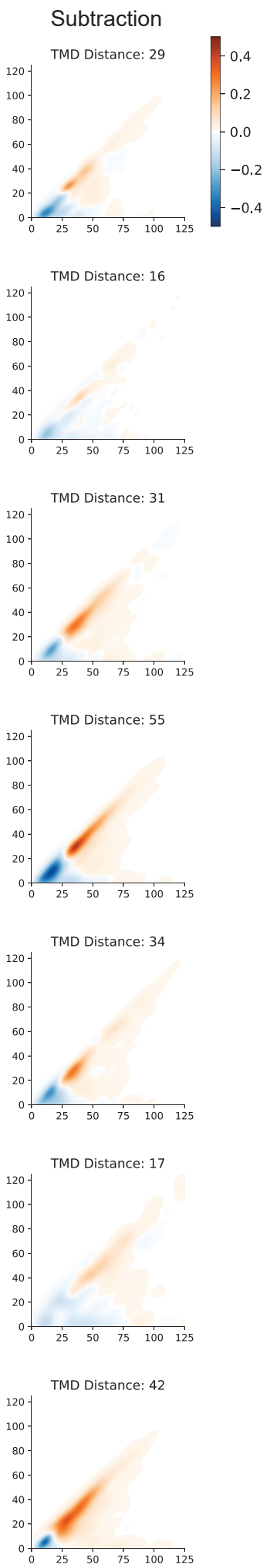


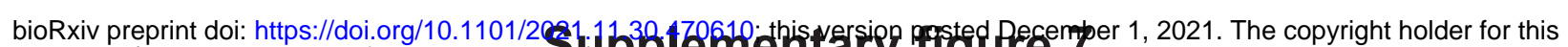

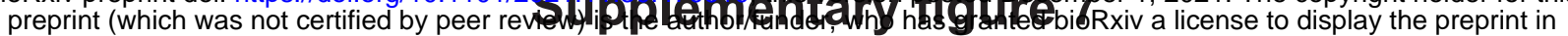
perpetuity. It is made available under aCC-BY-ND 4.0 International license.

A Morphological trajectory of microglia during degeneration in 5xFAD mice

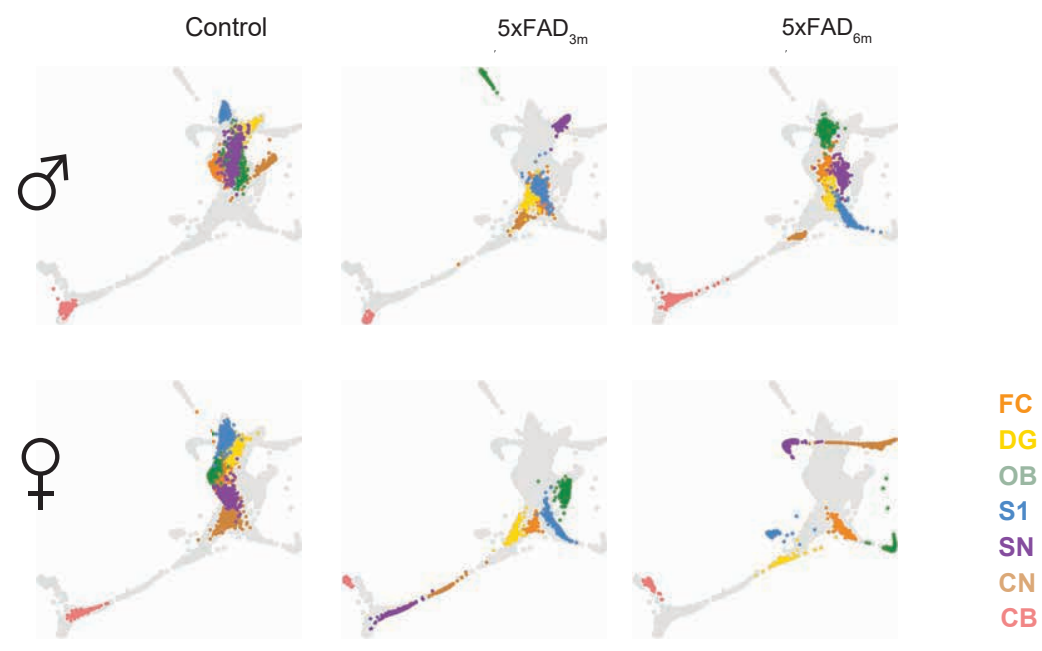

B Sexual dimorphism in morphological trajectory and CD68 in 5xFAD

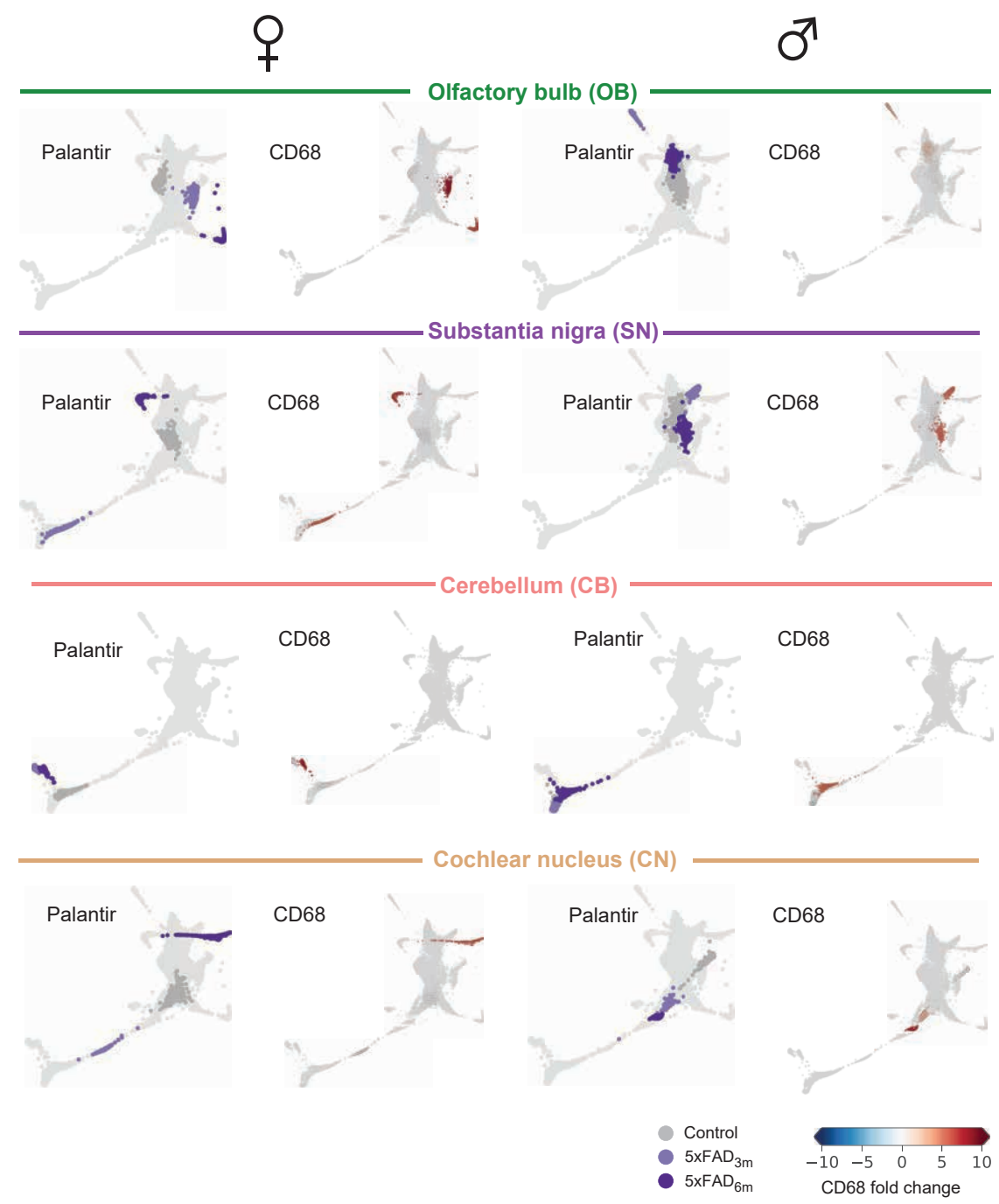


bioRxiv preprint doi: https://doi.org/10.1101/2021.11.30.470610; this version posted December 1, 2021. The copyright holder for this

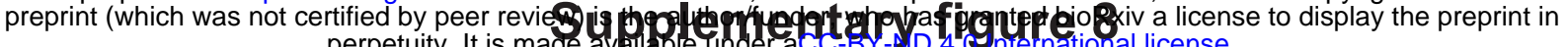

A perpetuity. It is made avellable underact-GY-MD 4.Genternational license.

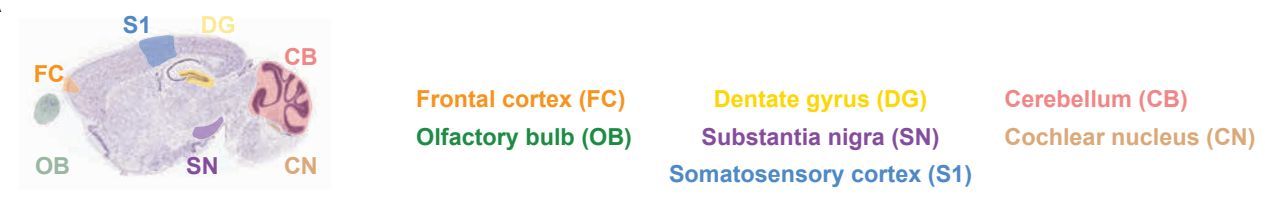

B

Olfactory bulb (OB)

CK-p25 ${ }_{1 \mathrm{w}}$

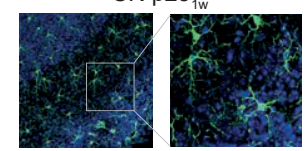

Substantia nigra (SN)

CK-p25

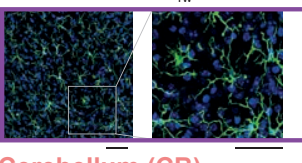

Cerebellum (CB)

CK-p25

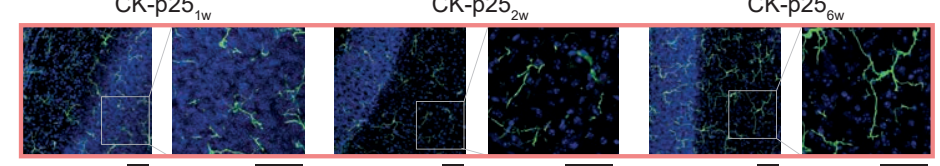

Cochlear nucleus $(\overline{\mathrm{CN}})$

$\mathrm{CK}-\mathrm{p} 25_{1 \mathrm{w}}$

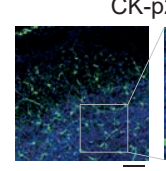

$\mathrm{K}-\mathrm{p} 25_{2 \mathrm{w}}$

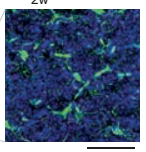

CK-p25

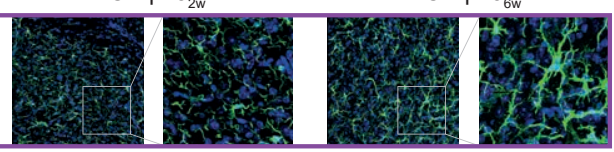

CK-p25 $2 \mathrm{w}$
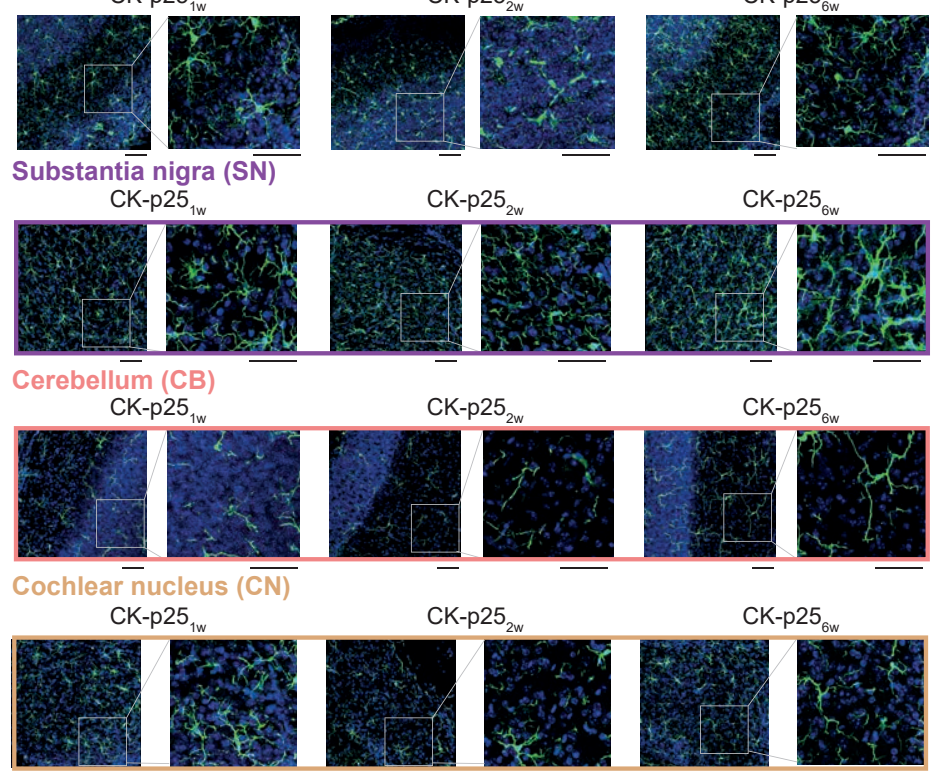

Frontal cortex (FC)

CK-p25

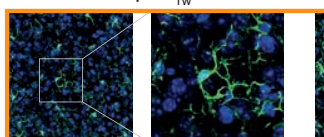

CK-p25 $2 \mathrm{w}$

CK-p25

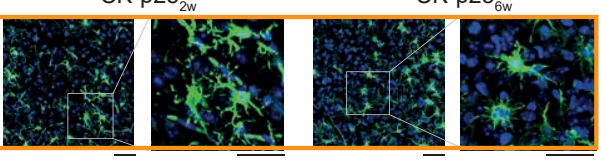

CK-p25

CK-p25 $2 \mathrm{w}$

CK-p25 ${ }_{6 \mathrm{w}}$
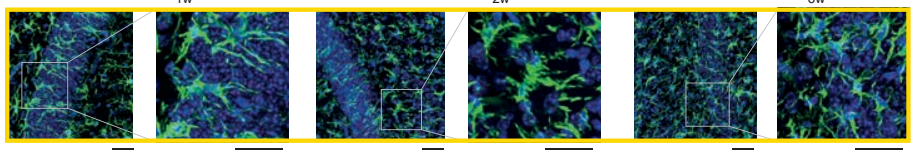

Somatosensory cortex (S1)

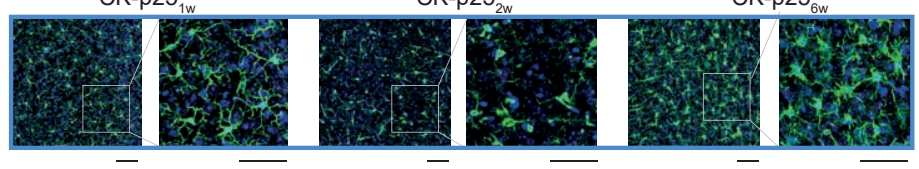

Hoechst

Iba1

C CK-p25-Subtraction images of representative bootstrapped persistence images
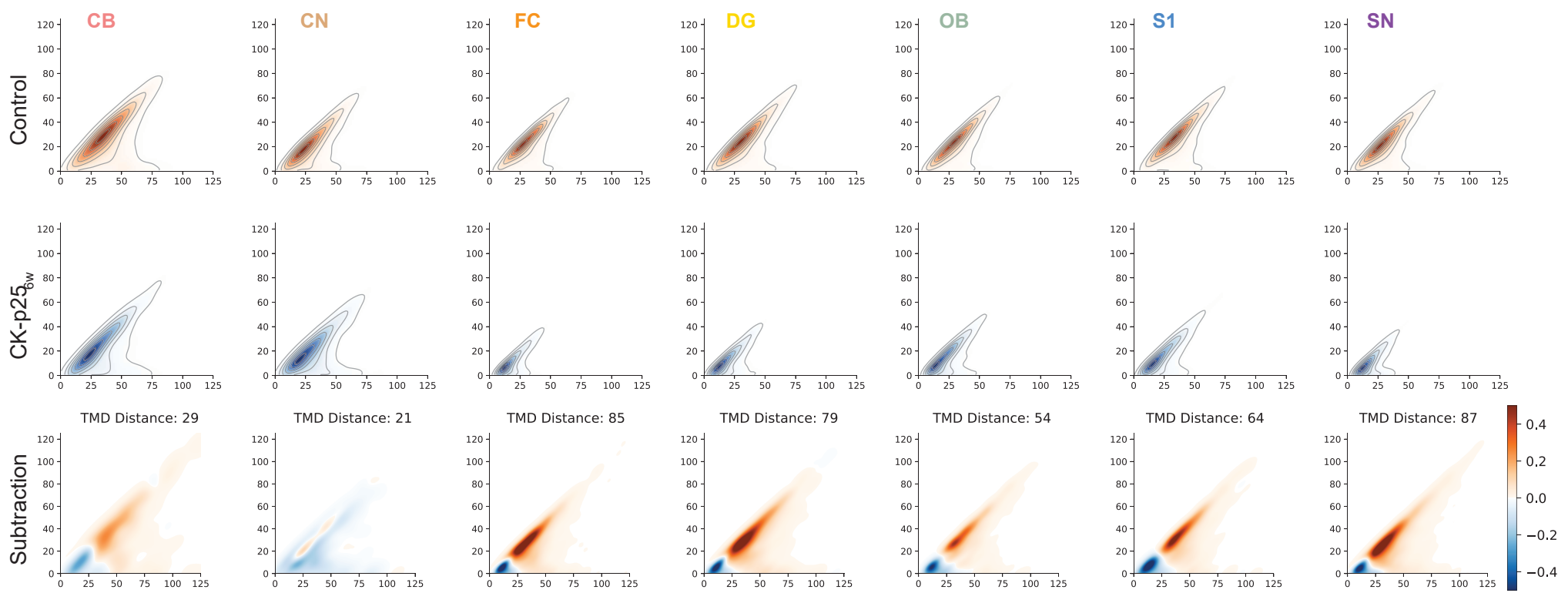
A Morphological trajectory of microglia in CK-p25 mice

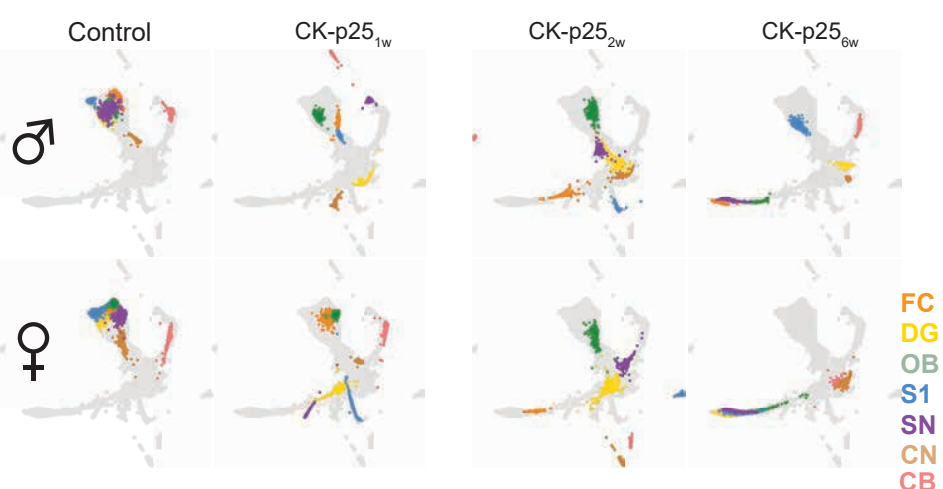

C Sexual dimorphism in morphological trajectory and CD68 in CK-p25

P

$\sigma^{7}$

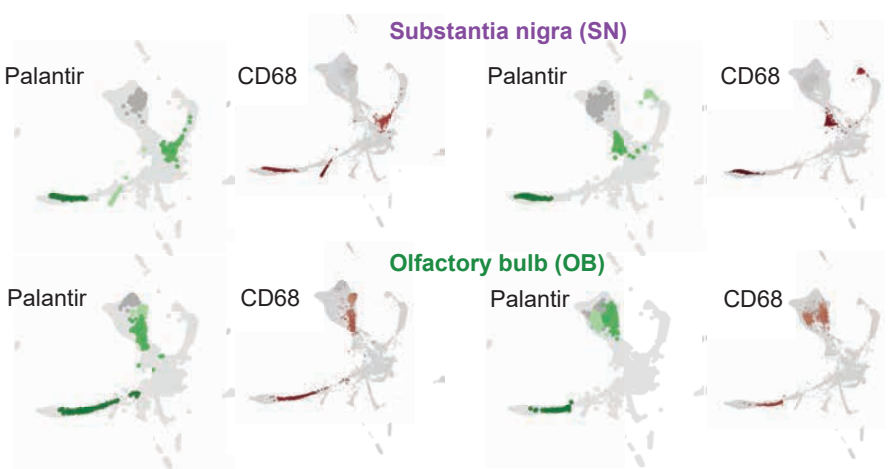

D

q

Cochlear nucleus (CN)

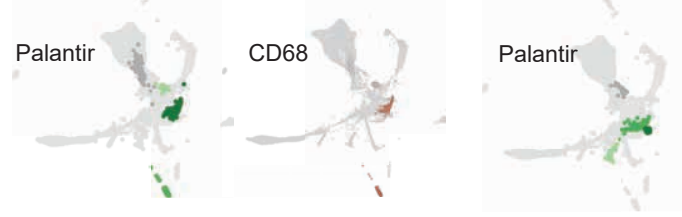

B Sex-specific Monocle trajectory of microglial mophology in CK-p25
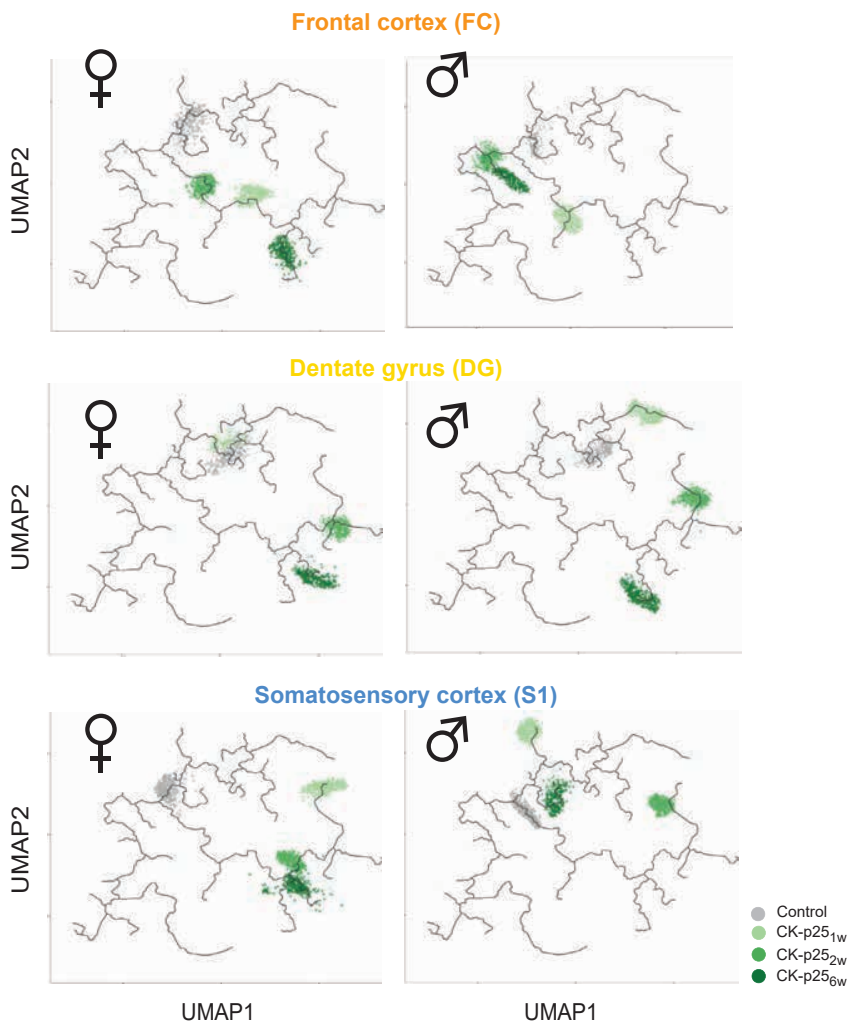

q
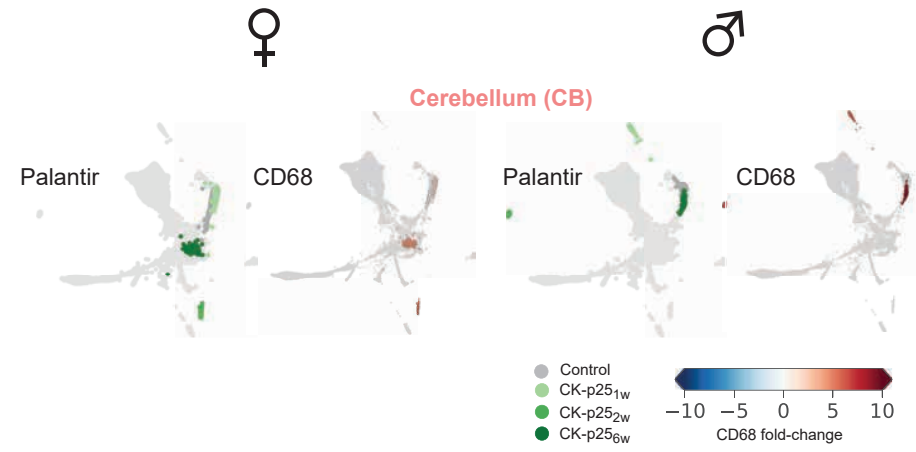


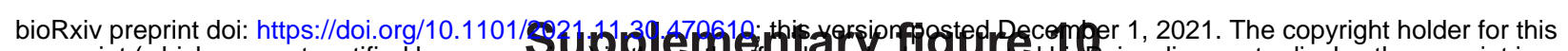

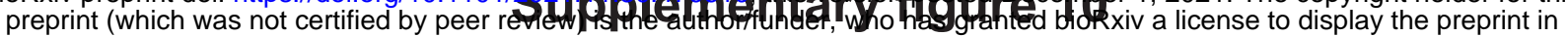
perpetuity. It is made available under aCC-BY-ND 4.0 International license.

A Comparison of female morphological trajectories in development and disease in the most-affected regions

○ Somatosensory cortex

Substantia nigra

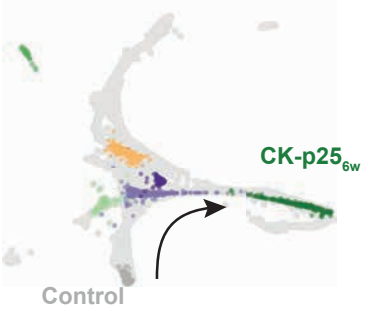

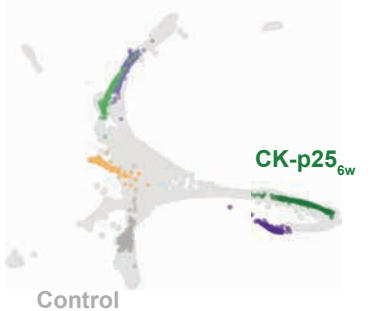

Olfactory bulb

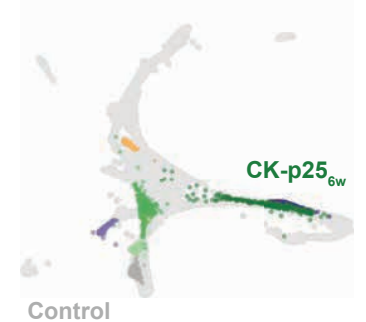

Cochlear nucleus

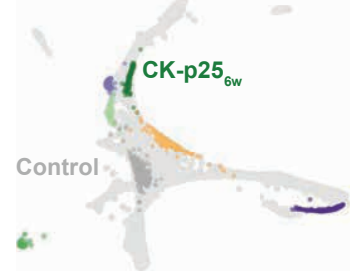

B Comparison of male morphological trajectories in development and disease in the most-affected regions

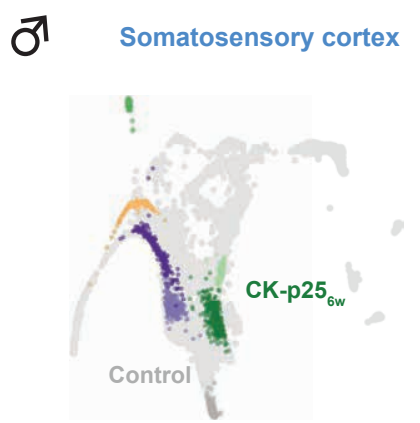

Substantia nigra

Olfactory bulb

Cochlear nucleus
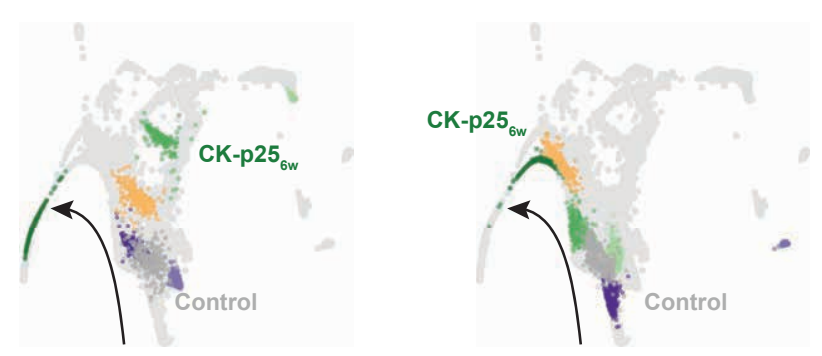

CK-p25

Dev P7

Control

- ${ }_{5 \times F A D} \mathrm{FAD}_{6 \mathrm{~m}}$

-

- CK-p25 $2 \mathrm{w}$

Control 

perpetuity. It is made avalable under aeC-BY $=10$ S 4.0 International license.

A Common morphometric classifiers

Process length $(\mu \mathrm{m})$
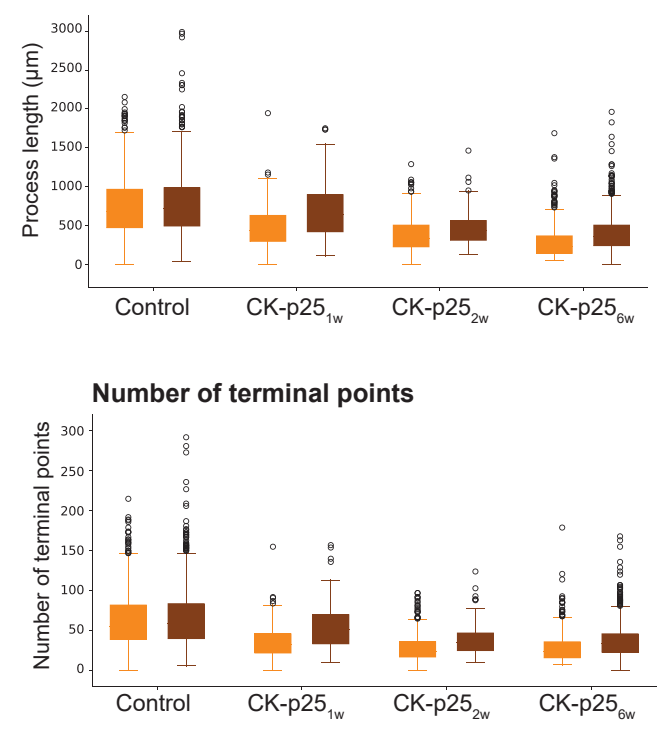

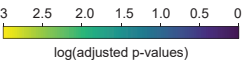

Number of branches

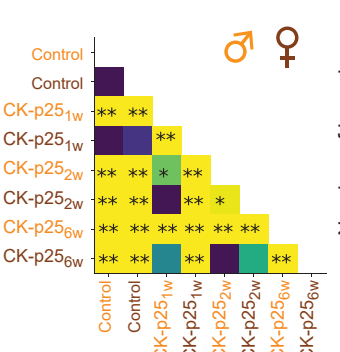

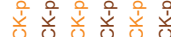
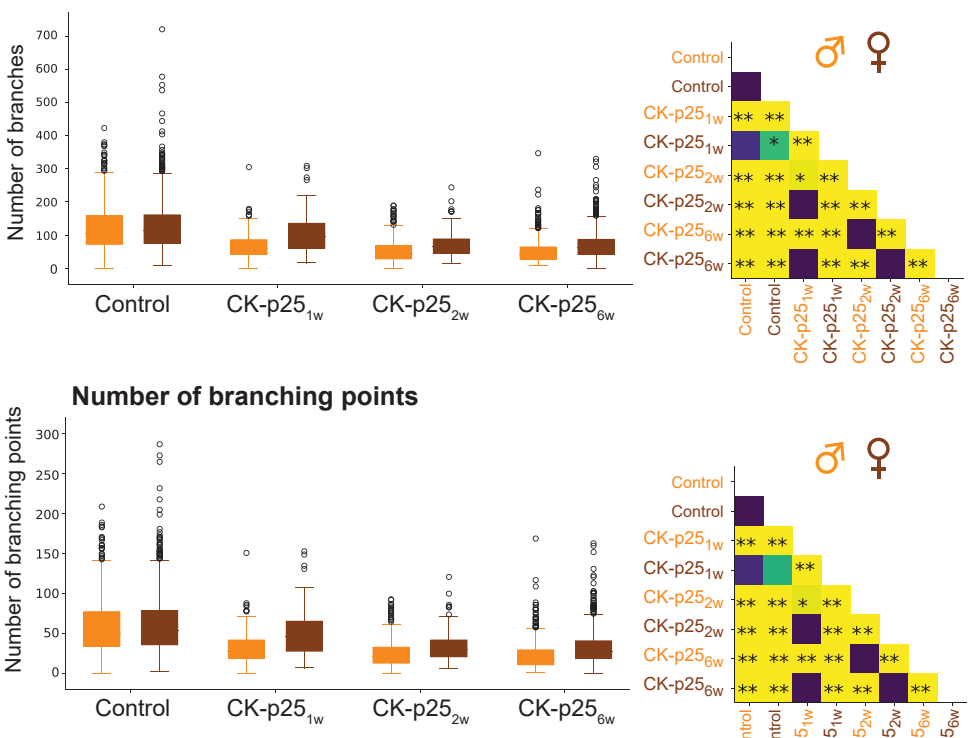

Ђฺ
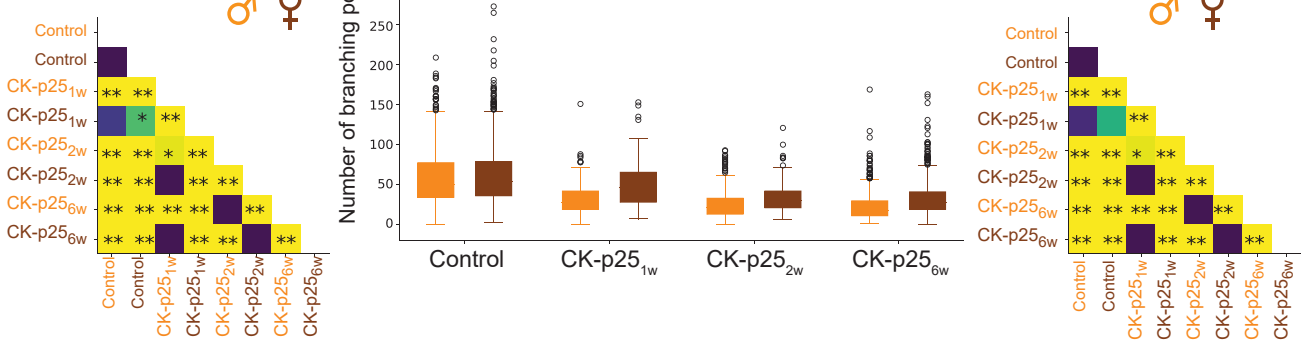

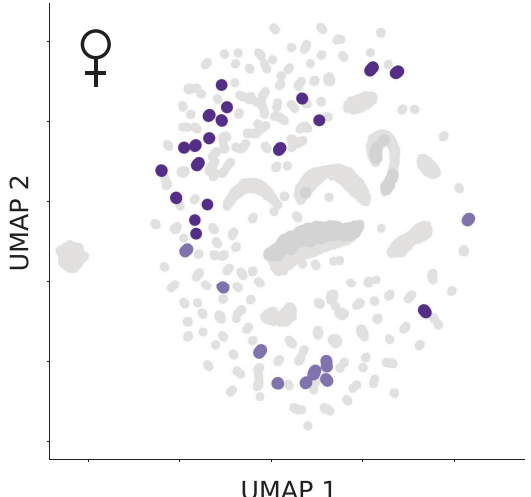

Frontal cortex

C Postnatal development - Bootstrap application to common classifiers

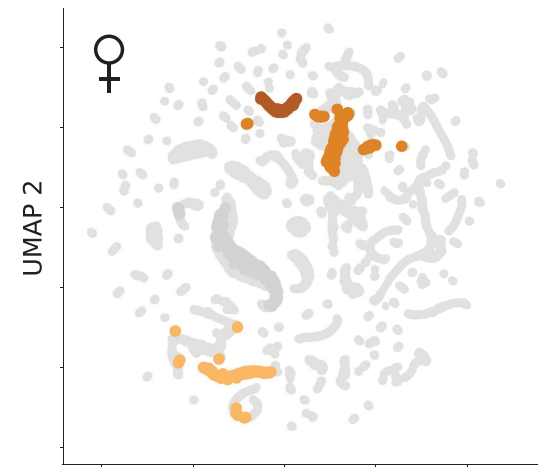

UMAP 1

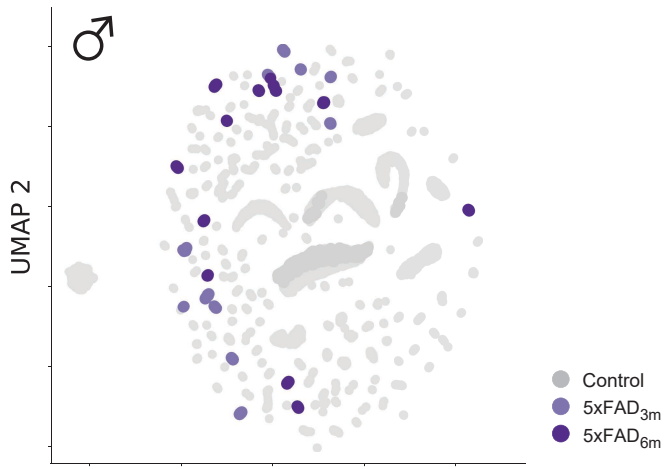

UMAP 1

\section{Frontal cortex}

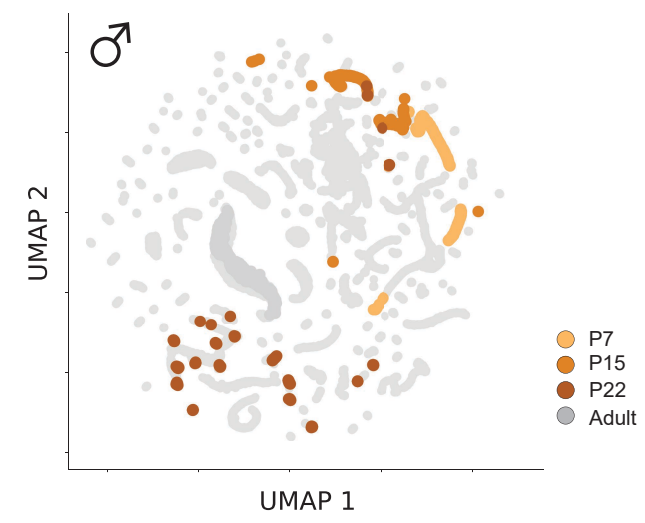




\section{Computational assessment of bootstrapping methods in MorphOMICs}

\section{Single-condition case}

Microglia are highly dynamic (Tremblay et. al., 2011) - a feature which is inherent to their function. Under homeostatic conditions, microglia survey their local environment for insults and anomalies. This intrinsic variability challenges the topological analysis of microglial morphology as we observed in the corresponding persistence images of single microglia (Fig. 1B). This variability can mask heterogeneity between microglial populations from different biological conditions e.g., brain region, sex, and development and disease time point (Supp. Fig. 2B).

To overcome this intrinsic variability within microglial populations, we use bootstrapping methods. Bootstrapping is a statistical method that combines random resampling and permutation. It is commonly used to calculate standard errors, to construct confidence intervals, and to perform hypothesis testing for numerous types of sample statistics. In our case, we used bootstrapping to randomly pool together a pre-determined number of microglia within a condition, called the bootstrap size, to reduce the dispersion. This allowed us to construct a bootstrapped persistence image of this microglial sub-population. By averaging out the highly variable portions of the persistence images, we retain the topological signatures that may separate different conditions. Moreover, bootstrapping makes it possible to create as many bootstrapped persistence images as desired.

The pixels of the persistence image span a high-dimensional space. The bootstrapped persistence images form a point cloud in this high-dimensional space with the average persistence image in the center (Supp. Fig. 2A). Thus, the spread of this cloud allows us to assess the variability within a microglial population. Intuitively, the bootstrap size affects this point cloud size. To construct the bootstrapped persistence images with just a single microglia will give us the largest cloud as it reflects the full size of the population's dispersion. On the other hand, when the bootstrapped persistence image is constructed using all of the microglia, the cloud collapses to a single point as there is no difference between the bootstrapped persistence images and the average persistence image. 
To systematically understand the effect of the bootstrap size on the structure of the point cloud formed by the bootstrapped persistence images, we considered a population composed of a high number of traced microglia, namely the microglia of the adult, healthy dentate gyrus. This allowed us to span a range of sizes for the starting population from which we performed the bootstrapping. First, we only considered microglia traced from male animals to avoid inter-sex differences (Fig. 1F). As we observed no animal-specific batch effects (Supp. Fig. 1C), we selected four mice with the highest number of tracings, and grouped them into two artificial groups (A and B) so that the number of single cells were similar in each group $\left(N_{A}=223, N_{B}=231\right)$. Despite coming from the same brain region, these two groups have a non-zero TMD distance $(d=10.64)$ which we call the TMD intrinsic distance. This intrinsic distance arises due to small but accumulated variations in the persistence images. To account for the effect of unequal starting population sizes between the groups, we randomly selected $n=200$ traced microglia from each group to form the starting population. We then drew single microglia from these groups to create a set of bootstrapped persistence images, which we call bootstrap samples A and B. Note that the TMD intrinsic distance remains the same, regardless of the bootstrap size (Supp. Fig. 2A).

To characterize the point cloud formed by the bootstrapped persistence images, we calculated the within-condition distance which is the average TMD distance between two persistence images within the same bootstrap sample. We want to stress here that the two conditions A and B are artificial: the bootstrap persistence images in bootstrap samples A and B come from the same brain region. We observed that reducing the bootstrap size compacted the point cloud and subsequently reduced the within-condition distance within the bootstrap sample (Supp. Fig. 2C). At a certain bootstrap-to-starting population size ratio where $N_{A}=$ $N_{B}=200$ the within-condition distance becomes smaller than the TMD intrinsic distance. This implies that the between-condition distances, i.e. the TMD distance between persistence images across different bootstrap samples, increases causing a forced separation between two groups from the same condition.

Thus, it is imperative to select a bootstrap size which reduces the dispersion of the bootstrap samples without artificially separating samples that share topological signatures. One way to address the latter condition is to determine whether the bootstrap samples A and B cluster separately under a given bootstrap size. To test this, we performed a complete-linkage 
hierarchical clustering with the TMD distance as the metric. We imposed a cut-off which results in two clusters, $\omega_{1}$ and $\omega_{2}$. We then defined a mixing entropy of the resulting clusters $\Omega=\left\{\omega_{1}, \omega_{2}\right\}$ which measures the discrimination between bootstrap samples and is calculated as

$$
H[\Omega]=\sum_{\omega=\left\{\omega_{1}, \omega_{2}\right\}} H[\omega] \frac{N_{\omega}}{N}
$$

where $H[\omega]=\frac{N_{A}(\omega)}{N_{\omega}} \log _{2} \frac{N_{A}(\omega)}{N_{\omega}}-\left(1-\frac{N_{A}(\omega)}{N_{\omega}}\right) \log _{2}\left(1-\frac{N_{A}(\omega)}{N_{\omega}}\right)$ is the entropy of cluster $\omega$, $N_{A}(\omega)$ is the number of bootstrapped persistence images in bootstrap sample A located in cluster $\omega$ and $N=N_{\omega_{1}}+N_{\omega_{2}}$ is the total number of bootstrapped persistence images in the point cloud. To understand this mixing entropy, we considered both extreme situations. The first case is when bootstrap samples A and B overlap and the point cloud is highly dispersed: this results in a big cluster with all persistence images aggregated except for one. The latter is the last bootstrap persistence image to be clustered in the dendrogram, and the mixing entropy is close to 1 . On the other hand, when the point cloud dispersion was small enough for bootstrap samples A and B to segregate, the clustering resulted in separate clusters for samples A and B, and the mixing entropy is zero. Note, the latter emerges whenever we force the separation between two similar-condition groups.

If we also consider the situation between the two extreme situations, the mixing entropy decreases with increasing bootstrap-to-starting population size ratio (Supp. Fig. 2C). We observed that the mixing entropy remained close to 1 for a range of small ratios and then dropped to zero, depending on the starting population size. This behavior allows us to define an optimal bootstrap size which maximizes the trade-off between the intrinsic variability of the bootstrap samples and the indistinguishability of samples coming from the same conditions. By dividing the mixing entropy with the within-condition distance, we found a peak close to a bootstrap size that is $30 \%$ of the starting population size (Supp. Fig. 2D).

Furthermore, we assessed the effect of low starting population sizes on both the withincondition distances and mixing entropy. Thus, we randomly selected $n=$ $\{10,20,30,40,50,60,70,80\}$ microglia from each group and performed the bootstrapping over the resulting starting populations. We found that both within-condition distance and mixing entropy decreased as a function of the bootstrap size, but dependent on the starting population size (Supp. Fig. 2C). Interestingly, when we divided the mixing entropy by the 
within-condition distance, we observed that there was no longer a well-defined optimal bootstrap size (Supp. Fig. 2D), and that the optimal ratio is a range of parameters which is larger for samples with a low starting population size.

\section{Multiple-condition case: sexual dimorphism in the frontal cortex and dentate gyrus}

In the analyses above, we only considered the situation where two groups come from the same condition, such as microglia from the adult male dentate gyrus. Here, we look at a situation where we have multiple conditions which not only exhibit spatial but also sexual heterogeneity.

We focused on microglial populations coming from the healthy, adult frontal cortex (FC) and the dentate gyrus (DG) where we see a microglial signature and a region-dependent sexual dimorphism. Thus, we took 75 microglia from the four male and four female mice with the highest number of traced microglia in the frontal cortex and dentate gyrus. To investigate the effect of having unequal proportions of male and female microglia in the sample, we created starting populations with size $N=200$ where the male-to-female microglia ratio, $r=$ $\{0.0,0.1,0.2, \ldots, 0.8,0.9,1.0\}$ was fixed. For each brain region and male-to-female ratio, we constructed bootstrap samples with bootstrap-to-starting population size ratio at $0.1,0.3,0.5$, 0.7, and 0.9. As there are multiple conditions, we looked at the 2D UMAP representations for each bootstrap size using the same parameters in the main text.

We observed that at 0.1 size ratio, the difference in the morphological signature between FC and DG microglia is already apparent (Supp. Fig. 2H), and that within a brain region cluster, the pure male and female samples are located at opposite ends. These ends in the $\mathrm{DG}_{\mathrm{mg}}$ cluster tend to come closer together than those of the $\mathrm{FC}_{\mathrm{mg}}$. Note that these observations are captured when the bootstrap-to-starting population size ratio is at 0.3 .

Finally, we observed that as the bootstrap size increased, samples with different male-tofemale size ratios broke apart, suggesting a forced separation between different conditions. However, the rate at which the different conditions became more distinct was not uniform. Indeed, we saw that the male-dominated samples in the $\mathrm{DG}_{\mathrm{mg}}$ still form a cluster at bootstrapto-starting population size ratio 0.9 , which implies that ${ }^{\Uparrow} \mathrm{DG}_{\mathrm{mg}}$ have "stronger" and less variable microglial signatures than their female counterparts. This suggests that spanning a range of size ratios can uncover information on the intrinsic variability. 
bioRxiv preprint doi: https://doi.org/10.1101/2021.11.30.470610; this version posted December $1,2021$. The copyright holder for this

preprint (which was not certified by peer review) is the author/funder, who has granted bioRxiv a license to display the preprint in perpetuity. It is made available under aCC-BY-ND 4.0 International license.

\section{Reference}

1.) Tremblay, M. È., Stevens, B., Sierra, A., Wake, H., Bessis, A., \& Nimmerjahn, A.

(2011). The role of microglia in the healthy brain. Journal of Neuroscience, 31(45), 16064-16069. 


\section{Supplementary Table 1}

\begin{tabular}{|c|c|c|c|c|c|}
\hline & Condition & $\begin{array}{l}\text { Time } \\
\text { point }\end{array}$ & Total & $0^{\pi}$ & 오 \\
\hline \multirow{9}{*}{ 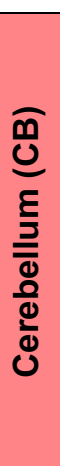 } & Adult & $\begin{array}{l}8-12 \\
\text { weeks }\end{array}$ & 545 & 246 & 299 \\
\hline & \multirow{3}{*}{$\begin{array}{l}\text { Develop- } \\
\text { ment }\end{array}$} & P7 & 399 & 189 & 210 \\
\hline & & P15 & 685 & 175 & 510 \\
\hline & & P22 & 652 & 440 & 212 \\
\hline & \multirow{2}{*}{$5 x F A D$} & 3 months & 109 & 62 & 47 \\
\hline & & 6 months & 138 & 72 & 66 \\
\hline & \multirow{3}{*}{ CK-p25 } & 1 week & 122 & 71 & 51 \\
\hline & & 2 weeks & 65 & 33 & 32 \\
\hline & & 6 weeks & 212 & 97 & 115 \\
\hline
\end{tabular}

\begin{tabular}{|c|c|c|c|c|c|}
\hline \multirow{9}{*}{ 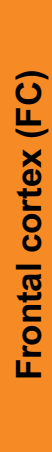 } & Adult & $\begin{array}{l}8-12 \\
\text { weeks }\end{array}$ & 1820 & 894 & 926 \\
\hline & \multirow{3}{*}{$\begin{array}{l}\text { Develop- } \\
\text { ment }\end{array}$} & P7 & 377 & 184 & 193 \\
\hline & & P15 & 953 & 584 & 369 \\
\hline & & P22 & 877 & 407 & 470 \\
\hline & \multirow{2}{*}{$5 x F A D$} & 3 months & 355 & 250 & 105 \\
\hline & & 6 months & 443 & 180 & 263 \\
\hline & \multirow{3}{*}{ CK-p25 } & 1 week & 413 & 194 & 219 \\
\hline & & 2 weeks & 756 & 492 & 264 \\
\hline & & 6 weeks & 1320 & 462 & 858 \\
\hline
\end{tabular}

\begin{tabular}{|c|c|c|c|c|c|}
\hline \multirow{3}{*}{ 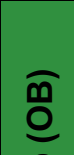 } & Adult & $\begin{array}{l}8-12 \\
\text { weeks }\end{array}$ & 1497 & 701 & 796 \\
\hline & \multirow{3}{*}{$\begin{array}{l}\text { Develop- } \\
\text { ment }\end{array}$} & P7 & 271 & 196 & 75 \\
\hline & & P15 & 477 & 309 & 168 \\
\hline$\overline{\mathbf{z}}$ & & P22 & 698 & 390 & 308 \\
\hline 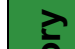 & \multirow{2}{*}{$5 x F A D$} & 3 months & 285 & 82 & 203 \\
\hline & & 6 months & 771 & 244 & 527 \\
\hline & \multirow{3}{*}{ CK-p25 } & 1 week & 284 & 144 & 140 \\
\hline & & 2 weeks & 655 & 223 & 432 \\
\hline & & 6 weeks & 640 & 235 & 405 \\
\hline
\end{tabular}

\begin{tabular}{|c|c|c|c|c|c|}
\hline \multirow{9}{*}{ 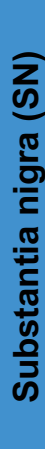 } & Adult & $\begin{array}{l}8-12 \\
\text { weeks }\end{array}$ & 2026 & 976 & 1050 \\
\hline & \multirow{3}{*}{$\begin{array}{l}\text { Develop- } \\
\text { ment }\end{array}$} & P7 & 547 & 350 & 197 \\
\hline & & P15 & 943 & 405 & 538 \\
\hline & & P22 & 644 & 312 & 332 \\
\hline & \multirow{2}{*}{$5 x F A D$} & 3 months & 319 & 166 & 153 \\
\hline & & 6 months & 460 & 202 & 258 \\
\hline & \multirow{3}{*}{ CK-p25 } & 1 week & 264 & 116 & 148 \\
\hline & & 2 weeks & 449 & 249 & 200 \\
\hline & & 6 weeks & 716 & 469 & 247 \\
\hline
\end{tabular}

\begin{tabular}{|c|c|c|c|c|c|}
\hline & Condition & $\begin{array}{l}\text { Time } \\
\text { point }\end{array}$ & Total & $0^{\lambda}$ & 오 \\
\hline \multirow{9}{*}{ 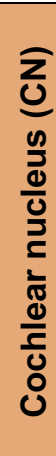 } & Adult & $\begin{array}{l}\text { 8-12 } \\
\text { weeks }\end{array}$ & 754 & 256 & 498 \\
\hline & \multirow{3}{*}{$\begin{array}{l}\text { Develop- } \\
\text { ment }\end{array}$} & $\mathrm{P} 7$ & 477 & 252 & 225 \\
\hline & & P15 & 747 & 413 & 334 \\
\hline & & P22 & 638 & 311 & 327 \\
\hline & \multirow{2}{*}{$5 x F A D$} & 3 months & 236 & 117 & 119 \\
\hline & & 6 months & 170 & 67 & 103 \\
\hline & \multirow{3}{*}{ CK-p25 } & 1 week & 208 & 129 & 79 \\
\hline & & 2 weeks & 248 & 133 & 115 \\
\hline & & 6 weeks & 209 & 72 & 137 \\
\hline
\end{tabular}

\begin{tabular}{|c|c|c|c|c|c|}
\hline \multirow{9}{*}{ 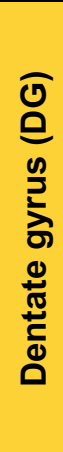 } & Adult & $\begin{array}{l}8-12 \\
\text { weeks }\end{array}$ & 1815 & 913 & 902 \\
\hline & \multirow{3}{*}{$\begin{array}{l}\text { Develop- } \\
\text { ment }\end{array}$} & P7 & 368 & 154 & 214 \\
\hline & & P15 & 439 & 289 & 150 \\
\hline & & P22 & 665 & 286 & 379 \\
\hline & \multirow{2}{*}{$5 x F A D$} & 3 months & 643 & 216 & 427 \\
\hline & & 6 months & 533 & 303 & 230 \\
\hline & \multirow{3}{*}{ CK-p25 } & 1 week & 383 & 223 & 160 \\
\hline & & 2 weeks & 593 & 307 & 286 \\
\hline & & 6 weeks & 1629 & 293 & 1336 \\
\hline
\end{tabular}

\begin{tabular}{|c|c|c|c|c|c|}
\hline 5 & Adult & $\begin{array}{l}8-12 \\
\text { weeks }\end{array}$ & 1540 & 821 & 719 \\
\hline & \multirow{3}{*}{$\begin{array}{l}\text { Develop- } \\
\text { ment }\end{array}$} & P7 & 312 & 165 & 147 \\
\hline & & P15 & 688 & 458 & 230 \\
\hline & & P22 & 786 & 506 & 280 \\
\hline & \multirow{2}{*}{ 5xFAD } & 3 months & 540 & 254 & 286 \\
\hline & & 6 months & 628 & 374 & 254 \\
\hline & \multirow{3}{*}{ CK-p25 } & 1 week & 441 & 177 & 264 \\
\hline & & 2 weeks & 479 & 273 & 206 \\
\hline & & 6 weeks & 834 & 231 & 603 \\
\hline
\end{tabular}




\section{Supplementary Table 2 - related to Supplementary Figure 1A}

\section{Dendritic length}

\begin{tabular}{l|c|c|c|c|c|c|c|}
\multicolumn{2}{c}{ CB } & CN & DG & FC & OB & S1 & SN \\
\hline CB & $1.000 \mathrm{E}+00$ & $1.000 \mathrm{E}+00$ & $3.910 \mathrm{E}-06$ & $8.771 \mathrm{E}-05$ & $2.755 \mathrm{E}-07$ & $4.132 \mathrm{E}-11$ & $1.396 \mathrm{E}-04$ \\
\cline { 2 - 8 } CN & $1.000 \mathrm{E}+00$ & $1.000 \mathrm{E}+00$ & $3.612 \mathrm{E}-04$ & $4.258 \mathrm{E}-03$ & $3.982 \mathrm{E}-05$ & $1.706 \mathrm{E}-08$ & $5.711 \mathrm{E}-03$ \\
DG & $3.910 \mathrm{E}-06$ & $3.612 \mathrm{E}-04$ & $1.000 \mathrm{E}+00$ & $1.000 \mathrm{E}+00$ & $1.000 \mathrm{E}+00$ & $7.192 \mathrm{E}-01$ & $1.000 \mathrm{E}+00$ \\
\hline FC & $8.771 \mathrm{E}-05$ & $4.258 \mathrm{E}-03$ & $1.000 \mathrm{E}+00$ & $1.000 \mathrm{E}+00$ & $1.000 \mathrm{E}+00$ & $2.127 \mathrm{E}-01$ & $1.000 \mathrm{E}+00$ \\
\hline OB & $2.755 \mathrm{E}-07$ & $3.982 \mathrm{E}-05$ & $1.000 \mathrm{E}+00$ & $1.000 \mathrm{E}+00$ & $1.000 \mathrm{E}+00$ & $1.000 \mathrm{E}+00$ & $1.000 \mathrm{E}+00$ \\
\hline S1 & $4.132 \mathrm{E}-11$ & $1.706 \mathrm{E}-08$ & $7.192 \mathrm{E}-01$ & $2.127 \mathrm{E}-01$ & $1.000 \mathrm{E}+00$ & $1.000 \mathrm{E}+00$ & $2.595 \mathrm{E}-01$ \\
\hline SN & $1.396 \mathrm{E}-04$ & $5.711 \mathrm{E}-03$ & $1.000 \mathrm{E}+00$ & $1.000 \mathrm{E}+00$ & $1.000 \mathrm{E}+00$ & $2.595 \mathrm{E}-01$ & $1.000 \mathrm{E}+00$ \\
\hline
\end{tabular}

\section{Number of branches}

\begin{tabular}{c|c|c|c|c|c|c|c|}
\multicolumn{2}{c}{ CB } & CN & DG & FC & OB & S1 & SN \\
\hline CB & $1.000 \mathrm{E}+00$ & $1.000 \mathrm{E}+00$ & $1.533 \mathrm{E}-07$ & $4.747 \mathrm{E}-05$ & $4.124 \mathrm{E}-09$ & $1.330 \mathrm{E}-10$ & $2.383 \mathrm{E}-05$ \\
\cline { 2 - 9 } CN & $1.000 \mathrm{E}+00$ & $1.000 \mathrm{E}+00$ & $2.932 \mathrm{E}-03$ & $1.047 \mathrm{E}-01$ & $2.475 \mathrm{E}-04$ & $1.398 \mathrm{E}-05$ & $5.860 \mathrm{E}-02$ \\
\hline DG & $1.533 \mathrm{E}-07$ & $2.932 \mathrm{E}-03$ & $1.000 \mathrm{E}+00$ & $1.000 \mathrm{E}+00$ & $1.000 \mathrm{E}+00$ & $1.000 \mathrm{E}+00$ & $1.000 \mathrm{E}+00$ \\
\hline FC & $4.747 \mathrm{E}-05$ & $1.047 \mathrm{E}-01$ & $1.000 \mathrm{E}+00$ & $1.000 \mathrm{E}+00$ & $1.000 \mathrm{E}+00$ & $4.746 \mathrm{E}-01$ & $1.000 \mathrm{E}+00$ \\
\hline OB & $4.124 \mathrm{E}-09$ & $2.475 \mathrm{E}-04$ & $1.000 \mathrm{E}+00$ & $1.000 \mathrm{E}+00$ & $1.000 \mathrm{E}+00$ & $1.000 \mathrm{E}+00$ & $1.000 \mathrm{E}+00$ \\
\hline S1 & $1.330 \mathrm{E}-10$ & $1.398 \mathrm{E}-05$ & $1.000 \mathrm{E}+00$ & $4.746 \mathrm{E}-01$ & $1.000 \mathrm{E}+00$ & $1.000 \mathrm{E}+00$ & $9.989 \mathrm{E}-01$ \\
\hline SN & $2.383 \mathrm{E}-05$ & $5.860 \mathrm{E}-02$ & $1.000 \mathrm{E}+00$ & $1.000 \mathrm{E}+00$ & $1.000 \mathrm{E}+00$ & $9.989 \mathrm{E}-01$ & $1.000 \mathrm{E}+00$ \\
\hline
\end{tabular}

\section{Number of branching points}

\begin{tabular}{cccc|c|c|c|c|c|}
\multicolumn{2}{c}{ CB } & CN & DG & FC & OB & S1 & SN \\
\hline CB & $1.000 \mathrm{E}+00$ & $1.000 \mathrm{E}+00$ & $2.334 \mathrm{E}-07$ & $4.446 \mathrm{E}-05$ & $4.897 \mathrm{E}-09$ & $1.197 \mathrm{E}-10$ & $2.250 \mathrm{E}-05$ \\
\cline { 2 - 9 } CN & $1.000 \mathrm{E}+00$ & $1.000 \mathrm{E}+00$ & $4.192 \mathrm{E}-03$ & $1.066 \mathrm{E}-01$ & $3.044 \mathrm{E}-04$ & $1.424 \mathrm{E}-05$ & $6.001 \mathrm{E}-02$ \\
\hline DG & $2.334 \mathrm{E}-07$ & $4.192 \mathrm{E}-03$ & $1.000 \mathrm{E}+00$ & $1.000 \mathrm{E}+00$ & $1.000 \mathrm{E}+00$ & $1.000 \mathrm{E}+00$ & $1.000 \mathrm{E}+00$ \\
\hline FC & $4.446 \mathrm{E}-05$ & $1.066 \mathrm{E}-01$ & $1.000 \mathrm{E}+00$ & $1.000 \mathrm{E}+00$ & $1.000 \mathrm{E}+00$ & $4.721 \mathrm{E}-01$ & $1.000 \mathrm{E}+00$ \\
\hline OB & $4.897 \mathrm{E}-09$ & $3.044 \mathrm{E}-04$ & $1.000 \mathrm{E}+00$ & $1.000 \mathrm{E}+00$ & $1.000 \mathrm{E}+00$ & $1.000 \mathrm{E}+00$ & $1.000 \mathrm{E}+00$ \\
\hline S1 & $1.197 \mathrm{E}-10$ & $1.424 \mathrm{E}-05$ & $1.000 \mathrm{E}+00$ & $4.721 \mathrm{E}-01$ & $1.000 \mathrm{E}+00$ & $1.000 \mathrm{E}+00$ & $9.904 \mathrm{E}-01$ \\
\hline SN & $2.250 \mathrm{E}-05$ & $6.001 \mathrm{E}-02$ & $1.000 \mathrm{E}+00$ & $1.000 \mathrm{E}+00$ & $1.000 \mathrm{E}+00$ & $9.904 \mathrm{E}-01$ & $1.000 \mathrm{E}+00$ \\
\hline
\end{tabular}

Number of terminal points

\begin{tabular}{c|c|c|c|c|c|c|c|}
\multicolumn{2}{c}{ CB } & CN & DG & FC & OB & S1 & SN \\
CB & $1.000 \mathrm{E}+00$ & $1.000 \mathrm{E}+00$ & $9.996 \mathrm{E}-08$ & $4.816 \mathrm{E}-05$ & $3.461 \mathrm{E}-09$ & $1.479 \mathrm{E}-10$ & $2.669 \mathrm{E}-05$ \\
\cline { 2 - 8 } CN & $1.000 \mathrm{E}+00$ & $1.000 \mathrm{E}+00$ & $2.263 \mathrm{E}-03$ & $1.079 \mathrm{E}-01$ & $2.262 \mathrm{E}-04$ & $1.563 \mathrm{E}-05$ & $6.440 \mathrm{E}-02$ \\
\hline DG & $9.996 \mathrm{E}-08$ & $2.263 \mathrm{E}-03$ & $1.000 \mathrm{E}+00$ & $1.000 \mathrm{E}+00$ & $1.000 \mathrm{E}+00$ & $1.000 \mathrm{E}+00$ & $1.000 \mathrm{E}+00$ \\
\hline FC & $4.816 \mathrm{E}-05$ & $1.079 \mathrm{E}-01$ & $1.000 \mathrm{E}+00$ & $1.000 \mathrm{E}+00$ & $1.000 \mathrm{E}+00$ & $4.903 \mathrm{E}-01$ & $1.000 \mathrm{E}+00$ \\
OB & $3.461 \mathrm{E}-09$ & $2.262 \mathrm{E}-04$ & $1.000 \mathrm{E}+00$ & $1.000 \mathrm{E}+00$ & $1.000 \mathrm{E}+00$ & $1.000 \mathrm{E}+00$ & $1.000 \mathrm{E}+00$ \\
S1 & $1.479 \mathrm{E}-10$ & $1.563 \mathrm{E}-05$ & $1.000 \mathrm{E}+00$ & $4.903 \mathrm{E}-01$ & $1.000 \mathrm{E}+00$ & $1.000 \mathrm{E}+00$ & $9.820 \mathrm{E}-01$ \\
\hline SN & $2.669 \mathrm{E}-05$ & $6.440 \mathrm{E}-02$ & $1.000 \mathrm{E}+00$ & $1.000 \mathrm{E}+00$ & $1.000 \mathrm{E}+00$ & $9.820 \mathrm{E}-01$ & $1.000 \mathrm{E}+00$ \\
\hline
\end{tabular}




\section{Supplementary Table 3 - related to Figure 7A}

\section{Dendritic length}

\begin{tabular}{|c|c|c|c|c|c|c|c|c|}
\hline & & \\
\hline & Control & 1 week & 2 weeks & 6 weeks & Control & 1 week & 2 weeks & 6 weeks \\
\hline Control & $1.00 \mathrm{E}+00$ & 1.83E-17 & 1.28E-70 & $3.80 \mathrm{E}-131$ & $1.00 \mathrm{E}+00$ & $1.00 E+00$ & 8.40E-22 & 4.95E-85 \\
\hline 1 week & $1.83 \mathrm{E}-17$ & $1.00 \mathrm{E}+00$ & 5.13E-03 & $1.27 \mathrm{E}-15$ & 7.59E-23 & 1.90E-08 & $1.00 \mathrm{E}+00$ & 4.46E-02 \\
\hline 2 weeks & 1.28E-70 & 5.13E-03 & $1.00 \mathrm{E}+00$ & 2.55E-08 & 6.97E-86 & 4.71E-28 & 1.29E-03 & $1.00 E+00$ \\
\hline 6 weeks & 3.80E-131 & 1.27E-15 & $2.55 \mathrm{E}-08$ & $1.00 E+00$ & $1.40 \mathrm{E}-151$ & $3.74 \mathrm{E}-56$ & 1.34E-18 & $3.16 \mathrm{E}-14$ \\
\hline Control & $1.00 \mathrm{E}+00$ & $7.59 \mathrm{E}-23$ & 6.97E-86 & 1.40E-151 & $E+00$ & 01 & -28 & $4.04 \mathrm{E}-105$ \\
\hline 1 week & $1.00 \mathrm{E}+00$ & 1.90E-08 & 4.71E-28 & $E-56$ & & $1.00 E+00$ & -09 & 8.04E-28 \\
\hline 2 weeks & 8.40E-22 & $1.00 \mathrm{E}+00$ & 1.29E-03 & $E-18$ & 1.57E-28 & 1.01E-09 & $1.00 E+00$ & 1.39E-02 \\
\hline 6 weeks & 4.95E-85 & 4.46E-02 & $1.00 \mathrm{E}+00$ & $3.16 \mathrm{E}-14$ & 4.04E-105 & 8.04E-28 & 1.39E-02 & $1.00 E+00$ \\
\hline
\end{tabular}

\section{Number of branches}

\begin{tabular}{|c|c|c|c|c|c|c|c|c|}
\hline & & & & & & & & \\
\hline & Control & 1 week & 2 weeks & 6 weeks & Control & 1 week & 2 weeks & 6 weeks \\
\hline Control & $1.00 \mathrm{E}+00$ & $1.83 \mathrm{E}-17$ & $1.28 \mathrm{E}-70$ & $3.80 \mathrm{E}-131$ & $1.00 \mathrm{E}+00$ & $1.00 \mathrm{E}+00$ & 8.40E-22 & 4.95E-85 \\
\hline 1 week & $1.83 \mathrm{E}-17$ & $1.00 \mathrm{E}+00$ & $5.13 E-03$ & 1.27E-15 & 7.59E-23 & $1.90 \mathrm{E}-08$ & $1.00 \mathrm{E}+00$ & 4.46E-02 \\
\hline 2 weeks & $1.28 \mathrm{E}-70$ & 5.13E-03 & $1.00 \mathrm{E}+00$ & 2.55E-08 & 6.97E-86 & $4.71 \mathrm{E}-28$ & $1.29 \mathrm{E}-03$ & $1.00 \mathrm{E}+00$ \\
\hline 6 weeks & 3.80E-131 & $1.27 \mathrm{E}-15$ & $2.55 \mathrm{E}-08$ & $1.00 \mathrm{E}+00$ & $1.40 \mathrm{E}-151$ & $3.74 \mathrm{E}-56$ & $1.34 \mathrm{E}-18$ & $3.16 \mathrm{E}-14$ \\
\hline Control & $1.00 \mathrm{E}+00$ & 7.59E-23 & 6.97E-86 & $1.40 \mathrm{E}-151$ & $1.00 \mathrm{E}+00$ & 3.39E-01 & $1.57 \mathrm{E}-28$ & $4.04 \mathrm{E}-105$ \\
\hline 1 week & $1.00 \mathrm{E}+00$ & 1.90E-08 & 4.71E-28 & $3.74 \mathrm{E}-56$ & 3.39E-01 & $1.00 \mathrm{E}+00$ & 1.01E-09 & 8.04E-28 \\
\hline 2 weeks & 8.40E-22 & $1.00 \mathrm{E}+00$ & 1.29E-03 & $1.34 \mathrm{E}-18$ & $1.57 \mathrm{E}-28$ & 1.01E-09 & $1.00 \mathrm{E}+00$ & 1.39E-02 \\
\hline 6 weeks & 4.95E-85 & 4.46E-02 & $1.00 \mathrm{E}+00$ & $3.16 \mathrm{E}-14$ & $4.04 \mathrm{E}-105$ & 8.04E-28 & 1.39E-02 & $1.00 E+00$ \\
\hline
\end{tabular}

\section{Number of branching points}

\begin{tabular}{|c|c|c|c|c|c|c|c|c|c|}
\hline & & \\
\hline & & Control & 1 week & 2 weeks & 6 weeks & Control & 1 week & 2 weeks & 6 weeks \\
\hline \multirow{4}{*}{$0^{\lambda}$} & Control & $1.00 \mathrm{E}+00$ & 9.96E-27 & $3.61 \mathrm{E}-100$ & 1.32E-102 & $1.00 \mathrm{E}+00$ & 3.66E-01 & $7.20 \mathrm{E}-24$ & $1.00 \mathrm{E}-60$ \\
\hline & 1 week & $9.96 \mathrm{E}-27$ & $1.00 E+00$ & 1.31E-03 & 1.80E-04 & 3.13E-32 & $1.71 \mathrm{E}-10$ & $1.00 \mathrm{E}+00$ & $1.00 \mathrm{E}+00$ \\
\hline & 2 weeks & $3.61 \mathrm{E}-100$ & $1.31 \mathrm{E}-03$ & $1.00 \mathrm{E}+00$ & $1.00 \mathrm{E}+00$ & $1.57 \mathrm{E}-115$ & $3.17 \mathrm{E}-34$ & $1.75 \mathrm{E}-08$ & $2.01 \mathrm{E}-11$ \\
\hline & 6 weeks & 1.32E-102 & $1.80 \mathrm{E}-04$ & $1.00 \mathrm{E}+00$ & $1.00 \mathrm{E}+00$ & $8.55 \mathrm{E}-118$ & $2.92 \mathrm{E}-36$ & 8.83E-10 & $2.97 \mathrm{E}-13$ \\
\hline \multirow{4}{*}{ q } & Control & $1.00 \mathrm{E}+00$ & $3.13 E-32$ & $1.57 \mathrm{E}-115$ & $8.55 \mathrm{E}-118$ & $1.00 \mathrm{E}+00$ & 9.41E-03 & 9.93E-30 & 5.78E-75 \\
\hline & 1 week & $3.66 \mathrm{E}-01$ & $1.71 \mathrm{E}-10$ & $3.17 E-34$ & 2.92E-36 & $9.41 \mathrm{E}-03$ & $1.00 E+00$ & $9.21 \mathrm{E}-08$ & $3.02 E-14$ \\
\hline & 2 weeks & 7.20E-24 & $1.00 E+00$ & 1.75E-08 & 8.83E-10 & 9.93E-30 & 9.21E-08 & $1.00 \mathrm{E}+00$ & $1.00 \mathrm{E}+00$ \\
\hline & 6 weeks & 1.00E-60 & $1.00 E+00$ & $2.01 \mathrm{E}-11$ & 2.97E-13 & 5.78E-75 & $3.02 E-14$ & $1.00 \mathrm{E}+00$ & $1.00 \mathrm{E}+00$ \\
\hline
\end{tabular}

Number of terminal points

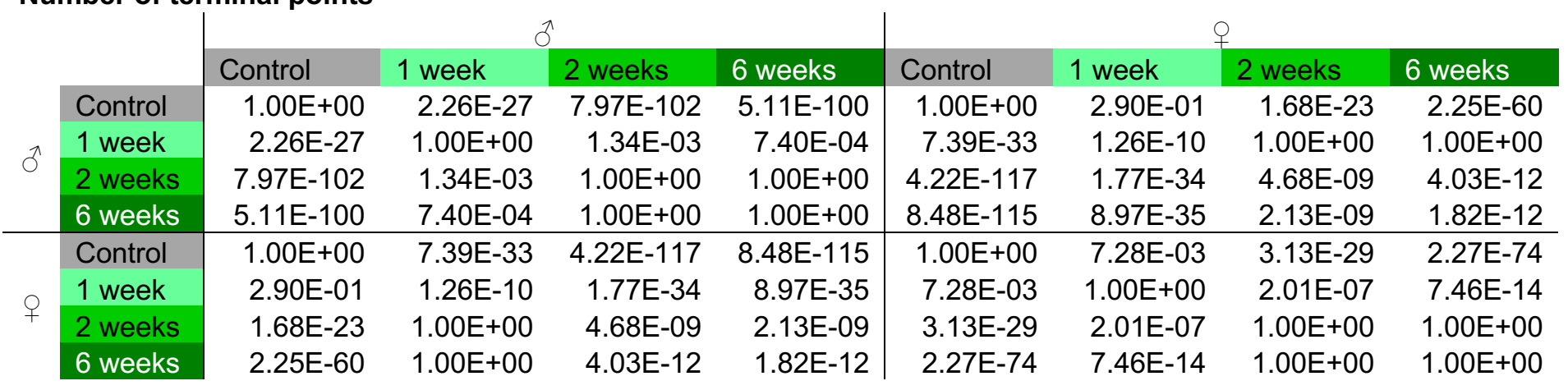




\section{Supplementary Table 4 - \\ related to Figure 7A and Supplementary Fig. 11B-C}

\section{EXTENDED LIST OF MORPHOMETRIC QUANTITIES}

\section{L-measure metric}

\section{Whole tree/microglia size}

Summed total process length

Length

Number of process tips

N_tips

Total_Sum

Total process width

Width

Total_Sum

Total process height

Height

Total_Sum

Total process depth

Depth

Total_Sum

Total_Sum

\section{Bifurcation measures}

Average partition asymmetry

Average local amplitude angle

Maximum local amplitude angle

Average remote amplitude angle

Maximum remote amplitude angle

Average local tilt angle

Maximum local tilt angle

Average remote tilt angle

Maximum remote tilt angle

Average local torque angle

Maximum local torque angle

Average remote torque angle

Maximum remote torque angle

Partition_Asymmetry
Bif_ampl_local
Bif_ampl_local
Bif_ampl_remote
Bif_ampl_remote
Bif_tilt_local
Bif_tilt_local
Bif_tilt_remote
Bif_tilt_remote
Bif_torque_local
Bif_torque_local
Bif_torque_remote
Bif_torque_remote

Average

Average

Maximum

Average

Maximum

Average

Maximum

Average

Maximum

Average

Maximum

Average

Maximum

\section{Process measures}

Average tortuosity

Average fractal dimension

Maximum fractal dimension

Average branch path length

Maximum branch path length

\section{Compartment measures}

Maximum branch order

Average terminal degree

Maximum path distance from soma

Maximum branch helicity

$\begin{array}{ll}\text { Contraction } & \begin{array}{l}\text { Average } \\ \text { Average } \\ \text { Fractal_Dim }\end{array} \\ \text { Fractal_Dim } & \text { Maximum } \\ \text { Branch_pathlength } & \text { Average } \\ \text { Branch_pathlength } & \text { Maximum }\end{array}$

Branch_Order

Terminal_degree

Maximum

PathDistance

Average

Maximum

Helix 